\title{
Jogos markovianos alternados sob incerteza
}

Fábio de Oliveira Franco

\author{
DISSERTAÇÃO APRESENTADA \\ AO \\ Instituto de MATEMÁtica e EstatísticA \\ DA \\ UniversidAde de SÃo PAUlo \\ PARA \\ OBTENÇÃO DO TÍTULO \\ DE \\ Mestre EM CiêNCIAS \\ Programa: Ciência da Computação \\ Orientador: Prof ${ }^{a}$ Dr $^{a}$. Leliane Nunes de Barros
}

Durante o desenvolvimento deste trabalho o autor recebeu auxílio financeiro do $\mathrm{CNPq}$ 


\section{Jogos markovianos alternados sob incerteza}

Esta versão da dissertação contém as correções e alterações sugeridas pela Comissão Julgadora durante a defesa da versão original do trabalho, realizada em 12/11/2012. Uma cópia da versão original está disponível no Instituto de Matemática e Estatística da Universidade de São Paulo.

Comissão Julgadora:

- $\operatorname{Prof}^{\mathrm{a}}$. Dr ${ }^{\mathrm{a}}$. Leliane Nunes de Barros (orientadora) - IME-USP

- Prof $^{\text {a }}$. Dr ${ }^{\mathrm{a}}$. Karina Valdivia Delgado - EACH-USP

- Prof. Dr. Fábio Glagiardi Cozman - EP-USP 


\section{Dedicatória}

À MINHA QUERIDA FAMÍLIA E AO MEU AMOR, VIVIANE. 


\section{Agradecimentos}

Agradeço à professora Leliane pela oportunidade dada, o que com certeza fez com que eu amadurecesse pessoal e profissionalmente. Agradeço à professora Karina e ao professor Fábio por fazerem parte da banca e por terem colaborado diretamente para o desenvolvimento deste trabalho e a tantos outros professores que me ajudaram direta e indiretamente a alcançar essa conquista. Agradeço à minha família pelo apoio dado em todos os momentos, por acreditar em mim, até mais do que eu mesmo. Agradeço também ao meu amor, Viviane, por sempre estar ao meu lado, dando apoio e sendo tão compreensível. Agradeço aos amigos e colegas do Liamf, entre eles, Bustamante, Carlos, Daniel, Erika, Esdras, Fabiano, Filipe, Karina, Luis, Mijail, Paulo de Tarso, Philipe, Ricardo Andrade, Ricardo Augusto, Ricardo Hermann, Ricardo Sekeff, Viviane Meneses, aos "peruanos" e tantos outros que compartilharam ótimos momentos comigo durante minha estadia no IME-USP. E outros amigos que fiz na USP durante o período do mestrado, em especial ao Wiliam, Lisliere, Roger, Dayse e Amanda. Agradeço ao CNPq à bolsa de estudos, a qual foi fundamental para o desenvolvimento deste trabalho. Agradeço aos funcionarios do IME-USP, em especial "às tias do café" pela amizade e momentos de descontração e a tantos outros funcionários sem os quais o IME-USP certamente não seria o mesmo. 


\section{Resumo}

FRANCO, F. O. Jogos markovianos alternados sob incerteza. 2012. 85 f. Dissertação (Mestrado) - Instituto de Matemática e Estatística, Universidade de São Paulo, São Paulo, 2012.

Um Jogo Markoviano Alternado (Alternating Markov Game - AMG) é uma extensão de um Processo de Decisão Markoviano (Markov Decision Process - MDP) para ambientes multiagentes. O modelo AMG é utilizado na tomada de decisão sequencial de $n$ agentes quando são conhecidas as probabilidades de transição das ações a serem tomadas por cada agente. Nesse trabalho estamos interessados em AMGs com probabilidades de transição de estados imprecisas, por exemplo, quando elas são dadas na forma de intervalos de probabilidades. Apresentamos um novo modelo de AMG, que chamamos de Jogo Markoviano Alternado com Probabilidades Imprecisas (Alternate Markov Game with Imprecise Probabilities - AMGIP) que permite que as imprecisões nas probabilidades de transições de estados sejam dadas na forma de parâmetros sujeitos a restrições lineares que estende trabalhos anteriores em que a imprecisão é dada por intervalos de probabilidades (AMG-INTERVAL). Dizemos que a imprecisão representa escolhas da Natureza. A imprecisão desses modelos implica no valor do jogo ser dado por uma função intervalar. Existem diversas formas de calcular a solução do jogo, que depende do comportamento da Natureza e dos critérios de preferência dos jogadores diante das escolhas da Natureza. Assim, neste trabalho discutimos diversas soluções para o AMG-IP e AMG-INTERVAL. Também como resultado do estudo das relações existentes entre os MDPs e os AMGs, propomos um novo modelo chamado de AMG-ST (Alternating Markov Game with Set-valued Transition), capaz de modelar a incerteza do modelo MDP-ST (Markovian Decision Process with Set-valued Transition) como um jogo entre o agente e a Natureza, isto é, um jogo em que a Natureza faz o papel de um dos jogadores.

Palavras-chave: Jogos Markovianos, Probabilidades Imprecisas. 


\begin{abstract}
FRANCO, F. O. Alternating Markov Games under uncertainty. 2012. 85 f. Dissertação (Mestrado) Instituto de Matemática e Estatística, Universidade de São Paulo, São Paulo, 2012.

An Alternating Markov Game (AMG) is an extension of a Markov Decision Process (MDP) for multiagent environments. This model is used on sequencial decision making for $n$ agents when we know the state transition probabilities of actions being taken by each agent. In this work we are interested in AMGs with imprecise probabilities on state transition function, for example, when they are given by probabilities intervals. We present a new AMG model, which we call Alternating Markov Game with Imprecise Probabilities (AMG-IP) that allows imprecision on state transition probabilities given by parameters subject to linear constraints that extend previous works which the imprecision is given by probabilities intervals (AMG-INTERVAL). We say that the imprecision represents the Nature choices. The imprecision of these models implies the game value is given by interval function. There are several ways to calculate the solution of the game, that depend on the behavior of the Nature and the preference criteria of the players on the choices of Nature. Therefore, in this work we discuss various solutions to AMG-IP and AMG-INTERVAL. Also from our study on the relationship among the MDPs and AMGs, we propose a new model called Alternating Markov Game with Set-valued Transition (AMG-ST), that can be used to model the uncertainty of an MDP-ST (Markovian Decision Process with Set-valued Transition) as a result of the match between the agent and the Nature, i.e., a game where the Nature is seen as one of the players.
\end{abstract}

Keywords: Markov Games, Imprecise Probabilities. 


\section{Sumário}

Lista de Abreviaturas $\quad$ xi

$\begin{array}{lll}\text { Lista de Símbolos } & \text { xiii }\end{array}$

$\begin{array}{ll}\text { Lista de Figuras } & \text { xv }\end{array}$

Lista de Tabelas $\quad$ xvii

1 Introdução $\quad 1$

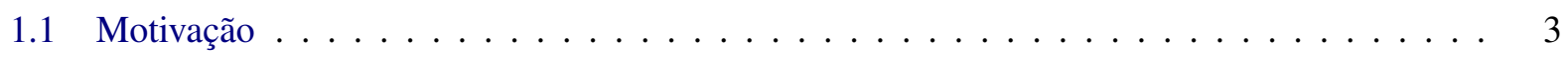

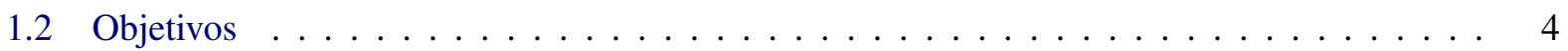

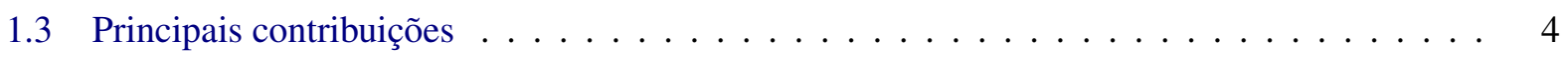

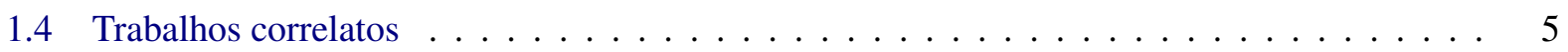

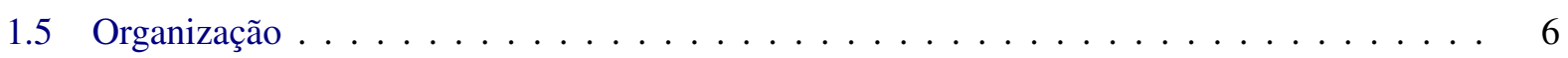

2 Fundamentos $\quad 7$

2.1 Processos de Decisão Markovianos . . . . . . . . . . . . . . . . . . . . . . . 7

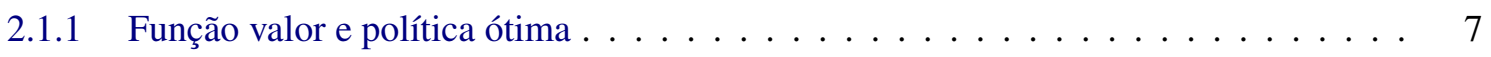

2.1 .2 Prova de convergência $\ldots \ldots \ldots \ldots \ldots \ldots$

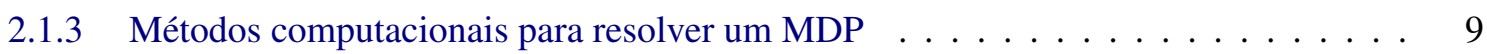

2.2 Processo de Decisão Markoviano com Probabilidades Imprecisas ～. . . . . . . . . . . . . 10

2.2.1 Processo de Decisão Markoviano com Probabilidades Imprecisas dadas por Inter-

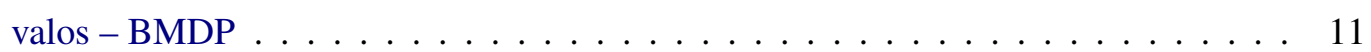

2.2.2 Processo de Decisão Markoviano com Transição Valorada por Conjunto - MDP-ST 12

2.2.3 Processo de Decisão Markoviano com Probabilidades Imprecisas - MDP-IP . . . . . 13

2.2.4 Relações entre BMDPs, MDP-STs e MDP-IPs . . . . . . . . . . . . . . . . . . 15

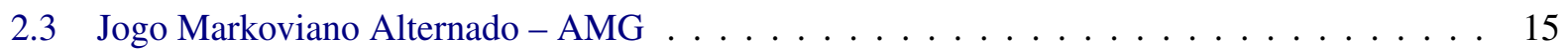

2.3.1 Políticas ótimas de equilíbrio para um AMG . . . . . . . . . . . . . . . . 17

2.3.2 Encontrando pares de políticas ótimas de equilíbrio . . . . . . . . . . . . . . . 17

3 Jogos Markovianos Alternados com Transição Valorada por Conjunto 21

3.1 Um jogo contra a Natureza $\ldots \ldots \ldots \ldots \ldots \ldots \ldots \ldots \ldots \ldots$

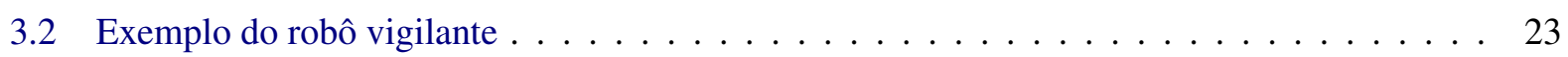

3.3 Jogo Markoviano Alternado com Transição Valorada por Conjunto para o Jogador I - AMG-

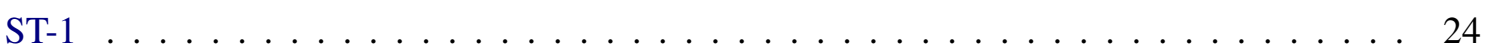


4 Jogos Markovianos Alternados com Probabilidades Imprecisas $\quad 27$

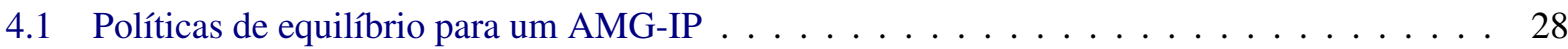

4.1 .1 Função Valor Intervalar . . . . . . . . . . . . . . . . . . . . . . . . . 30

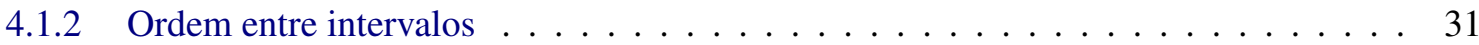

4.2 Diferentes critérios para escolha de pares de políticas de equilíbrio de um AMG-IP . . . . 32

4.2 .1 Critério de preferência Lower-Only . . . . . . . . . . . . . . . . . . 33

4.2 .2 Critério de preferência Upper-Only . . . . . . . . . . . . . . . . . . 33

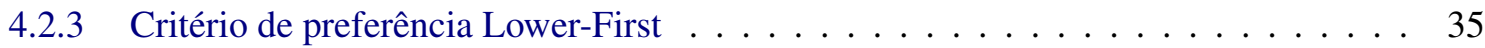

4.2.4 Critério de preferência Upper-First . . . . . . . . . . . . . . . . . . . . . . 39

4.2.5 Comparação entre os critérios Lower-First e Upper-First . . . . . . . . . . . . . . . . 42

4.2.6 Critérios de preferência $\epsilon$-Lower-First e $\epsilon$-Upper-First . . . . . . . . . . . . 42

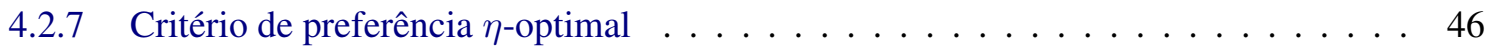

4.2.8 Jogo Markoviano Alternado com Transição Valorada por Conjunto - AMG-ST . . . 48

5 Resultados Experimentais $\quad 51$

5.1 Média da recompensa acumulada esperada $\ldots \ldots \ldots \ldots \ldots \ldots \ldots \ldots$

5.2 Comparação entre as funções valor ótimas calculadas pelos critérios Lower-First e Upper-First 52

5.3 Comparação entre as funções valor ótimas calculadas pelos critérios $\epsilon$-Lower-First e Upper-

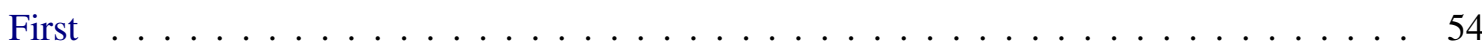

5.4 Comparação entre as funções valor ótimas calculadas pelos critérios $\epsilon$-Upper-First e LowerFirst $\ldots \ldots \ldots \ldots \ldots \ldots \ldots \ldots \ldots \ldots \ldots$

5.5 Comparação entre a média da recompensa acumulada para os critérios Lower-First, UpperFirst, $\epsilon$-Lower-First e $\epsilon$-Upper-First . . . . . . . . . . . . . . . . . . . . . . . 54

5.6 Função valor e média da recompensa acumulada esperada usando o critério $\eta$-Optimal _ . . 56 5.6.1 Comparação entre os tempos de execução dos critérios . . . . . . . . . . . . . . 58

6 Conclusões e Trabalhos Futuros

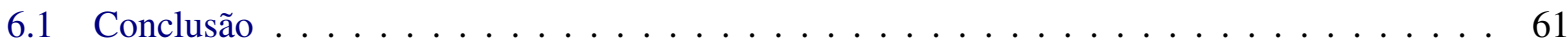

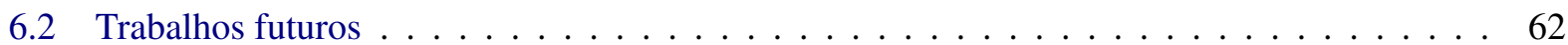

Referências Bibliográficas $\quad 63$ 


\section{Lista de Abreviaturas}

$\begin{array}{ll}\text { AMG } & \text { Alternating Markov Game. } \\ \text { AMG-INTERVAL } & \text { Alternating Markov Game with Imprecise Probabilities given by Intervals. } \\ \text { AMG-IP } & \text { Alternating Markov Game with Imprecise Probabilities. } \\ \text { AMG-ST } & \text { Alternating Markov Game with Set-valued Transition. } \\ \text { AMG-ST-1 } & \text { Alternating Markov Game with Set-valued Transition for player 1. } \\ \text { BMDP } & \text { Bounded-parameter Markov Decision Process. } \\ \text { BRTDP } & \text { Bounded Real Time Dynamic Programming. } \\ \text { LF } & \text { Lower-First. } \\ \text { LRTDP } & \text { Labeled Real Time Dynamic Programming. } \\ \text { MDP } & \text { Markov Decision Process. } \\ \text { MDP-IP } & \text { Markov Decision Process with Imprecise Probabilities. } \\ \text { MDP-ST } & \text { Markov Decision Process with Set-valued Transition. } \\ \text { MG } & \text { Markov Game. } \\ \text { PD } & \text { Probability Distribution. } \\ \text { RTDP } & \text { Real Time Dynamic Programming. } \\ \text { SG } & \text { Stochastic Game. } \\ \text { UF } & \text { Upper-First. }\end{array}$




\section{Lista de Símbolos}

$2^{S} \quad$ Subconjunto de estados de $S$.

$\gamma \quad$ Fator de desconto.

$\pi \quad$ Política do Jogador I.

$\phi \quad$ Política do Jogador II.

$\pi^{*} \quad$ Política ótima do Jogador I.

$\phi^{*} \quad$ Política ótima do Jogador II.

$\eta \quad$ Parâmetro de precaução.

A Conjunto discreto e finito de ações.

$A_{i} \quad$ Conjunto discreto e finito de ações do Jogador $i$.

$A_{1} \quad$ Conjunto discreto e finito de ações do Jogador I.

$A_{2} \quad$ Conjunto discreto e finito de ações do Jogador II.

$K$ Conjunto credal de transição.

$Q \quad$ Função de recompensa esperada.

$R \quad$ Função recompensa.

$R_{i} \quad$ Função recompensa do Jogador $i$.

$R_{1} \quad$ Função recompensa do Jogador I.

$R_{2} \quad$ Função recompensa do Jogador II.

$S \quad$ Conjunto discreto e finito de estados.

$S_{1} \quad$ Conjunto discreto e finito de estados do Jogador I.

$S_{2} \quad$ Conjunto discreto e finito de estados do Jogador II.

$P \quad$ Função de transição probabilística.

$P_{I} \quad$ Função de transição probabilística do Jogador I.

$P_{I I} \quad$ Função de transição probabilística do Jogador II.

$p_{N a t}$ Função de transição probabilística da Natureza.

$V \quad$ Função valor.

$V^{*} \quad$ Função valor ótima. 


\section{Lista de Figuras}

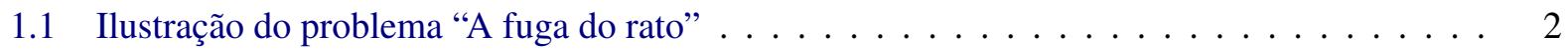

1.2 Função de transição para o problema "A fuga do rato" . . . . . . . . . . . . . . . 2

1.3 Ilustração do problema "O Gato e o Rato" . . . . . . . . . . . . . . . . . . . . 3

2.1 Diagrama de transição de estados para um BMDP . . . . . . . . . . . . . . . . . 11

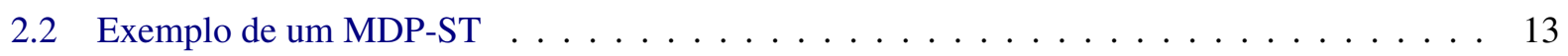

2.3 Duas maneiras de representar a imprecisão nas probabilidades de transição em um MDP. . . 14

2.4 Exemplo de um conjunto credal . . . . . . . . . . . . . . . . . . . 15

2.5 Relacionamentos entre modelos BMDPs, MDP-STs e MDP-IPs . . . . . . . . . . 15

3.1 MDP-ST modelado por um AMG correspondente em que o Jogador II faz o papel da Natureza 22

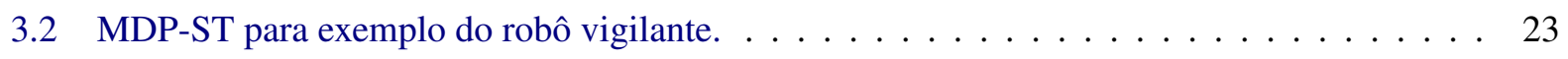

3.3 Um jogo de futebol modelado por um AMG com transições para conjuntos de estados para

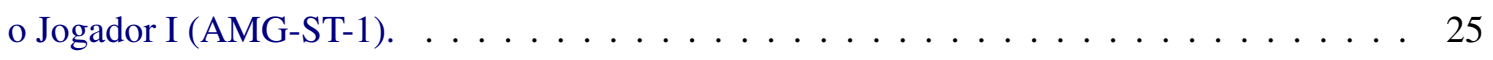

4.1 AMG-IP com probabilidades imprecisas dadas por intervalos e AMG-IP com probabilidades imprecisas dadas por restrições sobre parâmetros . . . . . . . . . . . . . 28

4.2 Os valores ótimos podem não corresponder aos valores de um único par de políticas ótimas. 30

4.3 Comparação entre possíveis valores da função valor geradas pelos pares de políticas ótimas calculadas segundo o critério de preferência Lower-First. . . . . . . . . . . . . 36

4.4 Comparação entre possíveis intervalos do tipo contidos da função valor. Usando a Definição 4.9 de pares de políticas de equilíbrio de Chang (2006), ambos os pares, $\left\langle\pi^{*}, \phi^{*}\right\rangle$ e $\left\langle\pi^{\prime}, \phi^{*}\right\rangle$, são pares de políticas de equilíbrio, enquanto que usando a Definição 4.8 , somente o par $\left\langle\pi^{\prime}, \phi^{*}\right\rangle$ é considerado um par de políticas de equilíbrio. . . . . . . . . . . . . . 42

4.5 Diferentes possibilidades de configuração da função valor intervalar para os critérios LowerFirst (LF) e Upper-First (UF) . . . . . . . . . . . . . . . . . . . . . . . 43

4.6 Valores das funções valor geradas pelos pares de políticas $\left\langle\pi_{1}, \phi_{1}\right\rangle$ e $\left\langle\pi_{2}, \phi_{1}\right\rangle$. . . . . . 44

4.7 Valores das funções valor geradas pelos pares de políticas $\left\langle\pi_{1}, \phi_{1}\right\rangle$ e $\left\langle\pi_{2}, \phi_{1}\right\rangle$. . . . . . . 44

4.8 Valores das funções valor geradas pelos pares de políticas $\left\langle\pi_{1}, \phi_{1}\right\rangle$ e $\left\langle\pi_{2}, \phi_{1}\right\rangle \ldots \ldots$. . . . 45

4.9 Comparação entre possíveis valores da função valor gerados pelos pares de políticas $\langle\pi, \phi\rangle$ $\mathrm{e}\left\langle\pi^{\prime}, \phi^{\prime}\right\rangle$ para $\eta=0.5 \ldots \ldots \ldots \ldots \ldots \ldots \ldots \ldots$

4.10 Diferentes escolhas para o parâmetro de precaução. O valor de mudança de fase $\eta_{f}$ faz com que para valores $\eta>\eta_{f}$, o intervalo $\left[p_{1}, q_{1}\right]$ seja considerado maior que $\left[p_{2}, q_{2}\right]$; para valores $\eta<\eta_{f}$, o intervalo $\left[p_{2}, q_{2}\right]$ é considerado o maior; e com $\eta=\eta_{f}$ os intervalos são

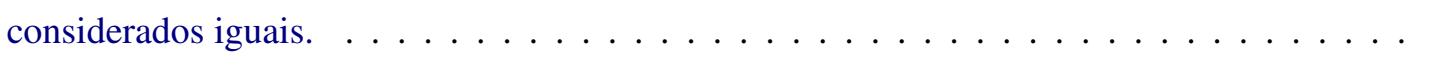


4.11 Intervalos do tipo "contidos". O eixo $x$ é usado para dimensionarmos as medidas de $p_{1}, p_{2}$, $q_{1}$ e $q_{2}$ com relação à origem, e as distâncias $d_{1}$ e $d_{2}$. Assumimos que exista um ponto $x \mathrm{e}$ queremos encontrar $\eta_{f}$ tal que as restrições ao lado sejam satisfeitas.

4.12 Exemplo de um AMG-ST. O par de ações $\left\langle a_{1}\right.$, noop $\rangle$ aplicado no estado $s_{1}$ levam os jogadores para o conjunto $\left\{s_{2}, s_{4}\right\}$ com probabilidade 0.3 e para o conjunto $\left\{s_{4}, s_{6}\right\}$ com probabilidade $0.7 \ldots \ldots \ldots \ldots \ldots \ldots \ldots \ldots \ldots$

5.1 Ilustração do exemplo usado nos experimentos. . . . . . . . . . . . . . . . . 53

5.2 Valores intervalares da função valor alcançados pelas políticas ótimas segundo os critérios de preferência Lower-First e Upper-First para o jogo do Gato e o Rato. O número total de estados para a instância 7x7 é de 4416. O gráfico mostra apenas os estados de 2484 a 2500. Note que para alguns estados as soluções coincidem enquanto para outros as soluções são diferentes indicam intervalos contidos. . . . . . . . . . . . . . . . 5

5.3 Função valor para os estados do Jogador I, seguindo as políticas ótimas geradas pelos critérios de preferência $\epsilon$-Lower-First e $\epsilon$-Upper-First para o jogo do Gato e o Rato. . . . . . . . . 54

5.4 Valores intervalares da função valor alcançados pelas políticas ótimas segundo os critérios de preferência $\epsilon$-Lower-First e $\epsilon$-Upper-First para o jogo do Gato e o Rato. . . . . . . . . . . 55

5.5 Média da recompensa acumulada esperada, no estado $s_{0}$, obtida pelos critérios Lower-First e Upper-First, simulando uma Natureza aleatória. . . . . . . . . . . . . . . . . . . . . . . 55

5.6 Média da recompensa acumulada esperada, no estado $s_{0}$, obtida pelos critérios $\epsilon$-LowerFirst e $\epsilon$-Upper-First simulando uma Natureza aleatória. . . . . . . . . . . . . . . . . . . 56

5.7 Média da recompensa esperada acumulada pelas políticas ótimas encontradas pelos critérios de preferência Lower-First, Upper-First, $\epsilon$-Lower-First e $\epsilon$-Upper-First para o jogo do Gato e o Rato. . . . . . . . . . . . . . . . . . . . . . . . . . . . . .

5.8 Função valor das políticas ótimas devolvidas pelos critérios de preferência Lower-Only, Upper-Only e $\eta$-Optimal para valores de $\eta=\{0.25,0.50,0.75\}$ na instância $3 \times 3$ do jogo do Gato e o Rato. . . . . . . . . . . . . . . . . . . . . . . . . . . . . 57

5.9 Média da recompensa acumulada esperada obtida pelo critério $\eta$-Optimal em que $\eta$ é 0.25 , 0.5 ou 0.75 , simulando uma Natureza aleatória. . . . . . . . . . . . . . . . . . . . 58

5.10 Média da recompensa acumulada esperada obtida pelos critérios $\epsilon$-Lower-First, $\epsilon$-UpperFirst e $\eta$-Optimal, em que $\eta=0.75$, simulando uma Natureza aleatória, para instâncias $n \times n$ com $n$ variando de 3 a $8 \ldots \ldots \ldots \ldots \ldots \ldots \ldots$

5.11 Tempo gasto pelos critérios Lower-First, Upper-First, $\epsilon$-Lower-First, $\epsilon$-Upper-First e $\eta$ Optimal, em que $\eta=0.75$ para instâncias $n \times n \operatorname{com} n$ variando de 3 a 8 . 


\section{Lista de Tabelas}

3.1 Comparação entre o cálculo da função valor para o problema do robô vigilante modelado como um MDP-ST e como um AMG . . . . . . . . . . . . . . . . . . . . . . . . 24

4.1 Conjunto completo de relação entre dois intervalos (R1, R2, R3 e R4) e as diferentes definições de ordenação entre eles. Os intervalos destacados são os maiores de acordo com a definição de ordem entre intervalos escolhida. . . . . . . . . . . . . . . . . . . . 32 


\section{Capítulo 1}

\section{Introdução}

Planejamento é a sub-área da inteligência artificial que estuda como um agente deve realizar tarefas por meio da escolha de ações sequenciais (Russell e Norvig 2010). A solução para um problema de planejamento é, portanto, uma sequência de ações que, ao ser executada, leva o agente do estado inicial para o estado meta.

No planejamento clássico o agente tem conhecimento do mundo em que está situado, das ações que podem ser realizadas e dos seus resultados. Mas há situações em que a tomada de decisão de um agente deve ser realizada mesmo sem o conhecimento preciso sobre os resultados das ações. A essa abordagem dá-se o nome de Planejamento sob Incerteza (Russell e Norvig 2010).

Várias técnicas vêm sendo utilizadas para resolver problemas de planejamento envolvendo incerteza (Bonet e Geffner 2003; Boutilier et al, 1999; Delgado 2010; Guestrin 2003; McMahan et al, 2005; Trevizan , 2006). Um modelo que se destaca é o Processo de Decisão Markoviano (Markov Decision Process MDP), em que as ações possuem efeitos probabilísticos. O processo é dito ser de Markov quando é feita a suposição de Markov, ou seja, o efeito de uma ação em um estado depende apenas da ação e do estado atual do sistema (Puterman 1994). O Exemplo 1.1 ilustra como podemos descrever um problema de planejamento sob incerteza usando MDPs.

Exemplo 1.1 (Domínio Fuga do Rato ${ }^{1}$ ). Um agente (representado por um rato), está situado num ambiente representado por uma grade $3 \times 4$ mostrado na Figura 1.1(a) contendo um obstáculo, uma entrada para um buraco e uma ratoeira. Todo o ambiente é cercado por paredes. O objetivo do rato é encontrar o melhor caminho para chegar ao buraco sem cair na ratoeira. O ambiente foi modelado de forma que os estados são caracterizados pela posição do rato (o buraco, a ratoeira e o obstáculo são fixos para cada problema). No estado inicial, ilustrado na Figura 1.1( a), o rato está na posição $(3,1)$, a ratoeira está na posição $(2,4) e$ o buraco está na posição $(1,4)$. Note que assumimos que o ambiente é completamente observável e estático, ou seja, o rato sempre sabe onde ele está e sabe onde estão o buraco e a ratoeira. Assumimos que o tempo é discretizado e que todas as ações são instantâneas. A interação com o ambiente termina quando o rato alcança um dos estados finais, o buraco ou a ratoeira, e que têm recompensas +1 e -1 , respectivamente. Em cada posição, as ações disponíveis são: mover_para_Norte, mover_para_Sul, mover_para_Oeste e mover_para_Leste. Nas demais posições a recompensa é zero.

Suponha que no Exemplo 1.1 o rato, por algum motivo, esteja um pouco desorientado ou que o chão esteja escorregadio. Isso faz com que as ações escolhidas pelo rato nem sempre tenham o resultado esperado. Se o ambiente fosse determinístico, seria fácil encontrar uma solução: [mover_para_Norte, mover_para_Norte, mover_para_Leste, mover_para_Leste, mover_para_Leste]. Infelizmente, o ambiente nem sempre aceitará essa solução, porque as ações são pouco confiáveis. O modelo específico de movimento estocástico que adotamos está ilustrado na Figura 1.1(b). Cada ação alcança o efeito pretendido com probabilidade 0.8 e 0.1 para sua direita ou esquerda. Além disso, se o rato bater em uma parede, ele permanecerá no mesmo quadrado.

O Exemplo 1.1 com ações probabilísticas pode ser modelado por um MDP: em cada fase, o agente decide executar uma ação que o leva a um estado futuro, e ganha uma recompensa. O objetivo do agente é maximizar a recompensa esperada sobre uma sequência de ações. Como a solução para um MDP não é uma sequência fixa de ações, uma vez que há incerteza sobre os efeitos das ações, o objetivo do agente

\footnotetext{
${ }^{1}$ Inspirado no exemplo do Capítulo 17 em (Russell e Norvig 2010)
} 


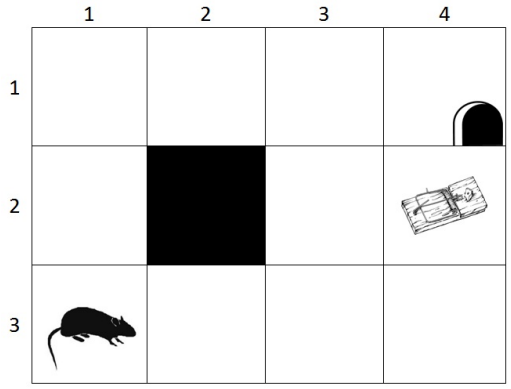

(a)

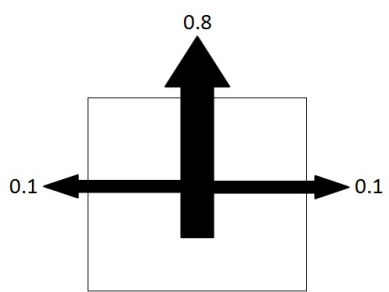

(b)

Figura 1.1: (a) Um problema $3 x 4$ do domínio “A fuga do rato”. (b) Ilustração da ação move_para_Norte: com probabilidade 0.8 o rato vai para o Norte e com 0.2 o rato se move em um ângulo reto em relação à direção pretendida.

é determinar a melhor ação para cada estado em que ele se encontra. Esta solução é chamada de política (Puterman 1994). A solução de um problema de planejamento é uma política ótima, ou seja, a política que associa a melhor ação para cada estado que o agente visitar. A maneira com que é determinada a política do agente é através da solução da equação de Bellman (Puterman 1994). Existem várias soluções para encontrar políticas ótimas, dentre elas se destacam as baseadas na programação dinâmica (Barto et al, 1995; Bonet e Geffner 2003; McMahan et al, 2005) e as que envolvem busca heurística no espaço de estados (Dai e Goldsmith 2007; Feng e Hansen, 2002; Hansen e Zilberstein 1999).

Um MDP requer uma representação precisa das probabilidades de transição e na maior parte dos problemas do mundo real, isso é impossível. Isto pode ocorrer por vários motivos: (i) informação imprecisa ou conflitante de especialistas nas medidas de probabilidades, (ii) existência de dados insuficientes usados para estimar o modelo preciso de transição de estados ou (iii) as probabilidades de transição são não-estacionárias devido à falta de informação sobre a dinâmica do processo (Delgado 2010).

Um Processo de Decisão Markoviano com Probabilidades Imprecisas (Markov Decision Processes with Imprecise Probabilities - MDP-IP), é um MDP em que as probabilidades são parametrizadas e sujeitas a um conjunto de restrições (Satia e Lave Jr, 1973). Isto permite que um MDP-IP represente crenças incompletas, ambíguas ou contraditórias do especialista sobre as probabilidades de transição.

Voltando ao Exemplo 1.1, suponha que a função de transição de estado seja alterada de forma que o efeito esperado por executar uma ação seja representado pelos parâmetros $p, q$ e $r$ e um conjunto de restrições sobre esses parâmetros. Essa situação está ilustrada na Figura 1.2. Essa nova função de transição de estado faz com que o problema se torne mais geral do que o anterior, dando uma maior flexibilidade na representação, possibilitando diferentes formulações para o problema.

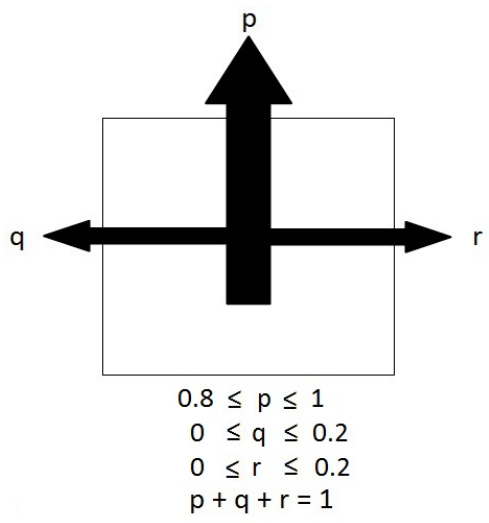

Figura 1.2: Função de transição de estado para a ação mover_para_Norte em que o efeito da ação é dado por probabilidades imprecisas representadas pelos parâmetros $p$, q e $r$, e o conjunto de restrições.

Uma solução para um MDP-IP é baseada na Teoria dos Jogos. O MDP-IP pode ser visto como um jogo entre duas entidades: o agente e a Natureza. 
É possível definir vários critérios de otimização para avaliar uma política (Delgado 2010), por exemplo, o critério maximin que assume que o agente tenta maximizar sua recompensa enquanto a Natureza tenta minimizá-la.

Jogos Markovianos (Markov Game - MG) estendem o modelo MDP para ambientes multiagentes (jogos). Uma solução para um jogo prescreve a melhor política para cada jogador. Frequentemente procura-se uma solução na qual as políticas estão em equilíbrio de Nash, isto é, nenhum jogador se beneficia em mudar sua própria política se todos os outros jogadores mantém suas próprias políticas de equilíbrio. Esse equilíbrio pode ser visto como um ótimo local no espaço de políticas.

Um tipo de jogo markoviano é o jogo markoviano alternado (Alternating Markov Game - AMG) (Littman 1996), no qual:

1. os jogadores alternam nas escolhas de suas ações (apenas um jogador realiza uma ação em cada estado), e

2. cada jogador conhece as jogadas realizadas pelos outros jogadores neste jogo, ou seja, o jogo é de informação perfeita (Parthasarathy e Raghavan 1971).

O Exemplo 1.2 ilustra um jogo markoviano alternado.

Exemplo 1.2 (Domínio do Gato e o Rato). Suponha que a ratoeira ilustrada no Exemplo 1.1 seja substituída por um gato. Agora o agente, representado pelo rato, tem de tomar as decisões para alcançar a meta, ou seja, o buraco, levando em consideração que há outro agente no ambiente. Nesse jogo o ambiente é completamente observável, pois o rato sabe em que posição o gato está. As ações para cada um dos jogadores são: mover_para_Norte, mover_para_Sul, mover_para_Oeste, mover_para_Leste e Noop. Os estados do jogo são caracterizados pelas posições de ambos os jogadores no ambiente e por um atributo que identifica de quem é a vez de escolher uma ação. O jogo é alternado e, portanto, os jogadores alternam na escolha de suas ações. Isso corresponde a um jogador executar uma ação em um dado estado e o outro jogador aplicar uma ação Noop. As ações tem efeitos probabilísticos.

$O$ gato recebe uma recompensa $R$ ao pegar o rato e o rato recebe $-R$. Mas se o rato conseguir chegar ao buraco sem ser pego, o gato recebe - $R$ e o rato recebe $R$. Dizemos que esse é um jogo de soma zero.

o jogo termina quando o gato pega o rato ou quando o rato consegue chegar ao buraco. Lembrando que o estado $(1,4)$ é um estado não alcançável pelo gato a partir do estado inicial, sendo assim, o gato não pode estar na posição em que há o buraco.

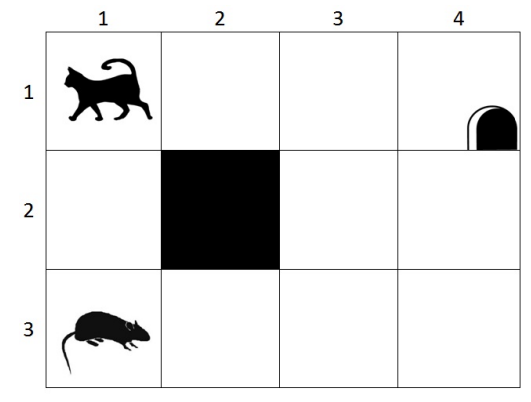

Figura 1.3: Exemplo de um possível estado do jogo do Gato e Rato definido no Exemplo 1.2, em que um dos jogadores é representado pelo gato, o outro jogador pelo rato e o buraco representa o estado meta do rato.

Se para o jogo do Exemplo 1.2 adotarmos a função de transição de estados da Figura 1.2, teremos um AMG com probabilidades imprecisas. Este novo AMG, chamado de AMG-IP, pode ser visto como uma extensão de um MDP-IP para ambientes multiagentes.

\subsection{Motivação}

Um modelo que permite representar as transições de estados com intervalos de probabilidades em jogos markovianos alternados foi proposto por Chang (2006). Porém, além de assumir apenas a imprecisão nas probabilidades dadas por intervalos, esse trabalho não explora diferentes critérios de preferência dos jogadores. 
Falta na literatura um trabalho que investigue diferentes soluções dependendo das suposições feitas sobre o comportamento da Natureza em jogos com probabilidades imprecisas. Assim, a motivação deste trabalho é estender o modelo proposto por Chang (2006) para imprecisões nas probabilidades dadas por parâmetros e um conjunto de restrições sobre eles, bem como investigar os diferentes critérios de preferência dos jogadores diante das escolhas da Natureza.

\subsection{Objetivos}

O objetivo principal deste trabalho é generalizar soluções para AMGs com probabilidades imprecisas. A primeira generalização é feita com base em trabalhos anteriores sobre MDP-IPs: um MDP em que as probabilidades de transição podem ser imprecisas, ou seja, ao invés de uma medida de probabilidade sobre o espaço de estados, temos um conjunto de medidas de probabilidade (Delgado et al, 2011). Assim, um dos objetivos desse trabalho é definir um modelo análogo para jogos markovianos alternados, que chamaremos de jogos markovianos alternados com probabilidades imprecisas dadas por conjuntos credais (Alternating Markov Games with Imprecise Probabilities - AMG-IP) e discutir diferentes critérios de preferência dos jogadores diante das escolhas da Natureza. A segunda generalização dos AMGs é feita sobre o MDPST (Markov Decision Process with Set-valued Transitions), uma subclasse de problemas MDP-IP em que as transições probabilísticas são feitas sobre um conjunto de estados. Chamamos esse novo modelo de AMG-ST (Alternating Markov Game with Set-valued Transitions), sendo que as soluções propostas para os AMG-IPs também valem para os AMG-IPs.

\subsection{Principais contribuições}

Com o objetivo de estudar as relações entre MDPs e AMGs, esse trabalho tem como principais contribuições:

- Formalização de um novo modelo AMG com probabilidades imprecisas - AMG-IP. Com base no trabalho de Chang (2006), que propôs um AMG com probabilidades imprecisas dadas por intervalos, propomos um novo modelo chamado de Jogo Markoviano Alternado com Probabilidades Imprecisas (AMG-IP) que é uma extensão de MDP-IPs para jogos em que a função de transição de estados tem seus valores de probabilidades parametrizadas e é dado um conjunto de restrições sobre eles, sendo que o conjunto de valores reais que satisfazem as restrições define um conjunto credal;

- Formalização de dois novos modelos de jogos com transições valoradas por conjunto - AMG-ST e AMG-ST-1. Foram propostos ainda dois novos modelos de AMGs com imprecisão: o AMG-ST, uma classe especial de AMG-IP em que as transições vão probabilisticamente para conjuntos de estados; e o AMG-ST-1 em que somente um jogador faz transição para conjuntos de estados e o segundo jogador seleciona um estado desse conjunto. Enquanto no AMG-IP e AMG-ST a Natureza não é modelada como um agente, no AMG-ST-1 ela faz o papel do Jogador II.

- Estudo sobre diferentes critérios de preferência dos jogadores diante das escolhas da Natureza para solução de AMG-IPs. A solução de um AMG-IP é baseada nas relações de ordem entre intervalos da função valor de estado, isso porque diante das escolhas da Natureza os jogadores podem fazer suposições pessimistas e otimistas sobre essas escolhas. Entre as soluções propostas neste trabalho temos:

- Upper-First: "dentre os pares de políticas ótimas de equilíbrio supondo a Natureza cooperativa, escolhemos um par de políticas de equilíbrio assumindo agora a Natureza adversarial". Esse foi o critério proposto por Chang (2006), porém, adotamos uma definição de equilíbrio diferente e o comparamos a um novo critério similar: o Lower-First.

- Lower-First: "dentre os pares de políticas ótimas de equilíbrio supondo a Natureza adversarial, escolhemos um par de políticas de equilíbrio assumindo agora a Natureza cooperativa". Esse é um critério robusto e provamos que os pares de políticas encontrados por esse critério estão em equilíbrio. Com essa nova solução mostramos uma variação importante do trabalho de Chang (2006). Comparamos essa solução com o critério Upper-First e verificamos que esses dois critérios podem devolver pares de políticas diferentes. 
- $\epsilon$-Lower-First e $\epsilon$-Upper-First: esses critérios permitem uma tolerância $\epsilon$ na primeira fase das soluções Lower-First e Upper-First e assim, é possível obter uma recompensa média esperada maior que a solução de Chang (2006) (Upper-First), e o critério Lower-First. Assim, mostramos que os critérios $\epsilon$-Lower-First e $\epsilon$-Upper-First encontram políticas melhores ou iguais que os pares de políticas encontrados pelos critérios Lower-First e Upper-First, respectivamente.

- $\eta$-optimal: esse critério de preferência permite uma maior flexibilidade sobre as suposições de escolhas da Natureza, considerando uma combinação linear entre os limites inferiores e superiores dos intervalos da função valor. Dependendo do valor assumido por um parâmetro $\eta$, chamado de parâmetro de precaução, que pode variar entre 0 e 1 , o jogador pode definir uma medida de equilíbrio entre uma suposição mais otimista ou pessimista sobre o comportamento da Natureza.

\subsection{Trabalhos correlatos}

Jogos markovianos alternados de dois jogadores de soma zero são amplamente estudados na literatura de teoria dos jogos, sendo que os resultados fundamentais da área foram obtidos a partir de trabalhos em MDPs (Puterman 1994). Kurano et al. (1998) generalizaram o modelo MDP em que a probabilidade de transição varia a cada estágio e demonstraram que seu modelo converge para uma solução ótima. O modelo proposto por Kurano et al. (1998) está relacionado a um MDP-IP (Delgado et al, 2011; Satia e Lave Jr, 1973; White III e Eldeib 1994) e a um BMDP (Bounded-parameter Markov Decision Processes) (Givan et al. , 2000). Chang (2006) estendeu o trabalho de Kurano para resolver AMGs de dois jogadores de soma zero com imprecisão nas probabilidades dadas por intervalos e provou que é possível encontrar políticas de equilíbrio para esse jogo. A nossa extensão foi inspirada no modelo MDP-IP (Delgado et al, 2011). Sendo este um modelo mais geral que os modelos MDP e BMDP, ao estendermos o MDP-IP para dois jogadores, definimos um tipo mais geral de AMG, que chamamos de AMG-IP. O trabalho de Quaeghebeur e de Cooman (2009) também inclui imprecisão em um jogo de dois jogadores e o principal objetivo é fazer com que o Jogador I aprenda a estratégia do Jogador II (adversário) usando o modelo de Dirichlet impreciso para representar e atualizar as crenças do Jogador I, porém o trabalho trata de jogos simultâneos com políticas probabilísticas, enquanto que estamos interessados em jogos alternados com políticas determinísticas.

A teoria de jogos com espaço de estados finito foi muito explorada por Shapley (1953) e seus resultados foram estendidos para jogos com espaço de estados infinitos (Bewley e Kohlberg 1978). Visto que os problemas de planejamento em jogos, assim como em MDPs, também sofrem com o problema da explosão de estados, algumas abordagens e algoritmos vem sendo propostos para contornar este problema. Kearns et al. (2000), por exemplo, fornecem uma generalização do algoritmo de iteração por valor de horizonte finito para jogos markovianos e utiliza-o para construir um algoritmo que computa uma estratégia aproximada para grandes ou infinitos espaços de estados. Já o trabalho de Lagoudakis e Parr (2002) propõe um algoritmo baseado em iteração por política para aproximar a função valor em jogos markovianos. Além das técnicas de aproximação da função valor, também há soluções baseadas na técnica de agregação de estados (Huang et al, 2004), como o trabalho de Chang (2006) que combina o modelo AMG (Alternating Markov Game) com o modelo BMDP (Givan et al, 1997) e apresenta um modelo de jogo markoviano alternado de dois jogadores de soma zero com probabilidades de transição imprecisas, além de estabelecer uma política de equilíbrio para esse modelo. De acordo com Chang (2006), o modelo pode ser aplicado em jogos markovianos alternados em que as probabilidades de transição são perturbadas ou em jogos markovianos em que as soluções são aproximadas por agregações de estados, visto que, dessa forma, grandes espaços de estados podem ser reduzidos a espaços de estados menores.

A literatura de jogos markovianos é bem extensa e varia entre jogos alternados e simultâneos. Apesar do modelo de jogo simultâneo ser diferente do modelo de jogo alternado, Altman et al. (1996) mostrou que um jogo alternado de dois jogadores pode ser transformado em um jogo simultâneo em que um dos jogadores sabe exatamente quais serão as ações escolhidas pelo outro jogador. Isso permite que o conjunto de estados dos agentes não seja disjunto e que, num mesmo estado, ambos os jogadores escolham suas ações, assim como na solução proposta em Littman (1994) para resolver jogos markovianos alternados a partir da solução de jogos markovianos simultâneos. 


\subsection{Organização}

Este trabalho está organizado da seguinte forma:

- No Capítulo 2 discutimos os fundamentos do trabalho. Assim, formalizamos o Processo de Decisão Markoviano, visto que é o modelo base para todos os outros modelos estudados neste trabalho. Neste capítulo também apresentamos o Processo de Decisão Markoviano com Probabilidades Imprecisas, assim como suas variantes, o BMDP e o MDP-ST. Esses modelos são extensões do MDP que permitem que seja inserida imprecisão nas probabilidades de transição de estados e estão diretamente relacionados aos modelos estudados nos Capítulos seguintes. E, finalmente, descrevemos os jogos markovianos alternados e mostramos como calcular a solução para tais jogos.

- No Capítulo 3 mostramos a correspondência entre um MDP-ST e um AMG em que o jogador adversário é a Natureza e apresentamos uma extensão do modelo MDP-ST estudado no Capítulo 2 para jogos markovianos alternados.

- No Capítulo 4 apresentamos os AMGs com imprecisão nas probabilidades (AMG-IP), mostramos a prova de equilíbrio para este modelo e os vários critérios de preferência dos jogadores diante das escolhas da Natureza para solucionar os AMG-IPs, além de descrevermos algoritmos para resolvê-los usando cada critério discutido.

- No Capítulo 5 apresentarmos os experimentos realizados a partir de um exemplo como prova de conceito dos critérios propostos.

- No Capítulo 6 discutimos as conclusões e os possíveis trabalhos futuros. 


\section{Capítulo 2}

\section{Fundamentos}

\subsection{Processos de Decisão Markovianos}

Um Processo de Decisão Markoviano (Markov Decision Process - MDP) modela sistemas dinâmicos em que as transições entre estados são probabilísticas. Cada ação tem uma recompensa e/ou custo, que depende do estado em que o processo se encontra. Como o efeito de uma ação em um estado depende apenas da ação e do estado atual do sistema, tais processos são ditos Markovianos (Howard 1960.).

Formalmente, um processo de decisão markoviano é definido como a seguir.

Definição 2.1 (MDP). Um MDP é uma tupla $M=(S, A, R, p, \gamma)$, em que (Delgado 2010; Howard 1960.; Littman 1996):

- S é um conjunto discreto e finito de estados completamente observáveis que modelam o ambiente.

- A é um conjunto finito de ações; a execução de uma ação permite que o sistema mude do estado atual para o próximo estado.

- $R: S \times A \rightarrow \mathbb{R}$ é uma função recompensa associada ao agente por estar em um determinado estado e executar uma ação.

- $p: S \times A \times S \rightarrow P D(S)$ define a probabilidade de transição, em que $P D(S)$ representa o conjunto de distribuição de probabilidades sobre $S$. Assim, $p\left(s^{\prime} \mid s, a\right)$ é a probabilidade condicional de ir para $o$ estado $s^{\prime} \in S$ quando o agente está no estado $s \in S$ e executa a ação $a \in A$. Note que a função de transição satisfaz a suposição de Markov, ou seja, a probabilidade de ir para o estado s' a partir de $s$ depende unicamente de s, i.e., independe dos estados anteriores.

- $\gamma$ é um fator de desconto, sendo $0 \leq \gamma<1$ para cada ação executada e depois de t estágios a recompensa é descontada por $\gamma^{t}$.

Assim, o problema de planejamento probabilístico é escolher a melhor ação para ser executada a cada estado.

\subsubsection{Função valor e política ótima}

Como as transições de estados são probabilísticas, não é possível determinar uma sequência fixa de ações a ser seguida como no planejamento clássico. Assim, as ações a serem executadas pelo agente são determinadas por funções conhecidas como políticas, $\pi: S \rightarrow A$, que associam uma ação para cada estado visitado pelo agente.

Um agente pode seguir uma política por um tempo (número de passos) finito ou infinito. Caso o agente siga a política por um número finito de passos, a maneira como o agente se comporta costuma mudar na medida em que ele se aproxima de seus últimos passos. Neste caso, a política é não-estacionária. Caso contrário, ou seja, quando o agente segue a política por um número infinito de passos, não há porque o agente mudar seu modo de agir com o passar do tempo. Assim, é mais razoável considerar $\pi$ uma política estacionária e costuma-se utilizar uma taxa de desconto $\gamma$ com valores entre 0 e 1 para garantir que o valor alcançado por essa política seja finito (Pereira e de Barros 2007). Dessa forma, a política $\pi$ é chamada de estacionária se ela não altera em função do tempo. 
Neste trabalho, assumimos MDPs de horizonte infinito e que as políticas são estacionárias. Dessa forma, uma política estacionária $\pi: S \rightarrow A$ especifica a ação $a=\pi(s)$ a ser escolhida em cada estado $s$.

O objetivo do agente MDP é encontrar a política que maximiza a soma esperada da recompensa descontada num horizonte infinito. O valor da política $\pi$ começando no estado $s$ e executando $\pi(s)$, denotado por $V_{\pi}(s)$ - chamada de função valor -, é definido como a soma esperada das recompensas descontadas sob um horizonte infinito, ou seja (Puterman 1994):

$$
V_{\pi}(s)=R(s, \pi(s))+\gamma \sum_{s^{\prime} \in S} p\left(s^{\prime} \mid s, \pi(s)\right) V_{\pi}\left(s^{\prime}\right) .
$$

Por outro lado, a função valor ótima, representada por $V^{\star}(s)$, é a função valor associada com qualquer política ótima. Assim, para um agente que deseja maximizar sua recompensa esperada, $V^{*}(s)$ satisfaz a seguinte igualdade, conhecida como equação de Bellman (Puterman 1994):

$$
V^{*}(s)=\max _{a \in A}\left(R(s, a)+\gamma \sum_{s^{\prime} \in S} p\left(s^{\prime} \mid s, a\right) V^{*}\left(s^{\prime}\right)\right) .
$$

A Equação (2.2) é a solução para MDPs e sua prova está baseada no conceito de contração e convergência (Puterman 1994).

\subsubsection{Prova de convergência}

Uma forma de mostrar que a Equação (2.2) é a solução de um MDP é usando o teorema de contração de ponto fixo de Banach (Puterman 1994).

Teorema 2.1 (Teorema do ponto fixo de Banach). Se $f: X \rightarrow X$ é uma contração (contraction mapping) de um espaço métrico completo $X$ para ele mesmo, então $f$ tem um único ponto fixo.

Se $X$ é um espaço de Banach, então um operador $f: X \rightarrow X$ é uma contração (contraction mapping) se existe um $\lambda$ tal que $0 \leq \lambda<1$ e $\|f v-f u\| \leq \lambda\|v-u\|$ para todo $u$ e $v$ em $X$ (Givan et al, 2000; Puterman, 1987).

Sendo assim, seja $F(S)$ o espaço de funções reais em $S$ dotado com a norma máxima

$$
\|v\|=\max _{s \in S}|v(s)| .
$$

O espaço $F(S)$ junto com \|. $\|$ constitui um espaço linear completo e normado ou Espaço de Banach.

Usamos o teorema do ponto fixo de Banach (Teorema 2.1) para mostrar que iterativamente estes operadores convergem para pontos fixos únicos, $V_{\pi}$ e $V^{*}$. Para isso, definimos os operadores $T: F(S) \rightarrow F(S)$ e para cada $\pi \in \Pi, T_{\pi}: F(S) \rightarrow F(S)$ em cada $s \in S$ por

$$
T(v)(s)=\max _{a \in A}\left(R(s, a)+\gamma \sum_{s^{\prime} \in S} p\left(s^{\prime} \mid s, a\right) v\left(s^{\prime}\right)\right)
$$

$\mathrm{e}$

$$
T_{\pi}(v)(s)=R(s, \pi(s))+\gamma \sum_{s^{\prime} \in S} p\left(s^{\prime} \mid s, \pi(s)\right) v\left(s^{\prime}\right) .
$$

Usando estes operadores, podemos reescrever as definições de $V^{*}$ e $V_{\pi}$ como

$$
V^{*}(s)=T(v)(s) \quad \text { e } \quad V_{\pi}(s)=T_{\pi}(v)(s)
$$


Os quatro teoremas a seguir, retirados de Puterman (1987) e Givan et al. (2000), mostram que cada operador de contração aplicado à função valor, convergem iterativamente para pontos fixos.

Teorema 2.2. Para qualquer espaço de Banach $X$ e mapeamento de contração $f: X \rightarrow X$, existe um único $v^{*}$ em $X$ tal que $f v^{*}=v^{*}$ e para um $v^{0} \in X$ arbitrário, a sequência $\left\{v^{n}\right\}$ definida por $v^{n}=f v^{n-1}=f^{n} v^{0}$ converge para $v^{*}$.

Teorema 2.3. $T(v)$ e $T_{\pi}(v)$ são mapeamentos de contração.

Teorema 2.4. Existe um único $v^{*} \in F(S)$ satisfazendo $v^{*}=T\left(v^{*}\right)$, além disso, $v^{*}=V^{*}$. Similarmente, $V_{\pi}$ é um ponto fixo de $T_{\pi}$.

Teorema 2.5. Para um $v^{0} \in F(S)$, a sequência $\left\{v^{n}\right\}$ definida por $v^{n}=T\left(v^{n-1}\right)=T^{n}\left(v^{0}\right)$ converge para $V^{*}$. Similarmente, iterando $T_{\pi}$ converge para $V_{\pi}$.

Uma consequência importante do Teorema 2.5 é que ele fornece um algoritmo para encontrar $V^{*}(s)$ e $V_{\pi}(s)$. Em particular, para encontrar $V^{*}(s)$ podemos começar a partir de uma função valor $v^{0}$ em $F(S)$ arbitrária e aplicar repetidamente o operador $T$ para obter a sequencia $\left\{v^{n}\right\}$. Este algoritmo é conhecido como Iteração de Valor (Value Iteration). Similarmente, podemos especificar um algoritmo chamado de Iteração de Política (Policy Iteration) que encontra $V_{\pi}$ aplicando repetidamente o operador $T_{\pi}$, começando com um valor inicial $v^{0} \in F(S)$.

\subsubsection{Métodos computacionais para resolver um MDP}

O algoritmo de Iteração de Valor é um método de aproximação que usa programação dinâmica, enquanto que o algoritmo de Iteração de Política é exato. Para estudar a convergência de um método de aproximação, devemos ter uma noção de distância. Se $v$ é uma função real valorada em $S$, a norma de $v$, denotada por $\|v\|$ é definida na Equação (2.3). A distância entre dois vetores $v$ e $u$ é dada por $\|v-u\|$. Isto significa que dois vetores estão $\epsilon$ unidades separados se a diferença máxima entre qualquer dois componentes é $\epsilon$ unidades. Assim, uma política $\pi$ é dita ser $\epsilon$-ótima se $\left\|v^{\pi}-v^{*}\right\|<\epsilon$. Se $\epsilon$ é suficientemente pequeno, os dois algoritmos podem ser usados para encontrar políticas cuja recompensa total descontada é arbitrariamente próxima do ótimo (Puterman 1987).

\section{Iteração de Valor}

O Algoritmo 1 (MDP-VALUE-ITERATION) é um algoritmo de programação dinâmica que implementa o algoritmo de Iteração de Valor. Dado um vetor $v$ de tamanho $|S|$, representando a função valor para todos os estados $s \in S$ de um MDP e $\epsilon>0$, o algoritmo de Iteração de Valor obtém uma política $\epsilon$-ótima (Puterman 1987).

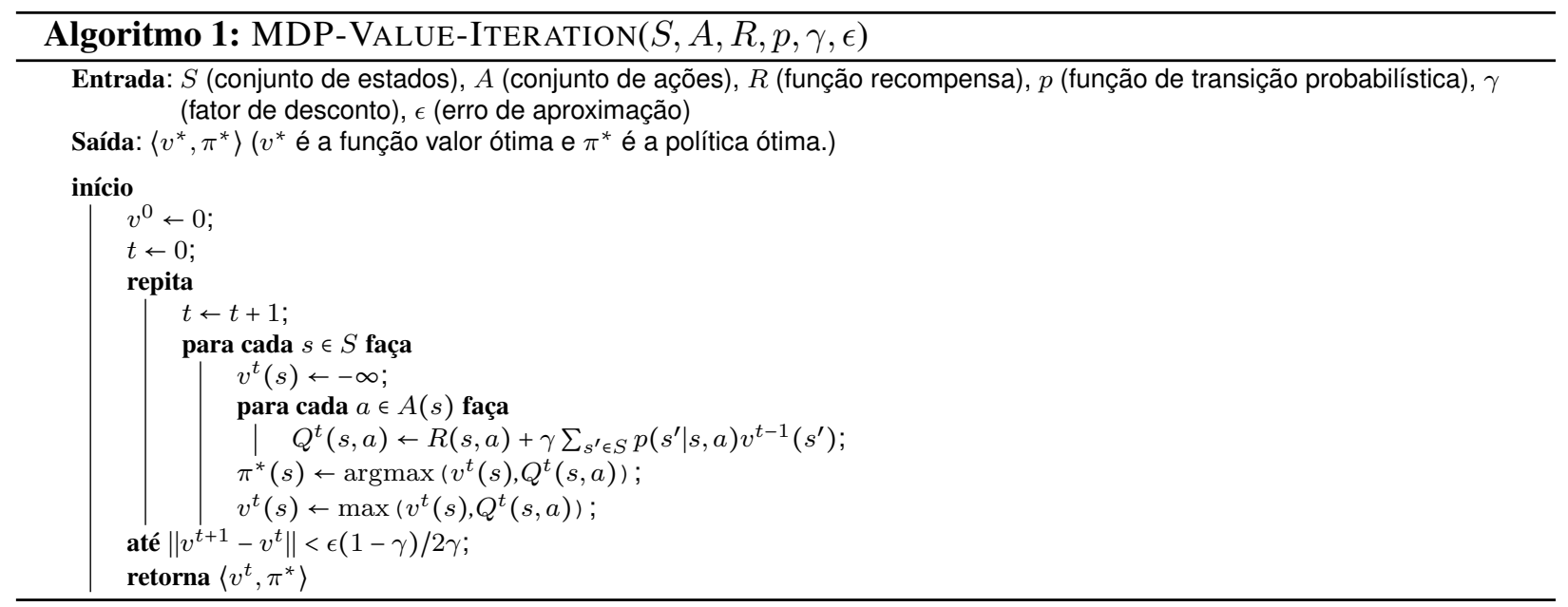




\section{Iteração de Política}

O Algoritmo 2 (MDP-PoliCY-ITERATION) (Pereira e de Barros 2007) implementa o algoritmo de Iteração de Política e usa uma estrutura especial de problemas estacionários de programação dinâmica de horizonte infinito para encontrar todas as políticas ótimas. O algoritmo é dividido em duas partes principais, um passo chamado de avaliação de política e um passo chamado de melhoria de política (Puterman 1994). Ele termina quando o conjunto de ações maximizadoras encontrado no estágio de melhora se repete, isto é, se a mesma política é obtida em dois passos sucessivos através do laço de iteração principal.

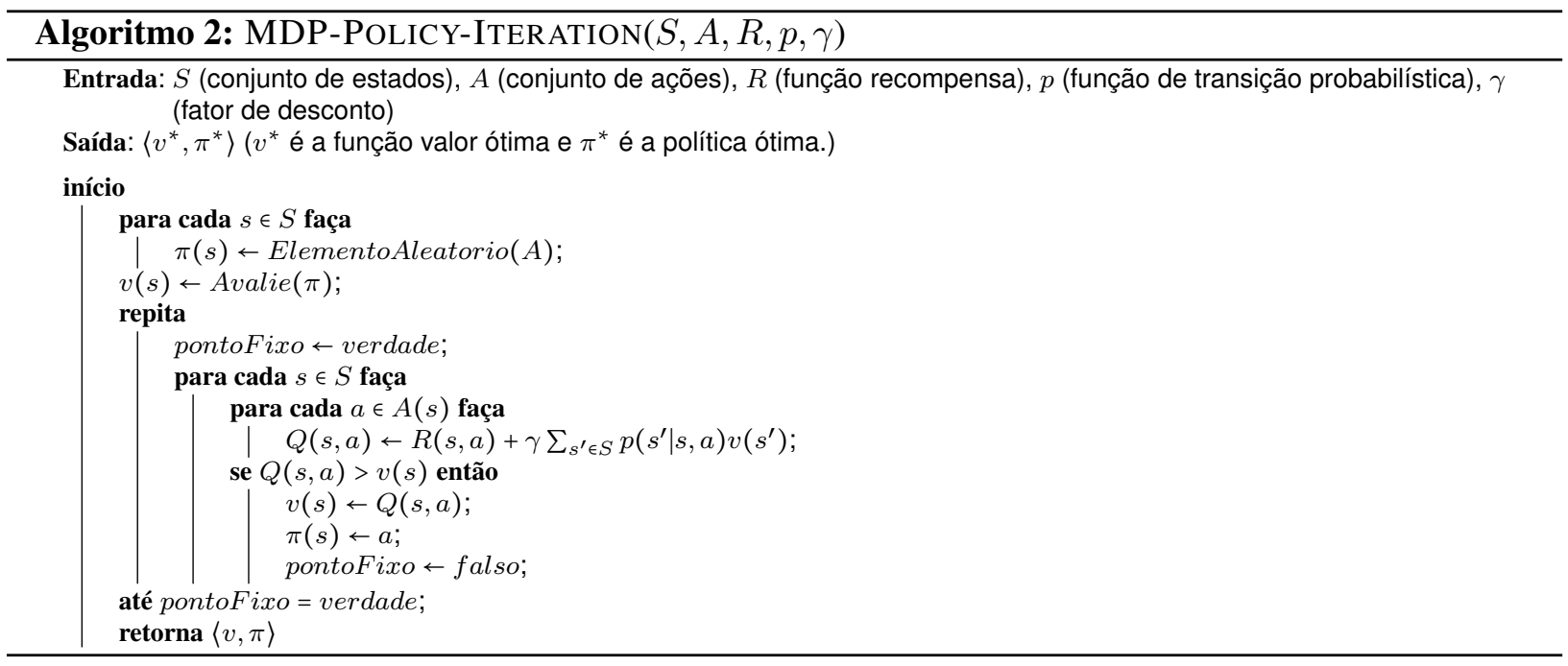

Programação Dinâmica em Tempo Real Para domínios em que a função de transição de estados é não densa e o estado inicial é conhecido, algoritmos baseados em técnicas de amostragem podem ser mais eficientes que os algoritmos de Iteração de Valor ou Iteração de Política. O algoritmo RTDP (Real Time Dynamic Programming), proposto por Barto et al. (1995), calcula a função valor $V^{*}\left(s_{0}\right)$ considerando apenas os estados visitados a partir de $s_{0}$. Versões estendidas da RTDP estão entre os algoritmos considerados o estado da arte das soluções para MDPs (Bonet e Geffner 2003; Kolobov et al, 2012; McMahan et al, 2005; Trevizan e Velose 2012).

\subsection{Processo de Decisão Markoviano com Probabilidades Imprecisas}

Um MDP requer uma representação precisa das probabilidades de transição e na maior parte dos problemas do mundo real, isso é praticamente impossível. Isto pode ocorrer por vários motivos: (i) informação imprecisa ou conflitante de especialistas nas medidas de probabilidades, (ii) existência de dados insuficientes usados para estimar o modelo preciso de transição de estados ou (iii) as probabilidades de transição são não-estacionárias devido à falta de informação sobre a dinâmica do processo (Delgado 2010; Givan et al. , 2000; Trevizan et al, 2007).

Algumas extensões foram propostas com o objetivo de permitir tipos diferentes de incerteza sobre a função de transição de estados, entre elas:

- o processo de decisão markoviano com probabilidades imprecisas dadas por intervalos (Boundedparameter Markov Decision Process - BMDP);

- o processo de decisão markoviano com transição valorada por conjunto (Markov Decision Process with Set-Valued Transition - MDPST);

- o processo de decisão markoviano com probabilidades imprecisas dadas por conjuntos credais (Markov Decision Process with Imprecise Probabilities - MDPIP), que generaliza os dois modelos anteriores. 


\subsubsection{Processo de Decisão Markoviano com Probabilidades Imprecisas dadas por Intervalos - BMDP}

O BMDP (Bounded-parameter Markov Decision Process) pode ser visto como uma generalização dos MDPs, de forma que um BMDP é um conjunto de MDPs (todos os MDPs deste conjunto compartilham o mesmo espaço de estados e de ações e só diferem na função de transição probabilística). A principal diferença entre um BMDP e um MDP é que, ao invés de haver um número real entre 0 e 1 representando uma probabilidade de transição de estado, há um intervalo fechado de números reais. Por exemplo, dado um conjunto de estados $S$, a probabilidade de ir para o estado $s^{\prime} \in S$ estando em $s \in S$ e executando a ação $a$ é dada pelo intervalo $\left[p\left(s^{\prime} \mid s, a\right), \bar{p}\left(s^{\prime} \mid s, a\right)\right] \subseteq[0,1]$. Chamamos de $p(. \mid s, a)$ a distribuição de probabilidade de ir para os estados de $S$ dado que o agente está em $s$ e executa a ação $a$ (o "." representa todos os estados de $S$ ). Uma vez que as probabilidades de transição de estados são dadas na forma de intervalos, a função de transição de estados também é definida por intervalos.

Definiçãa 2.2 (Função de transição intervalar). $\hat{P}=\langle\underline{p}, \bar{p}\rangle$ é uma função de transição intervalar em que $p=p(. \mid s, a) \in \mathbb{R}^{1 \times|S|}$ e $\bar{p}=\bar{p}(. \mid s, a) \in \mathbb{R}^{1 \times|S|}$ representam os conjuntos de vetores de tamanho $|S|$ que

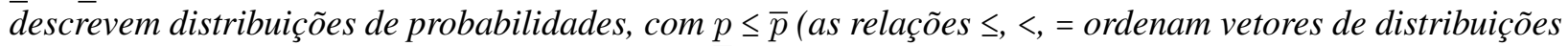
de probabilidades e são definidas ao longo do texto para as diferentes soluções apresentadas nessa dissertação) $e\langle p, \bar{p}\rangle \neq \varnothing$, em que $\langle p, \bar{p}\rangle=\{p \mid p$ é uma distribuição de probabilidade sobre $S$ com $p \leq p \leq \bar{p}\}$. Definimos $d(\bar{s}, a)=\langle p(. \mid s, a), \bar{p}(. \mid s, a)\rangle$ como o conjunto de distribuições de probabilidade associado ao estado s e ação a (Chäng 2006; Kurano et al, 1998).

Definição 2.3 (BMDP (Givan et al, 2000)). Um Processo de Decisão Markoviano com Probabilidades Imprecisas dadas por Intervalos é definido pela tupla $\langle S, A, \hat{P}, R, \gamma\rangle$ em que:

- S é o conjunto finito e discreto de estados, tal qual como em um MDP;

- A é o conjunto finito e discreto de ações;

- $\hat{P}=\langle\underline{p}, \bar{p}\rangle$ é uma função de transição intervalar (interval transition function);

- $R: S \times A \rightarrow \mathbb{R}$ é a função recompensa;

- $\gamma$ é um fator de desconto.

A Figura 2.1 exibe um diagrama de transição de estados para um BMDP com três estados e uma ação. Note que ao aplicar a ação $a$ no estado $s_{1}$ com probabilidade entre 0.7 e 0.8 o agente vai para o estado $s_{2}$ e com probabilidade entre 0.2 e 0.5 , o agente vai para o estado $s_{3}$. Note que os intervalos de uma dada transição de estado usando uma determinada ação são ditos serem válidos se a soma dos limites inferiores destes intervalos for menor ou igual a um e a soma dos limites superiores for maior ou igual a um.

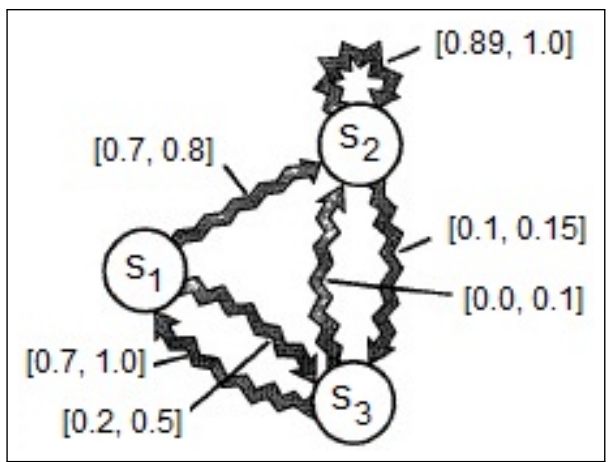

Figura 2.1: Diagrama de transição de estados para um BMDP simples com três estados e uma única ação sendo aplicada aos três estados. Os arcos indicam possíveis transições e são rotulados por seus limites inferiores e superiores de probabilidades. Fonte: Givan et al. (2000). 
Solução robusta para o BMDP Como a distribuição de probabilidades $p(. \mid s, \pi(s)) \in d(s, \pi(s))$ pode ser escolhida de forma arbitrária a partir dos conjuntos de distribuições de probabilidade $d(s, \pi(s))$, dizemos que essa é uma escolha da Natureza. Assim, uma solução robusta (ou pessimista) é assumir que a Natureza é adversária e irá selecionar uma distribuição de probabilidades que minimize a recompensa acumulada futura, enquanto o agente quer maximizá-la (critério maxmin).

Analogamente às soluções ótimas para MDPs (Equação (2.2)), podemos calcular o valor ótimo da função valor considerando a Natureza adversária usando a equação:

$$
\underline{V}^{*}(s)=\max _{a \in A}\left[R(s, a)+\gamma \min _{p \in d(s, a)} \sum_{s^{\prime} \in S} p\left(s^{\prime} \mid s, a\right) \underline{V}^{*}\left(s^{\prime}\right)\right] .
$$

Existem soluções eficientes para BMDPs baseadas no algoritmo RTDP (Real Time Dynamic Programming) (Barto et al, 1995), chamadas de Robust LRTDP (Buffet e Aberdeen 2005).

Uma aplicação interessante para um BMDP é interpretá-lo como um modelo resultante de um processo de agregação de estados de um MDP. Dessa forma, os estados do BMDP representam conjuntos (agregados) de estados que foram agrupados. Os intervalos, portanto, representam as variações dos parâmetros sobre os estados pertencentes à agregação. Assim, ao invés de interpretarmos os estados de um BMDP como estados individuais, podemos interpretá-los como um conjunto de estados, ou estados agregados, de um grande MDP (Givan et al, 1997).

\subsubsection{Processo de Decisão Markoviano com Transição Valorada por Conjunto - MDP-ST}

O processo markoviano de decisão com transição valorada por conjunto (Markovian Decision Process with Set-valued Transition - MDP-ST) foi proposto por Trevizan (2006) para modelar problemas de planejamento com um tipo mais geral de incerteza do que a encontrada no modelo MDP e diferente daquela modelada por um BMDP.

Um MDP-ST combina duas formas de incerteza. Nos modelos não-determinísticos, a execução de uma ação pode levar o sistema a diferentes estados, sem que haja alguma preferência entre eles (simplesmente por desconhecê-los, por exemplo). Por outro lado, os modelos probabilísticos são caracterizados quando são conhecidas as probabilidades sobre os efeitos das ações. Dizemos que a execução de ações no primeiro modelo está associada à "Incerteza Knightiana" e no segundo modelo, ao Risco (Trevizan 2006).

Os MDP-STs fornecem uma semântica precisa para problemas de planejamento com ações não determinísticas e probabilísticas, bem como para a combinação de ambas em qualquer proporção. Para isso é definida uma nova função de transição $p$, baseada na ideia de transição para conjunto de estados alcançáveis em um passo.

Um conjunto de estados alcançáveis em um passo é um conjunto de estados, unitário ou não, para o qual está associado um valor de probabilidade. Denotamos os conjuntos alcançáveis por $k \subseteq S$ em que $S$ é o conjunto de estados do MDP-ST. Dessa forma, a função de probabilidade condicional $p(k \mid s, a)$ em que $k \subseteq S$ é definida por $p: S \times A \times 2^{S} \backslash \varnothing \rightarrow P D\left(2^{S}\right)$. Note que ainda há incerteza sobre o valor de $p(k \mid s, a)$ para cada estado sucessor $s^{\prime} \in k$. Assim, a semântica dessa nova função de transição probabilística é: o conjunto de estados alcançáveis $k \subseteq S$ será o resultado de aplicar a ação $a$ no estado $s$ com probabilidade $p(k \mid s, a)$ e o estado resultante $s^{\prime} \in k$ será escolhido de forma não-determinística entre os estados de $k$ (Trevizan 2006). A função $p(k \mid s, a)$ é comumente denotada por função de atribuição de massa de probabilidade. A Figura 2.2 ilustra um exemplo de MDP-ST. Note que quando o agente está num estado $s$ e escolhe uma ação $a_{1}$, com probabilidade 0.3 , ele faz uma transição para o estado $s_{1}$ ou $s_{2}$ e com probabilidade 0.7 para o estado $s_{3}$ ou $s_{4}$. Dizemos que as escolhas entre $s_{1}$ ou $s_{2}$ e $s_{3}$ ou $s_{4}$ são escolhas da Natureza.

Definição 2.4 (MDP-ST). Um MDP com transições valoradas por conjuntos é definido pela tupla $\langle S, A$, $F, p, R, \gamma\rangle$, em que (Trevizan 2006):

- S é um conjunto finito de estados;

- A é o conjunto de ações e $A(s) \subseteq A$ representa as ações aplicáveis no estado s; 


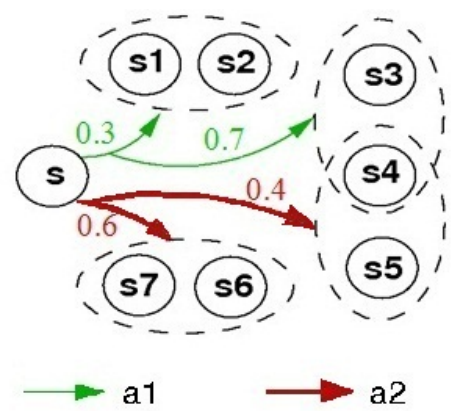

Figura 2.2: Exemplo de um MDP-ST. A ação $a_{1}$ vai com probabilidade 0.3 para o conjunto $\left\{s_{1}, s_{2}\right\}$ e com probabilidade 0.7 para o conjunto $\left\{s_{3}, s_{4}\right\}$.

- $F: S \times A \rightarrow 2^{S} \backslash\{\varnothing\}$, ou seja, $F(s, a) \subset 2^{S}$ que mapeia o estado s e a ação a para um conjunto $k$ não vazio de estados. Chamamos o conjunto $k$ de conjunto de estados alcançáveis em um passo pela ação a em s;

- $p: S \times A \times 2^{S} \rightarrow P D\left(2^{S}\right)$ é a função de atribuição de massa sobre $2^{S} \backslash\{\varnothing\}$ em que $p(k \mid s, a)$ representa o valor (massa) de probabilidade que deve ser repartido de qualquer maneira entre os elementos de $k$, após aplicar a ação a no estado s. $P D\left(2^{S}\right)$ representa o conjunto de distribuições de probabilidades sobre $2^{S}$;

- $R: S \times A \rightarrow \mathbb{R}$ é a função recompensa, em que $R(s, a)$ representa a função recompensa associada ao agente por estar no estado se executar a ação a.

- $\gamma$ é o fator de desconto, tal qual definido em MDP, sendo $0 \leq \gamma<1$; assim, depois de testágios a recompensa é descontada por $\gamma^{t}$.

Solução robusta para MDP-ST Assumindo que a escolha de estados em $k$ (escolha não determinística) é feita pela Natureza, (Trevizan et al, 2007) propôs uma solução robusta (ou pessimista) que considera a Natureza como um adversário que tenta minimizar os valores dos estados futuros. Assim, o valor da política $\pi$ começando no estado $s$ e executando $\pi(s)$ é dado por (Trevizan 2006):

$$
V_{\pi}(s)=R(s, a)+\gamma \min _{s^{\prime} \in k} \sum_{k \in F(s, \pi(s))} p(k \mid s, \pi(s)) V_{\pi}\left(s^{\prime}\right) .
$$

De maneira análoga à solução de um MDP (Equação (2.2)) temos que a função valor ótima, representada por $V^{\star}(s)$, é dada por:

$$
V^{*}(s)=\max _{a \in A(s)}\left(R(s, a)+\gamma \min _{s^{\prime} \in k} \sum_{k \in F(s, a)} p(k \mid s, a) V^{*}\left(s^{\prime}\right)\right)
$$

Trevizan et al. (2007) mostraram que essa solução pode ser modificada para:

$$
V^{*}(s)=\max _{a \in A(s)}\left(R(s, a)+\gamma \sum_{k \in F(s, a)} p(k \mid s, a) \min _{s^{\prime} \in k} V^{*}\left(s^{\prime}\right)\right)
$$

sendo mais eficiente que a anterior uma vez que a minimização é local (isto é, dentro da somatória).

Assim como num BMDP, um MDP-ST pode ser visto como um caso especial de jogo estocástico alternado cujo primeiro jogador é o sistema de tomada de decisão e o segundo jogador, a Natureza adversária. No Capítulo 3 discutimos essa equivalência e como a partir dela é possível provar a Equação 2.9.

\subsubsection{Processo de Decisão Markoviano com Probabilidades Imprecisas - MDP-IP}

Uma outra forma mais geral de definir MDPs com Probabilidades Imprecisas é impor um conjunto de restrições sobre os valores das probabilidades de transição a partir do estado $s$ para estado $s^{\prime}$. Tais restrições podem ser definidas como intervalos (Figuras 2.3a) como num BMDP, mas também podem ser mais gerais 
como na Figura 2.3b, que ilustra um exemplo de transições probabilísticas parametrizadas por $p, q$ e $r$. Uma distribuição de probabilidades é escolhida de tal forma que satisfaça o conjunto de restrições sobre os parâmetros $p, q$ e $r$. O conjunto de todas as distribuições de probabilidade que satisfazem um conjunto de restrições é chamado de conjunto credal.

(a)

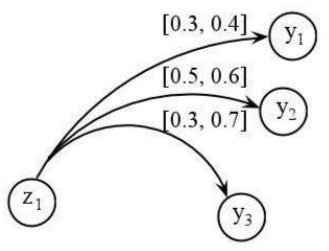

(b)

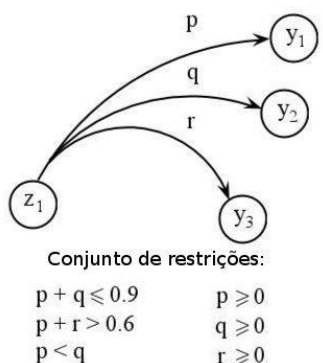

Figura 2.3: Duas maneiras de representar a imprecisão nas probabilidades de transição em um MDP.

Por exemplo, a Figura 2.3a tem como conjunto credal, todas as distribuições limitadas pelos intervalos das transições de estados. Na Figura 2.3b, o conjunto de distribuições de probabilidades que satisfazem o conjunto de restrições também define um conjunto credal.

Um conjunto credal que define as distribuições condicionais sobre o próximo estado $s^{\prime}$, dado um estado $s$ e a ação $a$ é referido como um conjunto credal de transição (transition credal set) e denotado por $K(s, a)$. Assumimos que todos os conjuntos credais são fechados e convexos (Cozman 2000; Walley 1991).

Definição 2.5 (MDP-IP). Formalmente um MDP-IP é definido por uma tupla $\langle S, A, R, K, \gamma\rangle$ em que (Delgado 2010):

- S é um conjunto discreto e finito de estados;

- A é um conjunto discreto e finito de ações;

- $R: S \times A \rightarrow \mathbb{R}$ é a função recompensa que associa a cada estado um valor real;

- $K(s, a)$ define um conjunto de medidas de probabilidade de transição válidas, i.e., um conjunto credal de transição. $K(s, a)$ pode ser implicitamente representado por um conjunto de probabilidades de transição consistente com um conjunto de restrições lineares.

- $\gamma$ é o fator de desconto.

Note que a definição de MDP-IP é a mesma dada para MDP, exceto que a função de transição $p\left(s^{\prime} \mid s, a\right)$ é substituída pelo conjunto de distribuições $K(s, a)$ (Delgado 2010). Assim, o MDPIP é um MDP em que as transições de estado são imprecisamente especificadas através dos conjuntos credais. O Exemplo 2.1 mostra que o conjunto credal $K(s, a)$ pode ser determinado por uma região bidimensional de todas as medidas de probabilidade que satisfazem o conjunto de restrições $C$.

Exemplo 2.1. Suponha que num MDP-IP seja dado um conjunto de estados $S=\left\{s_{0}, s_{1}, s_{2}\right\}$ e ações a $\in$ é modelada pelo conjunto de parâmetros de probabilidade $p\left(s^{\prime} \mid s, a\right) \in K(s, a)$, nomeados por $p_{0}, p_{1}$ e $p_{2}$, em que

$$
\begin{gathered}
p_{0}=p\left(s_{0} \mid s_{0}, a_{1}\right), \\
p_{1}=p\left(s_{1} \mid s_{0}, a_{1}\right), \\
p_{2}=p\left(s_{2} \mid s_{0}, a_{1}\right),
\end{gathered}
$$

e o conjunto de restrições:

$$
C=\left\{p_{0} \leq 2 / 3, \quad p_{2} \leq 2 / 3, \quad 2 p_{0} \geq p_{1} \quad e \quad p_{0}+p_{1}+p_{2}=1\right\} .
$$

A região bi-dimensional de todas as medidas de probabilidade que satisfazem $C$ é mostrada na região cinza da Figura 2.4, que corresponde ao conjunto credal para o exemplo dado. 


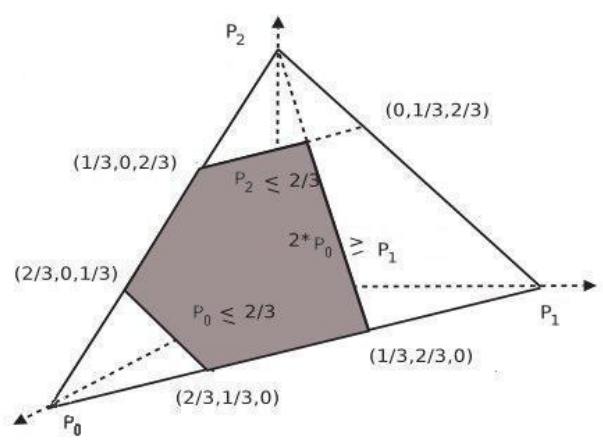

Figura 2.4: Exemplo de um conjunto credal definido pelos valores dos parâmetros de probabilidade $p_{0}, p_{1}$ e $p_{2}$ que satisfazem o conjunto de restrições $C$ (região cinza). Fonte: Cozman (2000)

Solução robusta para MDP-IP Há vários critérios de escolha para selecionar políticas em um MDPIP. O critério maximin seleciona uma política que produz a maior recompensa no pior caso das escolhas da Natureza (solução robusta), isto é:

$$
V^{*}(s)=\max _{a \in A}\left(R(s, a)+\gamma \min _{p \in K(s, a)} \sum_{s^{\prime} \in S} p\left(s^{\prime} \mid s, a\right) V^{*}\left(s^{\prime}\right)\right) .
$$

Apesar da solução de um MDP-IP ser mais complexa que a de um MDP, foram propostas soluções eficientes para MDP-IPs baseadas em Iteração de Valor e modelos fatorados (Delgado et al, 2009, 2011) que podem resolver problemas com até $2^{19}$ estados.

\subsubsection{Relações entre BMDPs, MDP-STs e MDP-IPs}

No trabalho de Trevizan et al. (2007) foi discutido o relacionamento entre MDPST, MDPIP e BMDP e foi mostrado que nem todo MDPST pode ser expresso como um BMDP, e vice versa. Além disso foi mostrado que o modelo BMDP e o modelo MDP-ST são casos especiais de MDP-IP (Trevizan et al, 2007). A Figura 2.5 mostra a relação entre esses três modelos de MDP com imprecisão e mostra também que todos são generalizações de MDP.

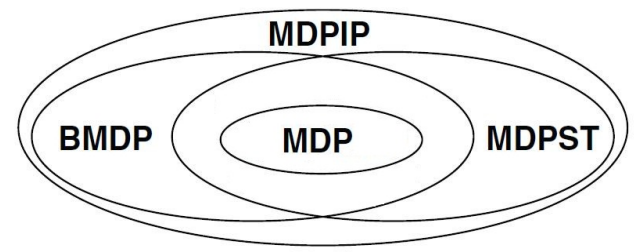

Figura 2.5: Relacionamentos entre modelos (BMDPs com recompensas precisas). Fonte: Trevizan (2006).

Nesse trabalho estendemos as ideias dessa seção e das seções anteriores de generalizações do MDP com imprecisão ou não determinismo na função de transição de estado para Jogos Markovianos Alternados ( $A l$ ternating Markov Games - AMGs). Na próxima seção (Seção 2.3) definimos o AMG com probabilidades precisas; no Capítulo 3 estabelecemos a correspondência entre MDP-STs e jogos markovianos alternados considerando a Natureza como um jogador adversário; e no Capítulo 4 definimos jogos markovianos alternados com probabilidades imprecisas (uma extensão de MDP-IPs para dois agentes).

\subsection{Jogo Markoviano Alternado - AMG}

Num jogo simultâneo de dois jogadores, dado um estado $s$, o Jogador I escolhe uma ação $a_{1}$ e o Jogador II escolhe uma ação $a_{2}$ e ambos os jogadores aplicam suas ações simultaneamente para que haja a transição do estado $s$ para $s^{\prime}$. Por outro lado, em jogos alternados a tomada de decisão é feita sequencialmente, ou seja, em um estado $s_{1}$ o Jogador I escolhe uma ação $a_{1}$ e após a ação ser executada, há uma transição do 
estado $s_{1}$ para um estado $s_{2}$ com probabilidade $p$. Em $s_{2}$ é a vez do Jogador II escolher uma ação $a_{2}$ que fará a transição de $s_{2}$ para $s_{1}^{\prime}$ com probabilidade $q$, e assim por diante.

Um AMG (Alternating Markov Game) é definido por um conjunto de estados $S$, dividido em dois subconjuntos, $S_{1}$ e $S_{2}$ e a função de transição $p$ é definida separadamente para cada um dos jogadores. Assim, $S_{1}$ é o conjunto de estados no qual o Jogador I faz a escolha de ação e $S_{2}$ é o conjunto de estados em que o Jogador II faz a escolha de ação, sendo $S=S_{1} \cup S_{2}$ e $S_{1} \cap S_{2}=\varnothing$. Dessa forma, em cada estado $s \in S$ somente um dos jogadores tem a escolha de ação. Para que os jogadores possam fazer seus movimentos alternadamente, a função de transição de estados é dada por: $p\left(s^{\prime} \mid s_{1}, a\right)=0 \forall s^{\prime}, s_{1} \in S_{1}$ e $a \in A_{1}$, caso contrário, se $s^{\prime} \in S_{2}$ então $0 \leq p\left(s^{\prime} \mid s_{1}, a\right) \leq 1$. Da mesma forma, $p\left(s^{\prime} \mid s_{2}, a\right)=0 \forall s^{\prime}, s_{2} \in S_{2}$ e $a \in A_{2}$, caso contrário, se $s^{\prime} \in S_{1}$ então $0 \leq p\left(s^{\prime} \mid s_{2}, a\right) \leq 1$. A função recompensa para o caso de AMG é $R_{1}\left(s_{1}\right)$ associada ao Jogador I no estado $s_{1} \in S_{1}$ e $R_{2}\left(s_{2}\right)$ é a associada ao Jogador II no estado $s_{2} \in S_{2}$.

Formalmente, podemos definir o modelo AMG de dois jogadores como:

Definição 2.6 (AMG). Um Jogo Markoviano Alternado de horizonte infinito é definido pela tupla $\left\langle S_{1}, S_{2}\right.$, $\left.A_{1}, A_{2}, p_{1}, p_{2}, R_{1}, R_{2}, \gamma\right\rangle$ em que:

- $S_{1}$ é um conjunto finito de estados do Jogador I;

- $S_{2}$ é um conjunto finito de estados do Jogador II;

- $A_{1}$ é o conjunto finito de ações disponíveis para o Jogador I;

- $A_{2}$ é o conjunto finito de ações disponíveis para o Jogador II;

- $p_{1}: S_{1} \times A_{1} \times S_{2} \rightarrow P D\left(S_{2}\right)$ é a função de transição de estado em que o Jogador I escolhe uma ação em um dado estado que o leva para um estado em $S_{2}$. $P D\left(S_{2}\right)$ representa o conjunto discreto de distribuição de probabilidades sobre $S_{2}$;

- $p_{2}: S_{2} \times A_{2} \times S_{1} \rightarrow P D\left(S_{1}\right)$ é a função de transição de estado em que o Jogador II escolhe uma ação em um dado estado que o leva para um estado em $S_{1} . P D\left(S_{1}\right)$ representa o conjunto discreto de distribuição de probabilidades sobre $S_{1}$;

- $R_{1}: S \times A_{1} \rightarrow \mathbb{R}$ é a função recompensa associada ao Jogador I;

- $R_{2}: S \times A_{2} \rightarrow \mathbb{R}$ é a função recompensa associada ao Jogador II;

- $\gamma$ é o fator de desconto da mesma forma como num $M G$.

Em jogos de dois jogadores de soma zero, um jogo é disputado entre jogadores com objetivos opostos, então a perda de um significa a vitória de outro. Dessa forma é possível representar as recompensas dos jogadores através de uma única função recompensa, a qual um jogador tenta maximizar e o outro jogador (oponente) tenta minimizar. Dessa forma, $R(s, a)$ é a recompensa imediata para o Jogador I quando este está no estado $s \in S_{1}$ e escolhe uma ação $a \in A_{1}$ ou a recompensa imediata para o Jogador I quando o Jogador II está no estado $s \in S_{2}$ e escolhe uma ação $a \in A_{2}$ (Littman 1996).

Chamamos de política estacionária uma política em que a ação especificada para cada estado independe do estágio do jogo. Definimos $\pi: S \rightarrow A_{1}(S)$ como a política estacionária para o Jogador I e por П o conjunto de todas as políticas estacionárias do Jogador I. Da mesma forma, $\Phi$ é o conjunto de todas as políticas estacionárias para o Jogador II.

O valor de um jogo que segue as políticas $\pi$ e $\phi$, isto é, as políticas dos Jogadores I e II, respectivamente, a partir de um estado inicial $s \in S$, é dado por (Littman 1996):

$$
V(\pi, \phi)\left(s_{1}\right)=R\left(s_{1}, \pi\left(s_{1}\right)\right)+\gamma \sum_{s_{2} \in S_{2}} p_{1}\left(s_{2} \mid s_{1}, \pi\left(s_{1}\right)\right) V(\pi, \phi)\left(s_{2}\right), \forall s_{1} \in S_{1}
$$

$\mathrm{e}$

$$
V(\pi, \phi)\left(s_{2}\right)=R\left(s_{2}, \phi\left(s_{1}\right)\right)+\gamma \sum_{s_{1} \in S_{1}} p_{2}\left(s_{1} \mid s_{2}, \phi\left(s_{2}\right)\right) V(\pi, \phi)\left(s_{1}\right), \forall s_{2} \in S_{2} .
$$


Note que a Equação (2.11) é a Equação de Bellman adaptada para o Jogador I, ou seja, calcula o valor do estado $s_{1}$ do Jogador I como a recompensa local mais a recompensa esperada futura considerando os valores dos estados do Jogador II. Similarmente, a Equação (2.12) é a Equação de Bellman do Jogador II.

\subsubsection{Políticas ótimas de equilíbrio para um AMG}

Em um MDP, uma política ótima é uma política que maximiza a soma esperada das recompensas descontadas e não é dominada, ou seja, não há nenhum estado no qual qualquer outra política pode obter uma recompensa esperada maior. Porém, para muitos jogos markovianos, não há uma política que seja não dominada porque o desempenho do Jogador I depende da escolha do Jogador II (Littman 1994). Então, como definir uma política ótima em um jogo markoviano? A ideia usada na literatura de teoria dos jogos para definir um comportamento ótimo para os jogadores é através da definição de equilíbrio de Nash.

Definição 2.7 (Políticas de equilíbrio). Um par de políticas $\pi^{*} \in \Pi e \phi^{*} \in \Phi$ é um par de políticas de equilíbrio se não existe uma política $\pi \in \Pi$ tal que (Chang 2006):

$$
V\left(\pi^{*}, \phi^{*}\right)(s)<V\left(\pi, \phi^{*}\right)(s), \quad s \in S,
$$

e não existe uma política $\phi \in \Phi$ tal que:

$$
V\left(\pi^{*}, \phi\right)(s)<V\left(\pi^{*}, \phi^{*}\right)(s), \quad s \in S .
$$

Isto é, as políticas $\pi^{*}$ e $\phi^{*}$ são escolhidas de forma que o Jogador I não possa receber $V(s)$ maior mudando suas ações e o Jogador II não possa receber $V(s)$ menor mudando suas ações.

Em alguns tipos de jogos markovianos pode haver mais de um par de políticas de equilíbrio e, em alguns casos, o valor pode não ser único. Entretanto, Shapley (1953) provou que todo jogo markoviano de dois jogadores de soma zero com probabilidades precisas possui um valor único, mesmo tendo mais de um par de políticas de equilíbrio. Assim, para os AMGs estudados nesse capítulo, a definição de equilíbrio coincide com a definição de uma política ótima usada em MDPs e, portanto, a solução para um AMG é encontrar um par de políticas ótimas de equilíbrio.

Uma forma de obter um par de políticas ótimas de equilíbrio em um AMG é encontrar, em cada estado do jogo, uma ação que seja a melhor para o Jogador I no pior caso de escolha de ação do Jogador II. Esta estratégia é conhecida na teoria dos jogos como maximin e, por ser pessimista, minimiza algo conhecido na teoria dos jogos como "regret" (Lavalle 2006). O regret pode ser visto como o arrependimento que um jogador tem por ter escolhido uma ação ruim e que desejaria tê-la trocado. Também pode ser visto como a quantidade de recompensa que poderia ser ganha escolhendo uma ação diferente.

\subsubsection{Encontrando pares de políticas ótimas de equilíbrio}

Para encontrar um par de políticas de equilíbrio em jogos markovianos alternados, pode-se usar o algoritmo de Iteração por Valor usado em MDP com algumas modificações. Como o desempenho do Jogador I depende da escolha do Jogador II, uma (solução robusta) para AMG de soma zero é encontrar uma política que seja a melhor para o Jogador I no pior caso de escolha de ação do Jogador II, para qualquer um dos estados. Assim, redefine-se $V^{*}(s)$ para ser a recompensa esperada para o Jogador I seguindo uma política minimax ótima contra um oponente com uma estratégia ótima. Dessa forma, o cálculo do valor ótimo de um estado $s \in S$ em um AMG de soma zero é dado por (Littman 1996):

$$
V^{*}\left(s_{1}\right)=\max _{a \in A_{1}}\left(R\left(s_{1}, a_{1}\right)+\gamma \sum_{s_{2} \in S_{2}} p_{1}\left(s_{2} \mid s_{1}, a\right) V^{*}\left(s_{2}\right)\right), \forall s_{1} \in S_{1}
$$

$\mathrm{e}$

$$
V^{*}\left(s_{2}\right)=\min _{a \in A_{2}}\left(R\left(s_{2}, a_{2}\right)+\gamma \sum_{s_{1} \in S_{1}} p_{2}\left(s_{1} \mid s_{2}, a\right) V^{*}\left(s_{1}\right)\right), \forall s_{2} \in S_{2} .
$$

Uma forma alternativa de descrever essa solução simplifica a maneira de calcular a função valor para 
AMG usando uma definição unificada da função de transição e função recompensa.

Definição 2.8 (AMG modelo unificado). Um jogo markoviano alternado de dois jogadores de soma zero é definido pela tupla $\left\langle S, A_{1}, A_{2}, P, R, \gamma\right\rangle$ de tal forma que:

- o conjunto de estados $S$ é decomposto em dois conjuntos, $S_{1}$ (estados do Jogador I) e $S_{2}$ (estados do Jogador II), em que $S_{1} \cap S_{2}=\varnothing$ e $S_{1} \cup S_{2}=S$;

- $A_{i}(s)$ é o conjunto finito de ações aplicáveis em s para o jogador $i$. Para cada jogador $i$, existe uma ação noop $\in A_{i}$ que pode ser executada em qualquer estado do jogo e cujo efeito é nulo, ou seja, se todos os agentes executarem suas ações noop no estado $s$, o jogo permanecerá no mesmo estado. Em cada estado $s \in\left(S_{1} \cup S_{2}\right)$ somente um jogador tem uma ou mais ações executáveis em $s$;

- a função de transição de estado é definida por $p: S \times A_{1} \times A_{2} \rightarrow P D(S)$ em que $P D(S)$ representa o conjunto de distribuições de probabilidades sobre $S$. Os jogadores fazem suas jogadas alternadamente dado que $p\left(s_{1}^{\prime} \mid s_{1}, a_{1}, a_{2}\right)=0$ para todos os $s_{1}, s_{1}^{\prime} \in S_{1}, a_{1} \in A_{1}\left(s_{1}\right), a_{2} \in A_{2}\left(s_{1}\right) e$ $p\left(s_{2}^{\prime} \mid s_{2}, a_{1}, a_{2}\right)=0$ para todos os $s_{2}, s_{2}^{\prime} \in S_{2}, a_{1} \in A_{1}\left(s_{2}\right), a_{2} \in A_{2}\left(s_{2}\right)$;

- a função de recompensa é dada por $R: S \times A_{1} \times A_{2} \rightarrow \mathbb{R}$ que representa as recompensas instantâneas do jogador. Como se trata de um jogo de soma zero, essa mesma função recompensa é positiva para o Jogador I e negativa para o Jogador II;

- $\gamma \in] 0,1[$ é o fator de desconto, como descrito anteriormente.

Assim, as Equações (2.11) e (2.12) que calculam o valor de um jogo que segue as políticas $\pi$ e $\phi$ podem ser unificadas numa única equação dada por:

$$
V(\pi, \phi)(s)=R(s, \pi(s), \phi(s))+\gamma \sum_{s^{\prime} \in S} p\left(s^{\prime} \mid s, \pi(s), \phi(s)\right) V(\pi, \phi)\left(s^{\prime}\right)
$$

e as Equações (2.13) e (2.14) podem ser unificadas resultando na solução ótima para AMGs com estados e função de transição dados pela Equação (2.16):

$$
V^{*}(s)=\max _{a_{1} \in A_{1}} \min _{a_{2} \in A_{2}}\left(R\left(s, a_{1}, a_{2}\right)+\gamma \sum_{s^{\prime} \in S} p\left(s^{\prime} \mid s, a_{1}, a_{2}\right) V^{*}\left(s^{\prime}\right)\right)
$$

As políticas ótimas, $\pi^{*}$ e $\phi^{*}$, são definidas calculando-se as funções arg max e $\arg$ min, respectivamente, ou seja:

$$
\left\langle\pi^{*}, \phi^{*}\right\rangle(s)=\arg \max _{a_{1} \in A_{1}} \min _{a_{2} \in A_{2}}\left(R\left(s, a_{1}, a_{2}\right)+\gamma \sum_{s^{\prime} \in S} p\left(s^{\prime} \mid s, a_{1}, a_{2}\right) V^{*}\left(s^{\prime}\right)\right) .
$$

$\mathrm{Na}$ Equação (2.16) a ordem dos cálculos de $\min$ e $\max$ não altera o valor do jogo. Esta propriedade foi provada por Shapley (1953), que também provou que a solução minimax (ou maximin) converge, isto é, a Equação (2.16) encontra o valor de equilíbrio de um jogo AMG de dois jogadores de soma zero.

Note que com a formulação da Equação (2.16) temos uma única função valor de equilíbrio, embora possam haver diferentes pares de políticas que satisfaçam a Equação (2.17).

O Algoritmo 3 (AMG-VALUE-ITERATION) implementa o algoritmo de Iteração de Valor para AMGs. Ele recebe como parâmetro de entrada o AMG dado pela tupla $\left\langle S, A_{1}, A_{2}, R, p, \gamma\right\rangle$ e o número máximo de iterações dado por maxIter e devolve a função valor ótima e o par de políticas de equilíbrio.

De forma análoga, o Algoritmo 4 (AMG-POLICY-ITERATION) implementa o algoritmo de Iteração de política para AMGs. Note que esse algoritmo recebe como parâmetro de entrada o AMG, faz chamadas aos Algoritmos 5, 6, 7 e 8 e devolve como resposta a função valor ótima e o par de políticas de equilíbrio. Esse algoritmo foi adaptado do algoritmo descrito em Littman (1996), Capítulo 4. 

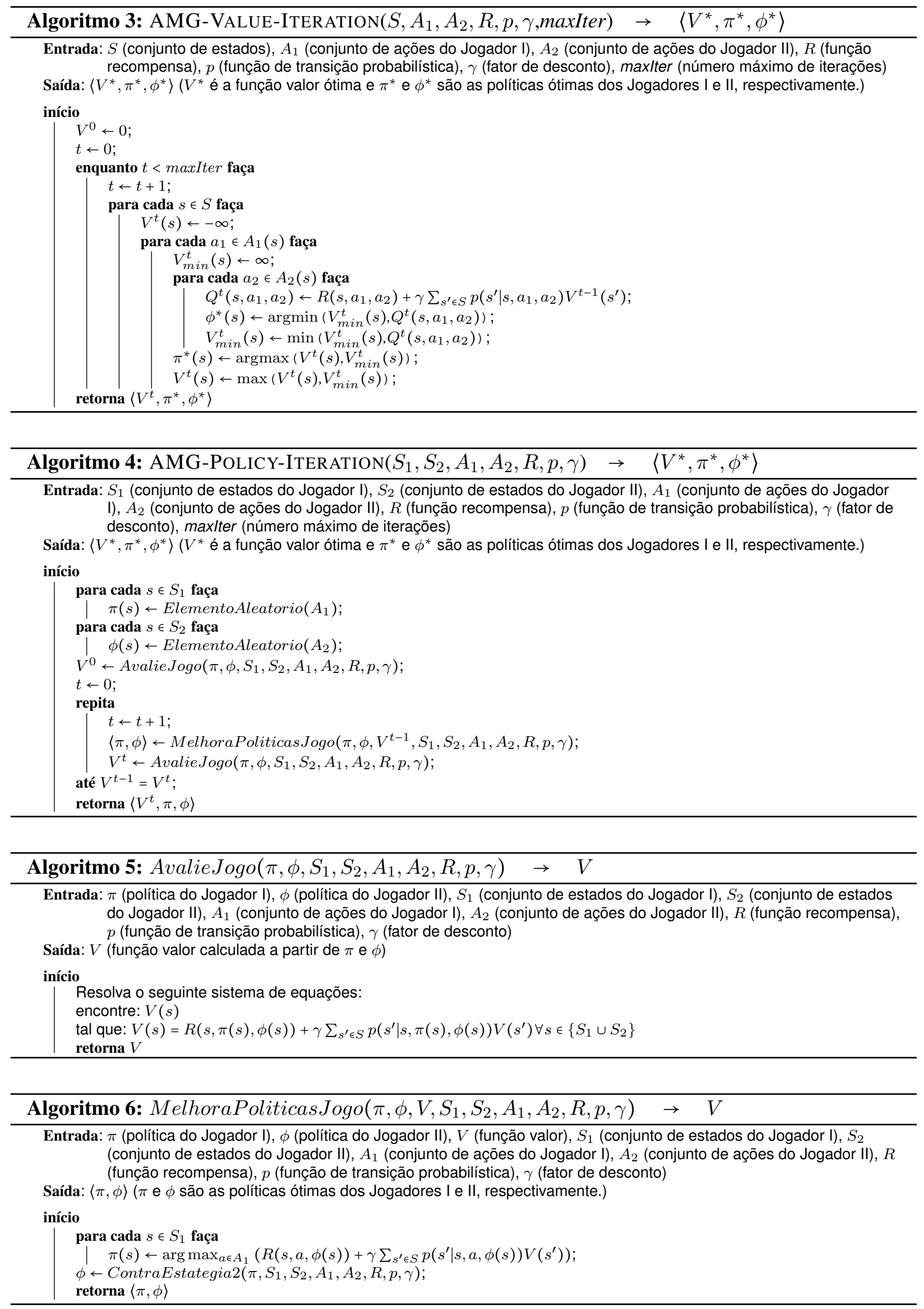

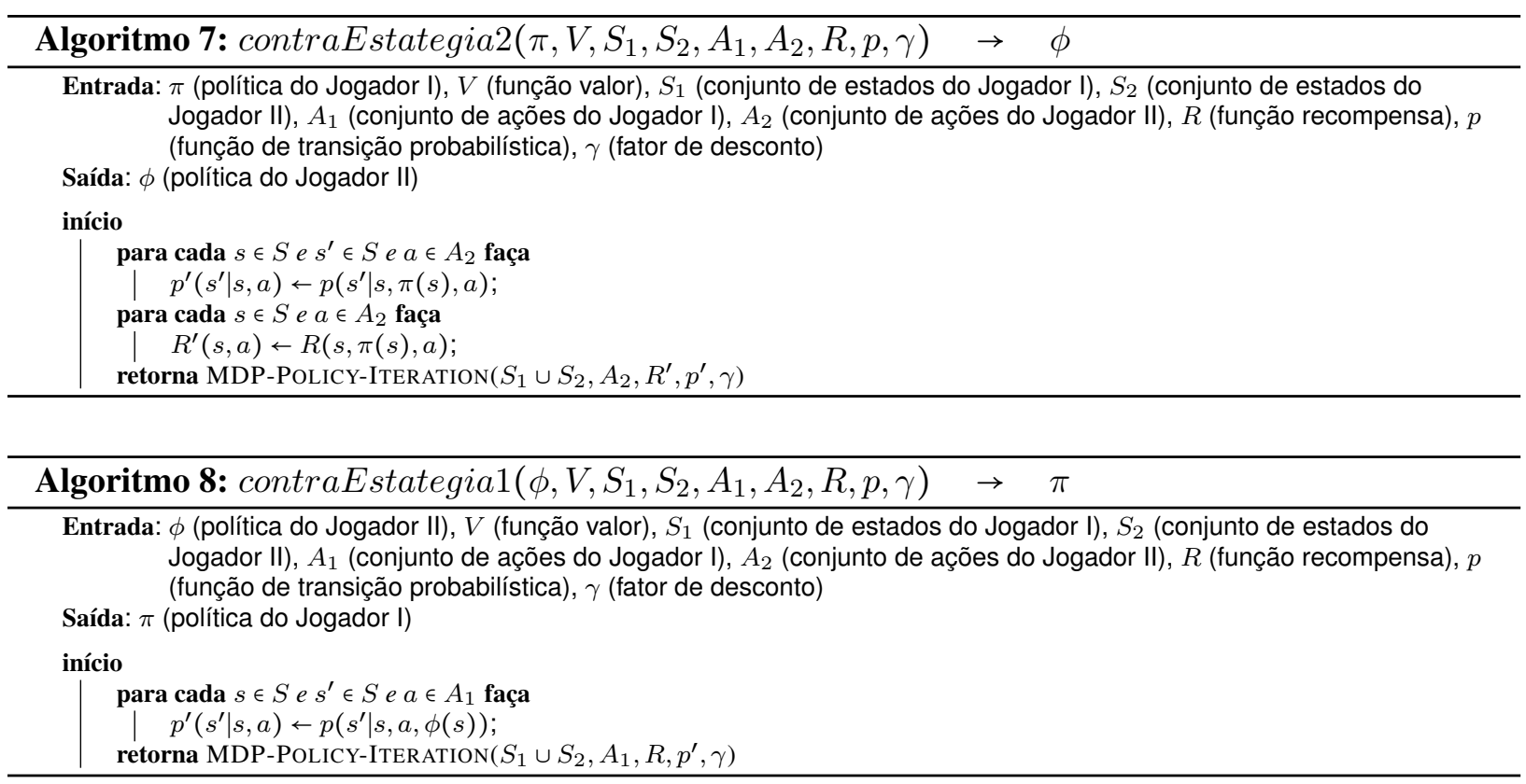

Note que em ambos os algoritmos (AMG-VALUE-ITERATION e AMG-PoliCY-ITERATION) a função valor ótima devolvida é um valor real para cada estado. Nos próximos capítulos resolvemos AMGs com imprecisões nas probabilidades de transição de estados e como resultado, a função valor calculada é dada por intervalos.

Segundo Littman (1994) os AMGs são um tipo de MDP generalizado e o problema de encontrar uma solução para um AMG está na classe de complexidade NP $\cap$ co-NP. Isto porque podemos "adivinhar" uma política para cada jogador e verificar a otimalidade em tempo polinomial usando um algoritmo para resolver o MDP resultante. 


\section{Capítulo 3}

\section{Jogos Markovianos Alternados com Transição Valorada por Con- junto}

Uma vez que o objetivo desse trabalho é investigar novos modelos e soluções para jogos markovianos alternados com diferentes formas de incerteza, neste capítulo discutimos a relação entre um AMG (Seção 2.3) e um MDP-ST (Seção 2.1). Mostramos que uma solução eficiente para MDP-STs pode ser derivada do modelo AMG correspondente em que um dos jogadores faz o papel da Natureza. Além disso, como uma consequência natural de tal modelagem, propomos um novo modelo de jogo markoviano alternado com incerteza na transição de estados. Esses estudos resultaram no trabalho descrito em de Barros et al. (2012) e serão discutidos em detalhes neste capítulo.

\subsection{Um jogo contra a Natureza}

Como foi discutido anteriormente, um MDP-ST pode ser visto como um jogo markoviano alternado de dois jogadores, em que o Jogador I é o jogador para o qual se deseja calcular a política ótima, e o Jogador II, faz o papel da Natureza.

Seja um MDP-ST= $\langle S, A, F, p, R, \gamma\rangle$ (como na Definição 2.4), em que: $S$ é um conjunto finito de estados; $A$ é um conjunto finito de ações; $p(k \mid s, a)$, com $s \in S, a \in A$ e $k \in 2^{S} \backslash \varnothing$, a função de transição probabilística valorada por conjunto; e $R(s, a)$ a função recompensa. Podemos caracterizar um AMG de dois jogadores de soma zero $\left\langle S_{1}, S_{2}, A_{1}, A_{2}, p_{1}, p_{2}, R, \gamma^{\prime}\right\rangle$ a partir do MDP-ST $\langle S, A, F, p, R, \gamma\rangle$ em que:

- $S_{1}=S$ é o conjunto finito de estados do Jogador I;

- $S_{2}=2^{S}$ é o conjunto finito de estados do Jogador II. Cada estado $k \in S_{2}$ representa um conjunto de estados alcançáveis em um passo;

- $A_{1}=A$ é o conjunto finito de ações do Jogador I com efeitos probabilísticos e aplicáveis aos estados $s \in S_{1}$;

- $A_{2}$ é o conjunto finito de ações do Jogador II com efeitos determinísticos e aplicáveis em $k \in S_{2}$. Essas ações são do tipo seleciona_ $s_{i}$ que quando aplicada a $k \in S_{2}$ leva o jogador ao estado $s_{i}$ com probabilidade 1 e com 0 para os demais;

- $F$ é a função que mapeia o estado e a ação para um conjunto de estados;

- $p_{1}=p$ é a função de transição probabilística de estado para conjuntos do Jogador I, isto é, para $k \in S_{2}$;

- $p_{2}: S_{2} \times A_{2} \times S_{1}$ é a função de transição determinística de estado para o Jogador II;

- $R$ é a função recompensa do Jogador I. Uma vez que no MDP-ST só é definida recompensa sobre os estados de $S$, para os conjuntos de estados alcançáveis $k_{i} \in 2^{S}$ e $a \in A_{2}$, definimos $R\left(k_{i}, a\right)=0$;

- $\gamma^{\prime 2}=\gamma$ para adotar a mesma taxa de desconto, já que no AMG a taxa é descontada duas vezes (Equações (2.13) e (2.14)).

Vamos ilustrar como o jogo pode ser obtido através do MDP-ST por meio do exemplo dado pela Figura 3.1. 


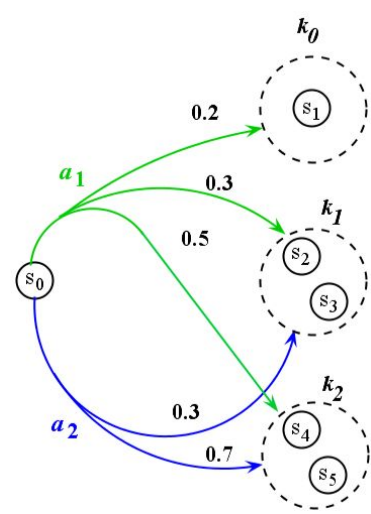

(a)

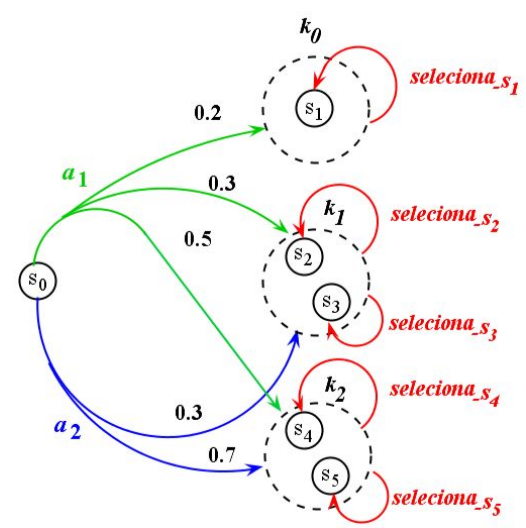

(b)

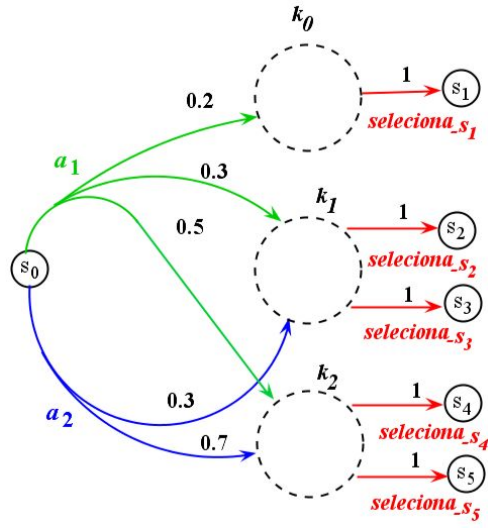

(c)

Figura 3.1: (a) MDP-ST. (b) o mesmo MDP-ST modelado por um AMG correspondente em que o Jogador II faz o papel da Natureza. (c) o mesmo AMG ilustrado em (b) mostrando explicitamente os estados alcançáveis $k_{i}$, em que $i \in\{0,1,2\}$.

A Figura 3.1(b) ilustra um jogo AMG construído a partir do MDP-ST da Figura 3.1(a). Nesse jogo, o Jogador I inicia em $s_{0}$, recebe uma recompensa e pode escolher executar uma das ações $a_{1}$ e $a_{2} \in A$, sabendo que: a ação $a_{1}$ o levará, com probabilidades $0.2,0.3$ e 0.5 , para os conjuntos de estados $k_{0}, k_{1}$, e $k_{2} \in 2^{S} \backslash \varnothing$, respectivamente; e que a ação $a_{2}$ o levará, com probabilidades 0.3 e 0.7 , para os conjuntos de estados $k_{1}$, e $k_{2} \in 2^{S} \backslash \varnothing$, respectivamente. Após a escolha de ação do Jogador I, é a vez do Jogador II (Natureza) fazer a sua escolha de ações.

O Jogador II, que atua no papel da Natureza, deve escolher uma ação para ser executada a partir de um conjunto de estados $k_{i} \in 2^{S} \backslash \varnothing$, sendo $k_{i}$, com $i \in\{0,1,2\}$, o resultado da ação previamente executada pelo Jogador I. Assim, as ações aplicáveis do Jogador II são definidas por $A(k)$ e possuem efeitos determinísticos, ou seja, a probabilidade de ir para o estado $s_{i}$ usando a ação seleciona_s $s_{i}$ é 1. Assim, o conjunto de ações possíveis da Natureza é dado pelas ações determinísticas $A_{2}=\left\{\right.$ seleciona_s $s_{0}$, seleciona_s $s_{1}, \ldots$, seleciona_s $\left.s_{5}\right\}$. Por exemplo, na Figura 3.1(b), a ação seleciona_s $s_{2}$, com probabilidade 1 leva o Jogador II ao estado $s_{2}$ e com probabilidade 0, para os demais estados $s_{i} \in k_{1}$, em que $i \in\{2,3\}$. A Figura 3.1(c) ilustra melhor como o MDP-ST da Figura 3.1(a) pode ser visto como um AMG com escolhas de ações determinísticas da Natureza.

Definição 3.1. Chamamos de $p_{N a t}: S_{2} \times A_{2} \times S \rightarrow P D(S)$, com $S_{2}=2^{S}$, a função de transição de estado da Natureza em que $A_{2}=\left\{\right.$ seleciona_s $s_{0}, \ldots$, seleciona_s $s_{i}, \ldots$, seleciona_s $\left.s_{n}\right\}, p\left(s \mid k\right.$, seleciona $\left.\_s_{i}\right)=1$ para $s=s_{i} \in k$ e 0 para $s \neq s_{i}$, com $k \in S_{2}=2^{S}$.

Teorema 3.1. Num AMG em que $p_{2}=p_{N a t}$ a política ótima do AMG para o Jogador I é igual à política ótima calculada para um MDP-ST correspondente.

Demonstração. Dadas as equações de um AMG:

$$
V^{*}\left(s_{1}\right)=\max _{a \in A_{1}}\left(R\left(s_{1}, a\right)+\gamma^{\prime} \sum_{s_{2} \in S_{2}} p_{1}\left(s_{2} \mid s_{1}, a\right) V^{*}\left(s_{2}\right)\right), \forall s_{1} \in S_{1}
$$

$e$

$$
V^{*}\left(s_{2}\right)=\min _{a \in A_{2}}\left(R\left(s_{2}, a\right)+\gamma^{\prime} \sum_{s_{1} \in S_{1}} p_{2}\left(s_{1} \mid s_{2}, a\right) V^{\star}\left(s_{1}\right)\right), \forall s_{2} \in S_{2} .
$$

Sendo o Jogador I o agente MDP-ST e o Jogador II a Natureza, podemos reescrever as Equações (3.1) e (3.2) da seguinte forma:

$$
V^{*}(s)=\max _{a \in A}\left(R(s, a)+\gamma^{\prime} \sum_{k \in F(s, a)} p(k \mid s, a) V^{*}(k)\right), \forall s \in S
$$


$e$

$$
V^{*}(k)=\min _{\text {seleciona_s } s_{i} \in k}\left(R(k, a)+\gamma^{\prime} V^{*}\left(s_{i}\right)\right), \forall k \in F(s, a) \text { e } s_{i} \in S .
$$

Sendo $R(k, a)=0$ e como as ações seleciona_s $s_{i}$ selecionam um estado $s_{i}$ dentro do conjunto $k$, temos a partir da Equação (3.4):

$$
V^{*}(k)=\min _{s_{i} \in k} \gamma^{\prime} V^{*}\left(s_{i}\right), \forall k \in F(s, a) \text { e } s_{i} \in S .
$$

Agora, substituindo (3.5) em (3.3) temos:

$$
V^{*}(s)=\max _{a \in A(s)}\left(R(s, a)+\gamma \sum_{k \in F(s, a)} p(k \mid s, a) \min _{s^{\prime} \in k} V^{*}\left(s^{\prime}\right)\right)
$$

que corresponde à Equação (2.9).

\subsection{Exemplo do robô vigilante}

Considere um cenário em que um robô vigilante deve permanecer num corredor de um prédio, sem atrapalhar a passagem das pessoas (Pereira e de Barros 2007). Como do lado esquerdo desse corredor existem portas, o robô atrapalha menos quando fica do lado direito. Assim, a cada instante, o robô deve decidir qual a melhor forma de agir: permanecer onde está ou se deslocar até outra posição. Porém, quando o robô escolhe uma ação, há uma incerteza sobre o resultado da ação aplicada: se o robô está no lado esquerdo do corredor (estado "esquerda") e escolhe ir para a direita (estado "direita"), com probabilidade 0.3 o robô permanece no mesmo lado, porém, com probabilidade 0.7 o robô pode ir (não-deterministicamente) para o meio ou para a direita do corredor.

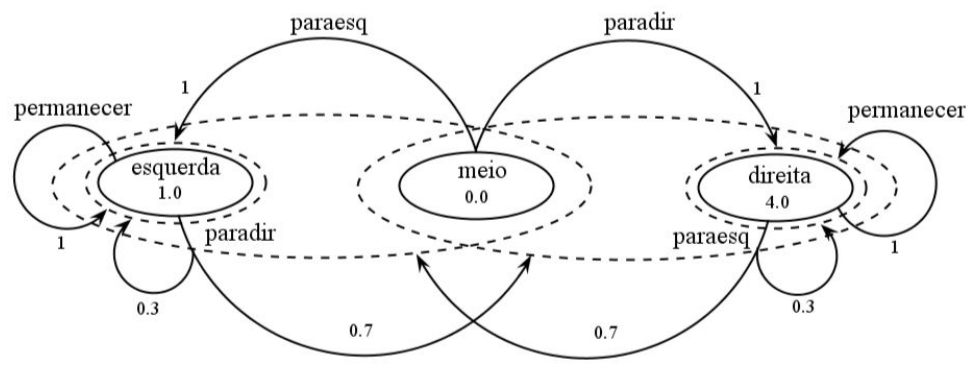

Figura 3.2: MDP-ST para exemplo do robô vigilante.

Esse problema pode ser modelado por meio de um MDP-ST ( $S, A, F, p, R, \gamma)$ (Figura 3.2), em que $S=\{$ esquerda, meio, direita $\}, A=\{$ permanecer, paradir, paraesq $\}$, $p$ é a função de transição (valores indicados nas transições), $R$ é a função recompensa (valores indicados dentro dos estados) e com $\gamma=0.9$.

Esse problema também pode ser modelado por um AMG de soma zero $\left(S_{1}, S_{2}, A_{1}, A_{2}, F, p_{1}, p_{2}\right.$, $R, \gamma)$, em que $S_{1}$ é o conjunto de estados do ambiente, ou seja, $S_{1}=\{$ esquerda, meio, direita $\}$ e $S_{2}$ é o conjunto de estados alcançáveis em um passo, ou seja, $S_{2}=\{$ esquerda, meio $\}$, \{direita, meio $\}$, $\{$ esquerda $\},\{$ direita $\}\} ; A_{1}=\{$ permanecer, paradir, paraesq $\} ; A_{2}=\{$ selecionaesq, selecionadir, selecionameio\}.

A Tabela 3.1 (coluna 1) mostra os resultados do cálculo de duas iterações da função valor (Equação (2.9)) para o MDP-ST da Figura 3.2. Na Tabela 3.1 (coluna 2) mostramos o resultado do cálculo da função valor do jogo AMG correspondente. Note que foram necessárias 4 iterações para se chegar aos mesmos valores encontrados para a função valor do MDP-ST nos estados $\left(s_{0}, s_{1}, s_{2}\right)$. Além disso, foi necessário também calcular os valores de $V$ para os estados alcançáveis $\left(k_{0}, k_{1}, k_{01}, k_{12}\right.$, em que $k_{i j}$ indica que o estado alcançável $k$ é composto pelos estados $s_{i}$ e $s_{j}$ ).

Com esse exemplo foi possível observar que as soluções para as duas formulações do problema, AMG com o Jogador II sendo a Natureza e MDP-ST são equivalentes, isto é, $V_{i}(s)$ é o mesmo nas duas abordagens. 


\begin{tabular}{|c|c|c|}
\hline iteração & cálculos efetuados - MDPST & cálculos efetuados - AMG \\
\hline 0 & $\begin{array}{l}V_{0}\left(s_{0}\right)=R\left(s_{0}\right)=1 \\
V_{0}\left(s_{1}\right)=R\left(s_{1}\right)=0 \\
V_{0}\left(s_{2}\right)=R\left(s_{2}\right)=4\end{array}$ & $\begin{array}{l}V_{0}\left(s_{0}\right)=R\left(s_{0}\right)=1 \\
V_{0}\left(s_{1}\right)=R\left(s_{1}\right)=0 \\
V_{0}\left(s_{2}\right)=R\left(s_{2}\right)=4 \\
V_{0}\left(k_{0}\right)=R\left(k_{0}\right)=0 \\
V_{0}\left(k_{2}\right)=R\left(k_{2}\right)=0 \\
V_{0}\left(k_{01}\right)=R\left(k_{01}\right)=0 \\
V_{0}\left(k_{12}\right)=R\left(k_{12}\right)=0\end{array}$ \\
\hline 1 & $\begin{array}{l}V_{1}\left(s_{0}\right)=1.9 \\
V_{1}\left(s_{1}\right)=3.6 \\
V_{1}\left(s_{2}\right)=7.6\end{array}$ & $\begin{array}{l}V_{1}\left(s_{0}\right)=1 \\
V_{1}\left(s_{1}\right)=0 \\
V_{1}\left(s_{2}\right)=4 \\
V_{1}\left(k_{0}\right)=1 \\
V_{1}\left(k_{2}\right)=4 \\
V_{1}\left(k_{01}\right)=0 \\
V_{1}\left(k_{12}\right)=0\end{array}$ \\
\hline 2 & $\begin{array}{l}V_{2}\left(s_{0}\right)=3.781 \\
V_{2}\left(s_{1}\right)=6.84 \\
V_{2}\left(s_{2}\right)=10.84\end{array}$ & $\begin{array}{l}V_{2}\left(s_{0}\right)=1.9 \\
V_{2}\left(s_{1}\right)=3.6 \\
V_{2}\left(s_{2}\right)=7.6 \\
V_{2}\left(k_{0}\right)=1 \\
V_{2}\left(k_{2}\right)=4 \\
V_{2}\left(k_{01}\right)=0 \\
V_{2}\left(k_{12}\right)=0\end{array}$ \\
\hline 3 & & $\begin{array}{l}V_{3}\left(s_{0}\right)=1.9 \\
V_{3}\left(s_{1}\right)=3.6 \\
V_{3}\left(s_{2}\right)=7.6 \\
V_{3}\left(k_{0}\right)=1.9 \\
V_{3}\left(k_{2}\right)=7.6 \\
V_{3}\left(k_{01}\right)=1.9 \\
V_{3}\left(k_{12}\right)=3.6\end{array}$ \\
\hline 4 & & $\begin{array}{l}V_{4}\left(s_{0}\right)=3.781 \\
V_{4}\left(s_{1}\right)=6.84 \\
V_{4}\left(s_{2}\right)=10.84 \\
V_{4}\left(k_{0}\right)=1.9 \\
V_{4}\left(k_{2}\right)=7.6 \\
V_{4}\left(k_{01}\right)=1.9 \\
V_{4}\left(k_{12}\right)=3.6\end{array}$ \\
\hline
\end{tabular}

Tabela 3.1: Comparação entre o cálculo da função valor para o problema do robô vigilante modelado como um MDP-ST e como um AMG

\subsection{Jogo Markoviano Alternado com Transição Valorada por Conjunto para o Jogador I - AMG-ST-1}

A caracterização de um MDP-ST como um jogo AMG de soma zero resulta numa classe especial de AMG, em que o Jogador I probabilisticamente vai de um estado para um conjunto de estados alcançáveis $k$, enquanto o Jogador II escolhe estados de $k$. Esse modelo pode ser estendido considerando que a função de transição do Jogador II ao invés de determinística, também pode ser probabilística. Chamamos esse novo modelo de AMG-ST-1 (Alternating Markov Game with Set-valued Transition for player 1), em que consideramos o Jogador I como aquele que se deseja calcular a política ótima, e o Jogador II como um agente adversário (não completamente modelado), responsável pela imprecisão dos efeitos probabilísticos das ações do Jogador I. Assim, a tupla que define esse novo modelo é dada por $\left\langle S, A, R, F, p_{1}, p_{2}, \gamma\right\rangle$ e a função valor ótima é dada pelas equações:

$$
V^{*}(s)=\max _{a \in A_{1}}\left(R(s, a)+\gamma \sum_{k \in F(s, a)} p_{1}(k \mid s, a) V^{*}(k)\right), \forall s \in S
$$

$\mathrm{e}$

$$
V^{*}(k)=\min _{a \in A_{2}}\left(R(k, a)+\gamma \sum_{s \in S} p_{2}(s \mid k, a) V^{*}(s)\right), \forall k \in F(s, a) .
$$

A Figura 3.3 modela um exemplo de jogo AMG-ST-1 no domínio de futebol. Dados dois jogadores em que o Jogador I pode executar a ação chute a gol a partir do estado $s_{1}$ (bola na grande área) e o Jogador II pode executar as ações contra-ataque e defesa. Com probabilidade 0.4 o chute executado pelo Jogador I pode levá-lo a dois estados possíveis: bola roubada ou gol; e, com probabilidade 0.6 para o estado gol ou escanteio. O Jogador II executará a ação contra-ataque quando há possibilidade de bola roubada e, com 
probabilidade 0.6 , roubará a bola $\left(s_{2}\right)$ ou, com probabilidade 0.4 , não conseguirá pegar a bola e acabará sofrendo o gol $\left(s_{3}\right)$. Por outro lado, se o chute executado pelo Jogador I apresentar uma situação de maior chance de gol, o Jogador II executará a ação defesa e, com probabilidade 0.5, colocará a bola em escanteio $\left(s_{4}\right)$, ou seja, realizará uma defesa que apenas tirará a bola da trajetória do gol colocando-a para fora, e com probabilidade 0.5 não conseguirá defender e sofrerá o gol $\left(s_{3}\right)$. É evidente que o estado $s_{3}$ (gol) é o estado que trará a maior recompensa para o Jogador I e por esse motivo o Jogador II tentará evitar que o Jogador I consiga chegar neste estado.

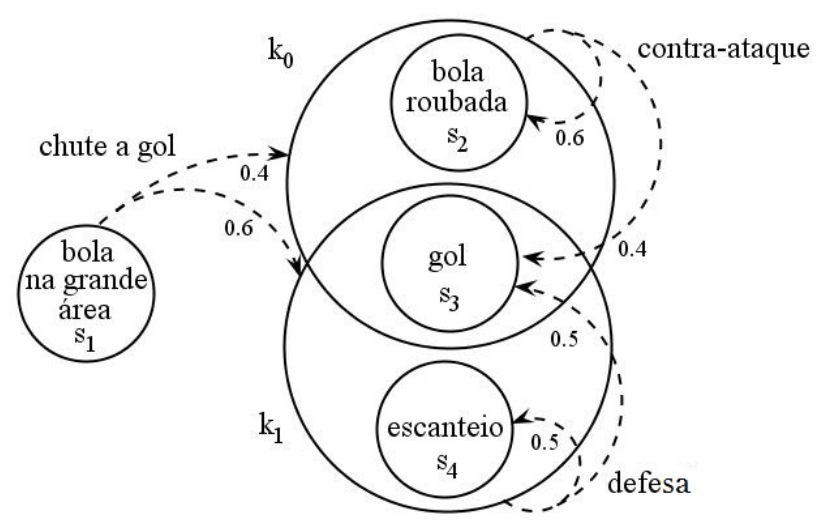

Figura 3.3: Um jogo de futebol modelado por um AMG com transições para conjuntos de estados para o Jogador I (AMG-ST-1).

Note que com essa maneira de modelar o jogo, os estados intermediários (por exemplo, a situação em que a bola está no ar) são implicitamente representados pelos conjuntos de estados $k_{0}$ e $k_{1}$. Por exemplo, a habilidade do Jogador I de executar a ação chute a gol, é representada pela distribuição de probabilidade para os conjuntos $k_{0}$ e $k_{1}$ : com probabilidade 0.4 o jogador tem a bola roubada ou gol e com 0.6 a bola é lançada para escanteio ou para o gol. 


\section{Capítulo 4}

\section{Jogos Markovianos Alternados com Probabilidades Imprecisas}

No capítulo anterior propomos um novo tipo de AMG com transições imprecisas, que chamamos de AMG-ST-I, que leva o Jogador I para um conjunto de estados $k$ e, em seguida, o Jogador II seleciona um estado $s$ desse conjunto, isto é, $s \in k$ (de Barros et al, 2012). Nesse capítulo apresentamos outro tipo de incerteza em jogos markovianos alternados: um jogo em que a imprecisão na função de transição de estados dos dois jogadores é dada por conjuntos credais (Cozman 2000) (como nos MDP-IPs mostrados na Seção 2.2) e no qual não modelamos a Natureza como um dos jogadores (Franco et al, 2011).

Definição 4.1 (AMG-IP - modelo unificado). Um jogo markoviano alternado de dois jogadores de soma zero com probabilidades imprecisas (Alternating Markov Game with Imprecise Probabilities - AMG-IP) é definido pela tupla $\left\langle S, A_{1}, A_{2}, K, R, \gamma\right\rangle$, em que:

- $S$ é um conjunto discreto e finito de estados completamente observáveis do jogo. $S$ é composto de dois subconjuntos disjuntos $S_{1}$ (estados do Jogador I) e $S_{2}$ (estados do Jogador II), em que $S_{1} \cap S_{2}=\varnothing$ e $S_{1} \cup S_{2}=S$;

- $A_{1}$ é o conjunto finito de ações disponíveis para o Jogador I somente aplicáveis em $S_{1}$ (não aplicáveis em $S_{2}$ ). Existe uma ação noop $\in A_{1}$, com efeito nulo, que pode ser executada em todo estado $s \in S$ com efeito nulo;

- $A_{2}$ é o conjunto finito de ações disponíveis para o Jogador II somente aplicáveis em $S_{2}$ (não aplicáveis em $S_{1}$ ). Existe uma ação noop $\in A_{2}$, com efeito nulo, que pode ser executada em todo estado $s \in S$ com efeito nulo;

- $K: S \times A_{1} \times A_{2} \rightarrow 2^{P D(S)}$ é a função de transição de estado em que $2^{P D(S)}$ representa os subconjuntos do conjunto de todas as distribuições de probabilidades sobre $S$. Assim, $K\left(s, a_{1}, a_{2}\right)$ com $a_{1} \in A_{1}$ e $a_{2} \in A_{2}$ define um conjunto de medidas de probabilidade de transição, isto é, um conjunto credal de transição de s para $s^{\prime}$, executando $a_{1} \in A_{1}$ e $a_{2} \in A_{2} ; K\left(s, a_{1}, a_{2}\right)$ pode ser implicitamente representado por um conjunto de parâmetros $\vec{p}$ e um conjunto de restrições lineares sobre $\vec{p}$;

- $R: S \times A_{1} \times A_{2} \rightarrow \mathbb{R}$ é a função recompensa. Como se trata de um jogo de soma zero, essa função é positiva para o Jogador I e negativa para o Jogador II;

- $\gamma \in] 0,1[$ é o fator de desconto.

A Definição 4.1 é baseada na Definição 2.8 para AMGs com modelo unificado dada no Capítulo 2.1. Note que com a introdução de ações noop para a especificação do modelo unificado, os jogadores continuam fazendo suas jogadas alternadamente, isto é, em cada estado $s \in\left(S_{1} \cup S_{2}\right)$ o jogador $i$ executa a ação $a_{i} \in A_{i}$, com $a_{i} \neq$ noop , enquanto o outro jogador executa a ação noop. Como vimos na Seção 2.3.2, esse modelo unificado permite a especificação de soluções mais compactas. Como se trata de um jogo de soma zero, no AMG-IP o objetivo do Jogador I é maximizar sua recompensa acumulada enquanto que o objetivo do Jogador II é minimizar a recompensa acumulada do Jogador I.

A Figura 4.1a mostra um jogo em que o efeito de uma ação executada em $s_{1}$ leva o Jogador I para os estados $s_{2}, s_{6}$ e $s_{4}$. O conjunto de distribuições de probabilidades que satisfazem as restrições dos intervalos 
dessa transição de estados define um conjunto credal. Chamamos esse modelo de AMG-INTERVAL. No entanto, o uso de conjuntos credais pode ser ainda uma forma mais geral de definir o conjunto de distribuições que satisfazem restrições na forma de intervalos e outros tipos de restrições. Por exemplo, a Figura 4.1b mostra que um conjunto credal pode ser definido a partir de um conjunto de restrições sobre os parâmetros $p, q$ e $r$ que representam as probabilidades de transição de um jogo AMG-IP.
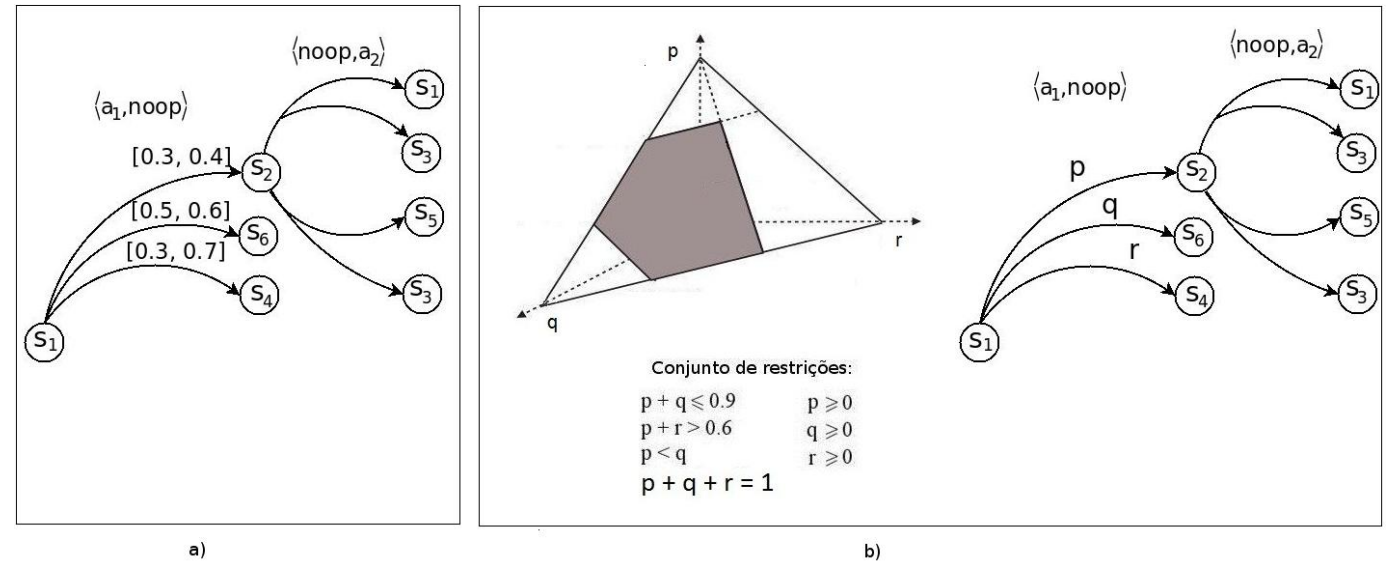

Figura 4.1: Escolhas de ações dos jogadores I e II em cada estágio T. (a) AMG-IP com probabilidades imprecisas dadas por intervalos (AMG-INTERVAL); (b) AMG-IP com probabilidades imprecisas dadas por restrições quaisquer sobre os parâmetros $p$, q e r. O conjunto credal é definido pelos valores de parâmetros de probabilidade $p$, q e r que satisfazem o conjunto de restrições (região cinza).

AMG-IP: Modelo Conceitual Num AMG-IP, o Jogador I seleciona e executa uma ação $a_{1}$ num estado $s$ e o Jogador II executa a ação noop. O jogo faz uma transição com uma escolha (da Natureza) de uma distribuição de probabilidades pertencente ao conjunto $K\left(s, a_{1}\right.$, noop $)$. No estado $s^{\prime}$ resultante da execução do par de ações $\left\langle a_{1}, n o o p\right\rangle$, o Jogador II faz a sua escolha e executa uma ação $a_{2}$ e o Jogador I executa a ação noop, seguido novamente por uma escolha da Natureza de uma distribuição de probabilidades em $K\left(s\right.$, noop,$\left.a_{2}\right)$. A cada par de ações executadas, os dois jogadores recebem uma recompensa (de soma zero).

Chang (2006) propôs um modelo AMG de dois jogadores de soma zero com a imprecisão nas probabilidades dada por intervalos (AMG-INTERVAL). Assim como num BMDP (Seção 2.2.1), esse jogo faz uma transição com uma escolha de probabilidades $p\left(. \mid s, a_{1}, a_{2}\right) \in d\left(s, a_{1}, a_{2}\right)$, em que $d\left(s, a_{1}, a_{2}\right)=$ $\left\langle\underline{p}\left(. \mid s, a_{1}, a_{2}\right), \bar{p}\left(. \mid s, a_{1}, a_{2}\right)\right\rangle$ é um intervalo de distribuições de probabilidade associado ao estado $s$ aplicando as ações $a_{1}$ e $a_{2}$. O AMG-IP é mais geral que o modelo AMG-INTERVAL proposto por Chang (2006). Nesse capítulo estendemos o trabalho de Chang (2006), propondo novas soluções que podem ser consideradas melhores, em geral.

\subsection{Políticas de equilíbrio para um AMG-IP}

Seja um conjunto $\Pi$ de políticas estacionárias para o Jogador I e um conjunto $\Phi$ de políticas estacionárias para o Jogador II. Seja um par de políticas $\pi \in \Pi$ e $\phi \in \Phi$. Com base na Equação (2.15) para o valor de um par de políticas de um AMG e considerando a função de transição de estados dada pelo conjunto credal $K(s, \pi(s), \phi(s))$, temos:

$$
V(\pi, \phi)(s)=R(s, \pi(s), \phi(s))+\gamma \sum_{s^{\prime} \in S} p\left(s^{\prime} \mid s, \pi(s), \phi(s)\right) V(\pi, \phi)\left(s^{\prime}\right), \quad p \in K(s, \pi(s), \phi(s)) .
$$

Note que o valor de $V(\pi, \phi)(s)$ depende da distribuição de probabilidade escolhida pela Natureza, $p \in K(s, \pi(s), \phi(s))$.

Com base na solução de MDP-IPs, o melhor que podemos fazer é definir um limite inferior para os valores de $V(\pi, \phi)(s)$. Uma vez que assumimos que o conjunto credal é fechado e convexo, sempre é possível obter um limite mínimo das distribuições de probabilidade (Walley 1991), isto é, $p \in K(s, \pi(s), \phi(s))$ que 
minimize a somatória:

$$
\underline{p}=p_{\min }(. \mid s, \pi(s), \phi(s)) \in \arg \min _{p \in K(s, \pi(s), \phi(s))} \sum_{s^{\prime} \in S} p\left(s^{\prime} \mid s, \pi(s), \phi(s)\right) \underline{V}(\pi, \phi)(s),
$$

e $\operatorname{assim} V(\pi, \phi)(s)=\underline{V}(\pi, \phi)(s)$ fazendo:

$$
\underline{V}(\pi, \phi)(s)=R(s, \pi(s), \phi(s))+\gamma \min _{p \in K(s, \pi(s), \phi(s))} \sum_{s^{\prime} \in S} p\left(s^{\prime} \mid s, \pi(s), \phi(s)\right) \underline{V}(\pi, \phi)\left(s^{\prime}\right),
$$

ou seja, dadas as políticas $\pi$ e $\phi$ dos dois jogadores, a distribuição de probabilidades $p \in K(s, \pi(s), \phi(s))$ é selecionada pela Natureza de forma a minimizar a recompensa esperada nos estágios futuros. Para realizar essa minimização considerando o caso mais geral de probabilidades imprecisas dadas por um conjunto qualquer de restrições sobre as transições de estados (Figura 4.1), é preciso fazer chamadas a um NLP-Solver (programa de otimização não linear, por exemplo, Minos (disponível em: http://www.ampl.com/index.html), o que torna os problemas AMG-IPs mais complexos que os AMGs descritos na Seção 2.3. Assim, com base na Equação (4.3) e na definição de políticas de equilíbrio para AMGs (Seção 2.3.2), podemos definir uma solução robusta para AMG-IPs que calcula o valor ótimo maximin para as piores escolhas da Natureza (min):

$$
\underline{V}^{*}(s)=\max _{a_{1} \in A_{1}} \min _{a_{2} \in A_{2}}\left(R\left(s, a_{1}, a_{2}\right)+\gamma \min _{p \in K\left(s, a_{1}, a_{2}\right)} \sum_{s^{\prime} \in S} p\left(s^{\prime} \mid s, a_{1}, a_{2}\right) \underline{V}^{*}\left(s^{\prime}\right)\right) .
$$

Chang (2006) mostrou que essa solução converge depois de $n$ iterações e, assim, é possível mostrar que os pares de políticas que alcançam $\underline{V}^{*}(s)$ são pares de políticas de equilíbrio $\langle\Pi, \Phi\rangle$ para o AMG com probabilidades imprecisas dadas por intervalos. Esse resultado também é verdadeiro para o caso mais geral de AMG-IPs, fazendo a suposição de conjuntos credais convexos e fechados (Cozman 2000; Walley 1991). No entanto, note que esse equilíbrio é apenas com relação à $V^{*}(s)$ (limite inferior da função valor que assume uma Natureza adversarial escolhendo $p \in K\left(s, a_{1}, a_{2}\right)$ que minimiza $\left.V(s)\right)$.

Apesar da solução (4.4) ser uma solução robusta, similar àquela proposta para MDP-IPs e suas variações (Seção 2.2), é possível melhorá-la considerando outras escolhas da Natureza (e assim definindo outro tipo de equilíbrio para o jogo). Da mesma forma que calculamos o limite inferior da função valor seguindo um par de políticas $\pi$ e $\phi$ (Equação (4.3)), também podemos calcular o seu limite superior. Novamente, como assumimos que o conjunto credal $K(s, \pi(s), \phi(s))$ é fechado e convexo, podemos calcular:

$$
\bar{p}=p_{\max }(. \mid s, \pi(s), \phi(s)) \in \arg \max _{p \in K(s, \pi(s), \phi(s)} \sum_{s^{\prime} \in S} p\left(s^{\prime} \mid s, \pi(s), \phi(s)\right) \bar{V}(\pi, \phi)(s)
$$

e $\operatorname{assim} V(\pi, \phi)(s)=\bar{V}(\pi, \phi)(s)$ fazendo:

$$
\bar{V}(\pi, \phi)(s)=R(s, \pi(s), \phi(s))+\gamma \max _{p \in K(s, \pi(s), \phi(s))} \sum_{s^{\prime} \in S} p\left(s^{\prime} \mid s, \pi(s), \phi(s)\right) \bar{V}(\pi, \phi)\left(s^{\prime}\right),
$$

ou seja, dado o par de políticas $\pi$ e $\phi, p \in K(s, \pi(s), \phi(s))$ é selecionado pela Natureza de forma a maximizar a recompensa esperada nos estágios futuros. Essa maximização também deve ser feita através de chamadas a um NLP-Solver. Assim, o valor ótimo maximin para as melhores escolhas da Natureza (max) é dado por:

$$
\bar{V}^{*}(s)=\max _{a_{1} \in A_{1}} \min _{a_{2} \in A_{2}}\left(R\left(s, a_{1}, a_{2}\right)+\gamma \max _{p \in K\left(s, a_{1}, a_{2}\right)} \sum_{s^{\prime} \in S} p\left(s^{\prime} \mid s, a_{1}, a_{2}\right) \bar{V}^{*}\left(s^{\prime}\right)\right),
$$


que corresponde a uma solução otimista para AMG-IPs.

É possível combinarmos as soluções dadas pelas Equações (4.4) e (4.7) com a seguinte ideia:

"Dentre os pares de políticas ótimas de equilíbrio $\langle\Pi, \Phi\rangle$ que satisfazem $\underline{V}^{*}(s)$ (isto é, supondo a Natureza adversarial), escolhemos um par de políticas de equilíbrio $\left\langle\pi^{*}, \phi^{*}\right\rangle$, com e $\phi^{*} \in \Phi$, que satisfazem $\bar{V}^{*}(s)$ (isto é, assumindo agora a Natureza cooperativa)".

Chamamos essa solução de Lower-First, porque ela primeiro calcula o limite inferior da função valor para depois calcular o limite superior. Em Chang (2006) foi proposta uma solução para AMGs com probabilidades imprecisas dadas por intervalos que inverte essa ordem:

"Dentre os pares de políticas ótimas de equilíbrio $\langle\Pi, \Phi\rangle$ que satisfazem $\bar{V}^{*}(s)$ (isto é, supondo a Natureza cooperativa), escolhemos um par de políticas de equilíbrio $\left\langle\pi^{*}, \phi^{*}\right\rangle$, com e $\phi^{*} \in \Phi$, que satisfazem $\underline{V}^{*}(s)$ (isto é, assumindo agora a Natureza adversarial)".

Chamamos essa solução de Upper-First, porque ela primeiro calcula o limite superior da função valor para depois calcular o limite inferior.

Apesar dessas duas novas soluções gerarem pares de políticas de equilíbrio aparentemente muito diferentes, nesse capítulo mostraremos situações em que elas podem devolver os mesmos pares de políticas de equilíbrio e situações em que qualquer uma pode encontrar uma política "melhor" que a outra. Além disso, propomos extensões que melhoram essas soluções em termos da recompensa esperada acumulada.

Para fazermos essa comparação, na próxima seção, definimos a função valor intervalar de um AMG-IP.

\subsubsection{Função Valor Intervalar}

As Equações (4.4) e (4.7) calculam o limite inferior e superior para a função valor ótima de um AMG-IP. Assim, dado um AMG-IP, definimos a função valor intervalar ótima dada por:

$$
\left[\underline{V}^{*}(s), \bar{V}^{*}(s)\right] .
$$

Note que as ações encontradas para calcular $\underline{V}^{*}(s)$ não são, necessariamente, as mesmas encontradas quando calculamos $\bar{V}^{*}(s)$, dado que esses cálculos são realizados de maneira independente. A Figura 4.2 ilustra uma situação em que dados dois pares de políticas $\left\langle\pi_{1}, \phi_{1}\right\rangle$ e $\left\langle\pi_{2}, \phi_{2}\right\rangle$, a função valor para o par $\left\langle\pi_{1}, \phi_{1}\right\rangle$ coincide apenas em seu limite inferior com $\underline{V}^{*}(s)$, isto é, $\underline{V}^{*}(s)=\underline{V}\left(\pi_{1}, \phi_{1}\right)(s)$, enquanto a função valor para o par $\left\langle\pi_{2}, \phi_{2}\right\rangle$ coincide apenas no limite superior com $\bar{V}^{*}(s)$, isto é, $\bar{V}^{*}(s)=\bar{V}\left(\pi_{2}, \phi_{2}\right)(s)$. Eventualmente, pode existir um par de políticas ótimas $\left\langle\pi^{*}, \phi^{*}\right\rangle$ cuja função valor intervalar seja exatamente $\left[\underline{V}^{*}(s), \bar{V}^{*}(s)\right]$. Porém, em geral, esse par não existe. No entanto, dado um par de políticas $\langle\pi, \phi\rangle$ podemos calcular a função valor do jogo seguindo essas políticas como sendo o intervalo:

$$
[\underline{V}(\pi, \phi)(s), \bar{V}(\pi, \phi)(s)],
$$

em que $\underline{V}(\pi, \phi)(s)$ é o valor do estado $s$ assumindo a Natureza adversária e $\bar{V}(\pi, \phi)(s)$ é o valor de $s$ assumindo a Natureza cooperativa, seguindo as políticas $\pi$ e $\phi$ (Equações (4.3) e (4.6)).

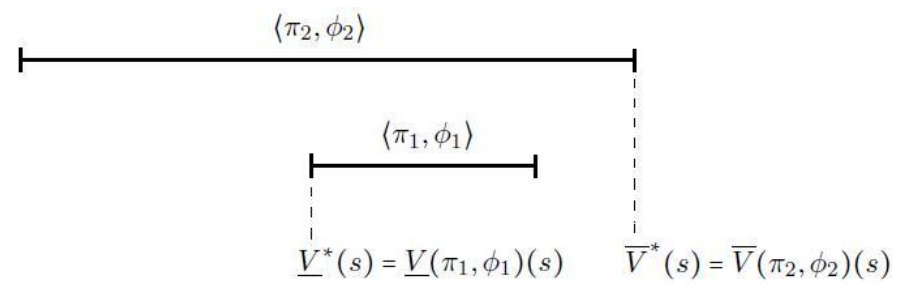

Figura 4.2: Os valores ótimos $\underline{V}^{*}(s) e \bar{V}^{*}(s)$ calculados pelas Equações (4.4) e (4.7) podem não corresponder aos valores de um único par de políticas ótimas. 
A questão então que queremos responder é: "Como encontrar um par de políticas ótimas $\pi^{*} e \phi^{*}$ que faça o melhor compromisso entre $\underline{V}^{*}(s) e \bar{V}^{*}(s)$ ?". Em outras palavras, como encontrar $\pi^{*}$ e $\phi^{*}$ que faça o melhor compromisso entre as piores e melhores escolhas da Natureza?

As soluções Lower-First e Upper-First são exemplos de soluções que fazem esse compromisso. Para definirmos essas soluções formalmente temos antes que introduzir a noção de ordenação entre intervalos da função valor.

\subsubsection{Ordem entre intervalos}

Sabemos que soluções ótimas para jogos são baseadas numa definição de políticas de equilíbrio que, por sua vez, depende de uma definição de ordem entre os valores de diferentes pares de políticas (vide Definição 2.7).

Num AMG com probabilidades precisas, dado os conjuntos $\Pi$ e $\Phi$ de políticas do Jogador I e II, respectivamente, a função valor $V(\pi, \phi)(s)$ é definida como $V: \Pi \times \Phi \times S \rightarrow \mathbb{R}$ e portanto a relação de ordem entre os valores de pares de políticas é a mesma definida para o conjunto dos números reais. Já num AMG-IP, a função valor do estado, seguindo um par de políticas $\pi$ e $\phi$, define uma função intervalo $V(\pi, \phi)(s)$ dada por:

$$
\mathcal{V}: \Pi \times \Phi \times S \rightarrow \mathbf{I R}
$$

sendo IR o conjunto de todos os intervalos fechados dos reais, ou seja:

$$
\mathbf{I R}=\{[a, b] \mid a, b \in \mathbb{R}, a \leq b\},
$$

que chamamos de $[\underline{V}(\pi, \phi)(s), \bar{V}(\pi, \phi)(s)] \operatorname{com} \underline{V}(\pi, \phi)(s) \leq \bar{V}(\pi, \phi)(s)$. Assim, é preciso estabelecer uma relação de ordem entre esses intervalos para que seja possível definir políticas de equilíbrio para AMG-IPs.

Chang (2006) utiliza a definição de ordem entre intervalos $\leq_{\text {rest }}$ (ordenação restritiva), enquanto Givan et al. (2000) define as relações de ordem entre intervalos $\leq_{\text {opt }}$ (ordenação otimista) e $\leq_{\text {pes }}$ (ordenação pessimista), conforme mostrado a seguir.

Dados dois intervalos fechados sobre IR, $\left[l_{1}, u_{1}\right]$ e $\left[l_{2}, u_{2}\right]$, podemos definir três relações de ordem diferentes:

Definição 4.2 (Ordenação restritiva entre intervalos). . Dado dois intervalos $\left[l_{1}, u_{1}\right] e\left[l_{2}, u_{2}\right] \in \boldsymbol{I}$, definimos o operador de ordem parcial entre intervalos, $<_{\text {rest }}$ (ordenação restritiva) da seguinte maneira:

$$
\begin{aligned}
& {\left[l_{1}, u_{1}\right] \quad \leq_{\text {rest }}\left[l_{2}, u_{2}\right] \Leftrightarrow\left(l_{1} \leq l_{2}\right) \quad e \quad\left(u_{1} \leq u_{2}\right)} \\
& {\left[l_{1}, u_{1}\right]<_{\text {rest }}\left[l_{2}, u_{2}\right] \Leftrightarrow\left[l_{1}, u_{1}\right] \leq_{\text {rest }}\left[l_{2}, u_{2}\right] \quad e \quad\left[l_{1}, u_{1}\right] \neq\left[l_{2}, u_{2}\right] \text {. }}
\end{aligned}
$$

Definição 4.3 (Ordenação pessimista entre intervalos). . Dado dois intervalos $\left[l_{1}, u_{1}\right] e\left[l_{2}, u_{2}\right] \in \boldsymbol{I} \boldsymbol{R}$, definimos o operador de ordem total entre intervalos, $<_{\text {pes }}$ (ordenação pessimista) da seguinte maneira:

$$
\begin{aligned}
& {\left[l_{1}, u_{1}\right] \leq_{\text {pes }}\left[l_{2}, u_{2}\right] \Leftrightarrow\left\{\begin{array}{lll}
\text { caso } 1: & \left(l_{1}<l_{2}\right) & \text { ou } \\
\text { caso } 2: & \left(l_{1}=l_{2} \quad e \quad u_{1} \leq u_{2}\right)
\end{array}\right.} \\
& {\left[l_{1}, u_{1}\right]<_{\text {pes }}\left[l_{2}, u_{2}\right] \Leftrightarrow\left[l_{1}, u_{1}\right] \leq_{\text {pes }}\left[l_{2}, u_{2}\right] \quad e \quad\left[l_{1}, u_{1}\right] \neq\left[l_{2}, u_{2}\right]}
\end{aligned}
$$

Definição 4.4 (Ordenação otimista entre intervalos). . Dado dois intervalos $\left[l_{1}, u_{1}\right] e\left[l_{2}, u_{2}\right] \in \mathbf{I R}$, definimos o operador de ordem total entre intervalos, $<_{\text {opt }}$ (ordenação otimista) da seguinte maneira:

$$
\begin{aligned}
& {\left[l_{1}, u_{1}\right] \leq_{\text {opt }}\left[l_{2}, u_{2}\right] \Leftrightarrow\left\{\begin{array}{lll}
\text { caso } 1: & \left(u_{1}<u_{2}\right) \quad \text { ou } \\
\text { caso } 2: & \left(u_{1}=u_{2} \quad \text { e } \quad l_{1} \leq l_{2}\right)
\end{array}\right.} \\
& {\left[l_{1}, u_{1}\right]<_{o p t}\left[l_{2}, u_{2}\right] \Leftrightarrow\left[l_{1}, u_{1}\right] \leq_{o p t}\left[l_{2}, u_{2}\right] \quad e \quad\left[l_{1}, u_{1}\right] \neq\left[l_{2}, u_{2}\right]}
\end{aligned}
$$


A Tabela 4.1 aplica as três definições de ordenação, $\leq_{r e s t}, \leq_{p e s} \mathrm{e} \leq_{o p t}$ para dois intervalos dados considerando todas as possíveis relações entre eles, sendo o intervalo marcado com uma elipse, o maior intervalo segundo as definições de ordem (Definição 4.2, 4.3,4.4). Note que no caso de intervalos do tipo "contidos", isto é, em que um intervalo está contido dentro do outro, o operador $\leq_{\text {pes }}$ classifica como maior o intervalo interno, o operador $\leq_{\text {opt }}$ classifica como maior o intervalo externo e o operador $\leq_{\text {rest }}$ não é capaz de determinar qual dos dois intervalos é o maior (isto é, esses intervalos não são ordenáveis). Nos demais casos, as ordens coincidem para os três operadores de ordem.

\begin{tabular}{|c|c|c|c|c|}
\hline & R1 & $\mathrm{R} 2$ & R3 & $\mathrm{R} 4$ \\
\hline$\leq_{\text {rest }}$ & $\stackrel{\longmapsto}{\longmapsto}$ & $\stackrel{1}{\longmapsto}$ & $\stackrel{\longmapsto}{\longmapsto}$ & $\longmapsto \rightleftharpoons$ \\
\hline$\leq_{p e s}$ & $\stackrel{\longmapsto}{\longrightarrow}$ & $\stackrel{1}{\longmapsto}$ & $\stackrel{\longrightarrow}{\longmapsto}$ & $\stackrel{1}{\longmapsto}$ \\
\hline$\leq_{o p t}$ & $\stackrel{\longmapsto}{\longmapsto}$ & $\longmapsto$ & $\stackrel{\longrightarrow}{\longmapsto}$ & $\stackrel{1}{\longmapsto}$ \\
\hline
\end{tabular}

Tabela 4.1: Conjunto completo de relação entre dois intervalos $(R 1, R 2, R 3$ e $R 4)$ e as diferentes definições de ordenação entre eles. Os intervalos destacados são os maiores de acordo com a definição de ordem entre intervalos escolhida.

\subsection{Diferentes critérios para escolha de pares de políticas de equilíbrio de um AMG-IP}

Considerando as relações de ordem entre intervalos, $\leq_{r e s t}, \leq_{p e s} \mathrm{e} \leq_{o p t}$, podemos definir diferentes políticas de equilíbrio, de acordo com o critério de preferência adotado pelos jogadores diante das escolhas da Natureza num jogo AMG-IP, entre eles:

- Lower-Only: critério pessimista e robusto: seleciona o melhor par de políticas de equilíbrio no pior caso (assume a Natureza adversarial). Esse critério não compara intervalos para encontrar políticas de equilíbrio, mas compara somente os limites inferiores da função valor intervalar.

- Upper-Only: critério muito otimista: seleciona o melhor par de políticas de equilíbrio no melhor caso (assume a Natureza cooperativa). Esse critério não compara intervalos para encontrar políticas de equilíbrio, mas compara somente os limites superiores da função valor intervalar.

- Lower-First: esse critério corresponde a uma versão melhorada do critério Lower-Only, pois ainda garante a robustez: "dentre os pares de políticas ótimas de equilíbrio supondo a Natureza adversarial, escolhemos um par de políticas de equilíbrio assumindo agora a Natureza cooperativa". Essa solução compara intervalos para encontrar políticas de equilíbrio segundo a Definição 4.3 de ordenação pessimista entre intervalos $\left(\leq_{\text {pes }}\right)$ (analogamente à solução descrita em Givan et al. (2000) para BMDPs).

- Upper-First: esse critério corresponde a uma versão melhorada do critério Upper-Only: "dentre os pares de políticas ótimas de equilíbrio supondo a Natureza cooperativa, escolhemos um par de políticas de equilíbrio assumindo agora a Natureza adversarial". Essa solução compara intervalos para encontrar políticas de equilíbrio segundo a Definição 4.4 de ordenação otimista entre intervalos $\left(\leq_{\text {opt }}\right)$. Veremos que apesar de Chang (2006) definir políticas de equilíbrio usando a Definição 4.2 de ordenação restritiva entre intervalos $\left(\leq_{\text {rest }}\right)$, a solução que ele propõe escolhe políticas segundo uma ordenação otimista entre intervalos ( $\leq_{o p t}$ ) (Essa solução é análoga à solução descrita em Givan et al. (2000) para BMDPs.

- $\epsilon$-Lower-First e $\epsilon$-Upper-First: esses critérios permitem uma tolerância $\epsilon$ na primeira fase das soluções Lower-First e Upper-First, respectivamente. Mostramos a seguir que a inclusão de um $\epsilon$ nos critérios Lower-First e Upper-First sempre podem melhorar as políticas encontradas por esses critérios, nunca piorar. 
- $\eta$-optimal: esse critério de preferência permite uma maior flexibilidade sobre as suposições de escolhas da Natureza, considerando uma combinação linear entre $\underline{V}^{*}(s)$ e $\bar{V}^{*}(s)$. Dependendo do valor assumido por um parâmetro $\eta$, que pode variar entre 0 e 1 , o jogador pode definir uma medida de equilíbrio entre uma suposição mais otimista ou pessimista sobre o comportamento da Natureza.

Nas próximas seções discutimos em detalhes cada um desses critérios e para cada um definimos políticas de equilíbrio usando as relações de ordem das Definições 4.2, 4.4 e 4.3.

\subsubsection{Critério de preferência Lower-Only}

O critério de preferência Lower-Only considera apenas o limite inferior da função valor intervalar ótima do AMG-IP (Equação (4.4)) e é o critério geralmente adotado para tratar MDPs com probabilidades imprecisas (Seção 2.2) (Satia e Lave Jr, 1973; White III e Eldeib 1994) pois oferece uma abordagem razoável quando procuramos por políticas robustas para um agente (maximizar a recompensa no pior caso de escolhas da Natureza). Ele é um critério robusto porque garante que o Jogador I não perderá mais do que um valor $V^{*}(s)$ para $s \in S$.

A Equação (4.4) calcula $\underline{V}^{*}(s)$ segundo o critério Lower-First, sendo que o par de políticas ótimas $\left\langle\pi^{*}, \phi^{*}\right\rangle$ é calculado por:

$$
\left\langle\pi^{*}, \phi^{*}\right\rangle=\arg \max _{a_{1} \in A_{1}} \min _{a_{2} \in A_{2}}\left(R\left(s, a_{1}, a_{2}\right)+\gamma \min _{p \in K\left(s, a_{1}, a_{2}\right)} \sum_{s^{\prime} \in S} p\left(s^{\prime} \mid s, a_{1}, a_{2}\right) \underline{V}^{*}\left(s^{\prime}\right)\right) .
$$

É fácil provar que $\left\langle\pi^{*}, \phi^{*}\right\rangle$ é um par de políticas de equilíbrio de acordo com a Definição 4.5 dada a seguir, uma vez que ela obedece a definição de equilíbrio de um AMG (Definição 2.7), ao fixarmos as escolhas da Natureza para um $p \in K$ mínimo.

Definição 4.5 (Par de políticas de equilíbrio para AMG-IP - critério pessimista). Um par da políticas $\pi^{*} \in \Pi$ e $\phi^{*} \in \Phi$ é um par de políticas de equilíbrio se não existe uma política $\pi \in \Pi$ tal que

$$
\underline{V}\left(\pi^{*}, \phi^{*}\right)(s)<\underline{V}\left(\pi, \phi^{*}\right)(s), \quad s \in S,
$$

e não existe uma política $\phi \in \Phi$ tal que:

$$
\underline{V}\left(\pi^{*}, \phi\right)(s)<\underline{V}\left(\pi^{*}, \phi^{*}\right)(s), \quad s \in S .
$$

isto é, as políticas $\pi^{*}$ e $\phi^{*}$ são escolhidas de forma que o Jogador I não possa receber $\underline{V}(s)$ maior mudando suas ações e o Jogador II não possa receber um $\underline{V}(s)$ menor mudando suas ações.

O Algoritmo 9 (LOWER-ONLY) recebe como parâmetros de entrada um AMG-IP dado pela tupla $\left\langle S, A_{1}, A_{2}, R, K, \gamma\right\rangle$, o número máximo de iterações e devolve como resposta um par de políticas $\left\langle\pi^{*}, \phi^{*}\right\rangle$ e os conjuntos de todas as ações dos jogadores I e II que alcançam $\underline{V}^{*}(s)$, que chamamos de $A_{1}\left[\underline{V}^{*}\right]$ e $A_{2}\left[\underline{V}^{*}\right]$ (esses conjuntos são usados pelos critérios Lower-First e $\epsilon$-Lower-First nas Seções 4.2.3 e 4.2.6).

A complexidade de resolver um AMG-IP usando o critério Lower-Only é maior do que resolver um AMG (Seção 2.3.2), isso porque o critério Lower-Only faz chamadas a um NLP-Solver. Apesar desse trabalho não focar em algoritmos eficientes, a exemplo das soluções eficientes para MDP-IPs (Seção 2.2.3), para melhorar o Algoritmo 9 é preciso explorar técnicas que minimizem o número de chamadas ao NLPSolver.

\subsubsection{Critério de preferência Upper-Only}

Ao contrário do critério de preferência Lower-Only, que é muito pessimista, podemos definir um critério otimista do ponto de vista do Jogador I. O critério Upper-Only é análogo ao critério Lower-Only mas considera apenas o limite superior da função valor intervalar ótima do AMG-IP (Equação (4.7)) e por isso é um critério otimista. A Equação (4.7) calcula $\bar{V}^{*}(s)$ segundo o critério Upper-First e o par de políticas ótimas $\left\langle\pi^{*}, \phi^{*}\right\rangle$ é calculado por: 


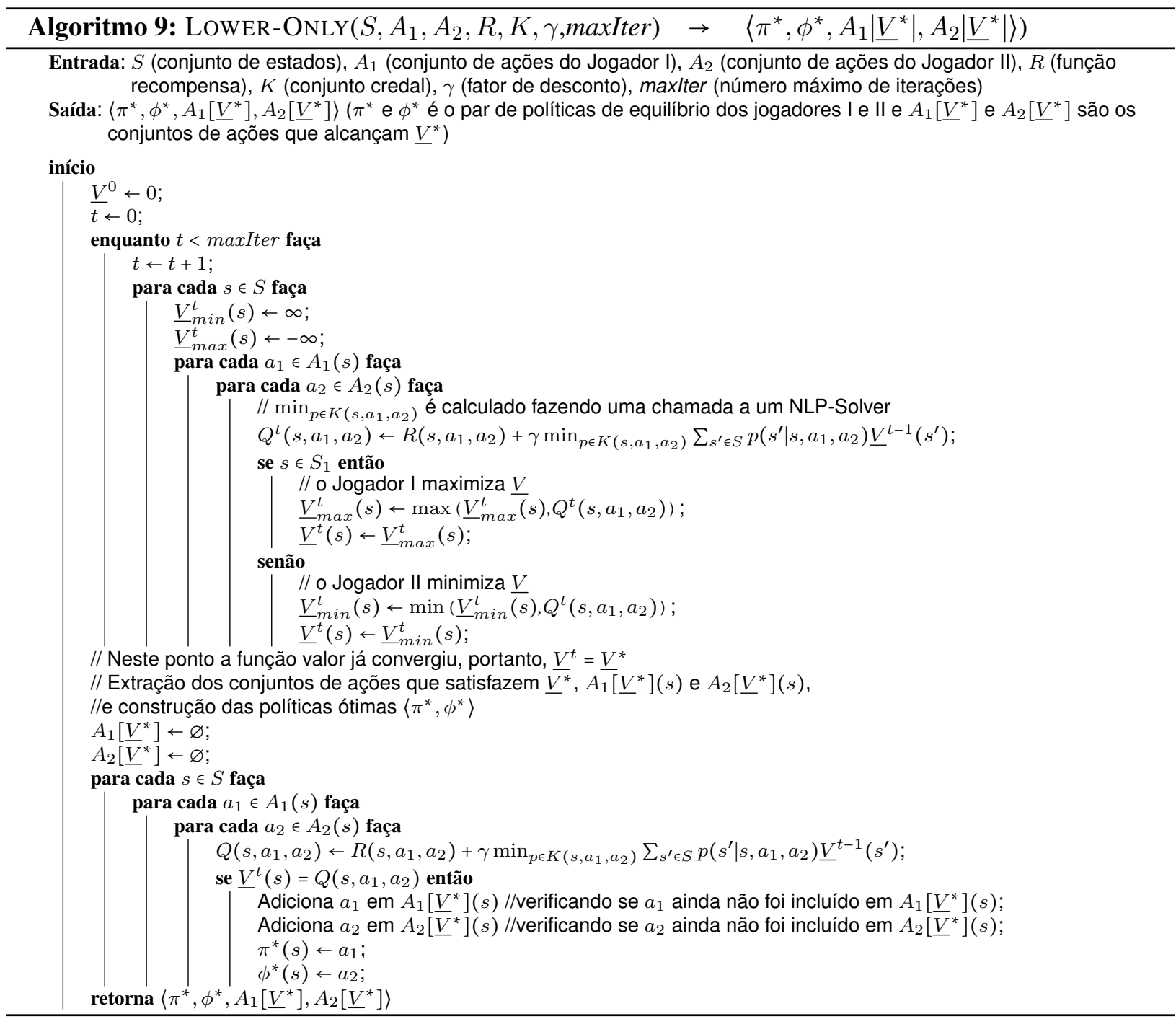

$$
\left\langle\pi^{*}, \phi^{*}\right\rangle=\arg \max _{a_{1} \in A_{1}} \min _{a_{2} \in A_{2}}\left(R\left(s, a_{1}, a_{2}\right)+\gamma \max _{p \in K\left(s, a_{1}, a_{2}\right)} \sum_{s^{\prime} \in S} p\left(s^{\prime} \mid s, a_{1}, a_{2}\right) \bar{V}^{*}\left(s^{\prime}\right)\right) .
$$

É fácil demonstrar que $\left\langle\pi^{*}, \phi^{*}\right\rangle$ é um par de políticas de equilíbrio, de acordo com a Definição 4.6 dada a seguir, uma vez que ela também corresponde à definição de equilíbrio de um AMG (Definição 2.7), ao fixarmos as escolhas da Natureza para um $p \in K$ máximo e assim só considerarmos o limite superior da função valor intervalar.

Definição 4.6 (Par de políticas de equilíbrio para AMG-IP - critério otimista). Um par da políticas $\pi^{*} \in \Pi e \phi^{*} \in \Phi$ é um par de políticas de equilíbrio se não existe uma política $\pi \in \Pi$ tal que

$$
\bar{V}\left(\pi^{*}, \phi^{*}\right)(s)<\bar{V}\left(\pi, \phi^{*}\right)(s), \quad s \in S,
$$

e não existe uma política $\phi \in \Phi$ tal que:

$$
\bar{V}\left(\pi^{*}, \phi\right)(s)<\bar{V}\left(\pi^{*}, \phi^{*}\right)(s), \quad s \in S .
$$

O Algoritmo 10 (UPPER-ONLY) recebe como entrada um AMG-IP dado pela tupla $\left\langle S, A_{1}, A_{2}, R, K, \gamma\right\rangle$, o número de iterações e tem como resultado um par de políticas $\left\langle\pi^{*}, \phi^{*}\right\rangle$ e os conjuntos de todas as ações 


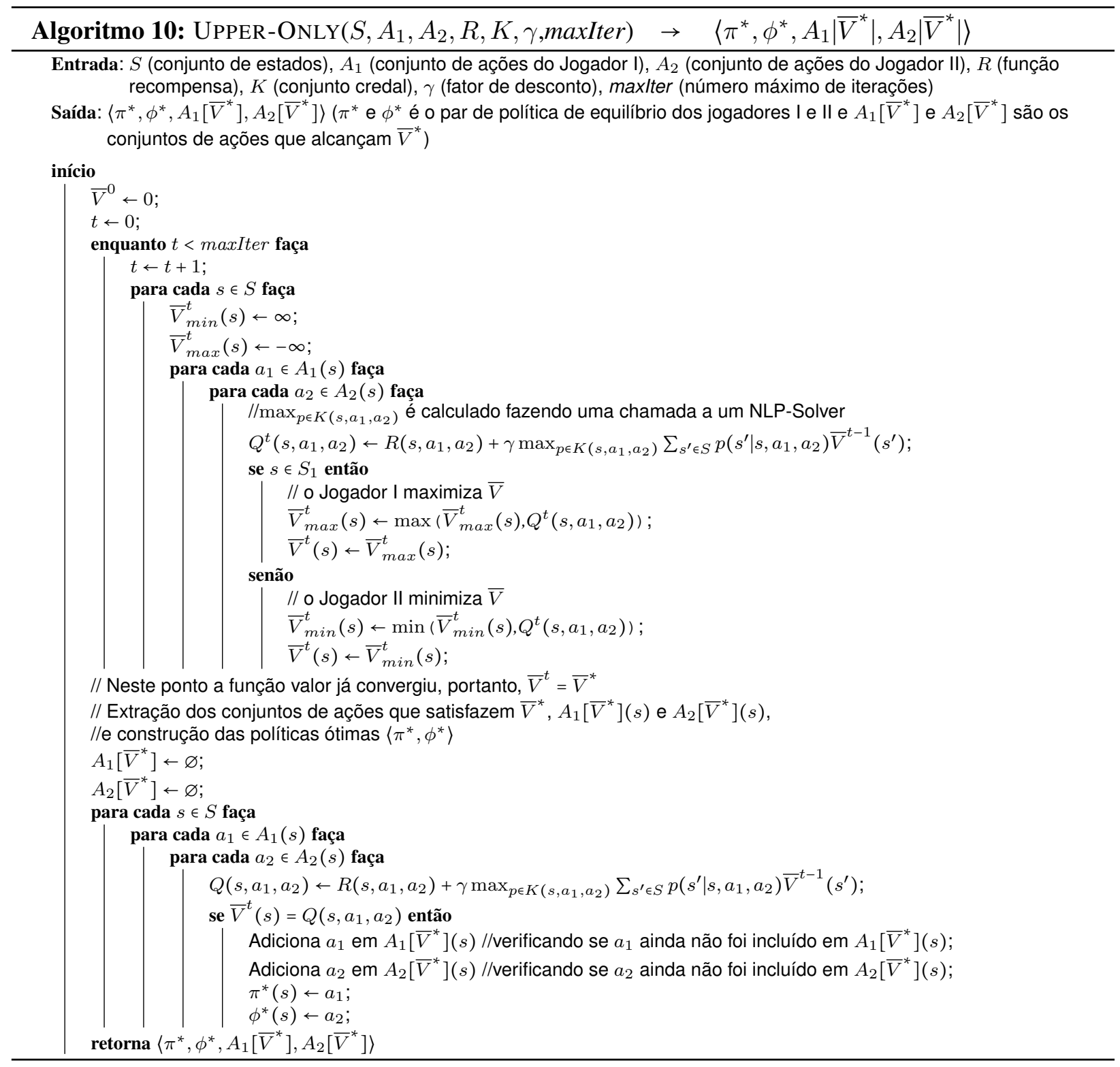

que alcançam $\bar{V}^{*}(s)$. Chamamos de $A_{1}\left[\bar{V}^{*}\right]$ e $A_{2}\left[\bar{V}^{*}\right]$ (que são usados pelos critérios Upper-First e $\epsilon$ Upper-First).

A complexidade de resolver um AMG-IP usando o critério Upper-Only é igual a de se resolver um AMG-IP usando o critério Lower-Only, ou seja, é maior do que resolver um AMG (Seção 2.3.2). Isso também se deve ao fato de que o critério Upper-Only faz chamadas a um NLP-Solver e, assim como no caso do Algoritmo 9, para melhorar o Algoritmo 10 é preciso explorar técnicas que minimizem o número de chamadas ao NLP-Solver.

\subsubsection{Critério de preferência Lower-First}

Enquanto o critério de preferência Lower-Only é pessimista demais, o critério Upper-Only é otimista demais. Nessa seção mostramos uma forma de combinar esses dois critérios, assim como em Givan et al. (2000) para BMDPs, usando o operador $\leq_{\text {pes }}$.

Seja o par de políticas $\langle\pi, \phi\rangle$ encontrado pelo critério Lower-Only, isto é, $\underline{V}(\pi, \phi)(s)=\underline{V}^{*}(s)$. Suponha que exista outro par de políticas ótimas $\left\langle\pi^{\prime}, \phi^{\prime}\right\rangle$ que também satisfaz a igualdade $\underline{V}\left(\pi^{\prime}, \bar{\phi}^{\prime}\right)(s)=\underline{V}^{*}(s)$ (Figura 4.3). Suponha ainda que os limites superiores da função valor calculados para esses dois pares de políticas ótimas sejam diferentes e $\bar{V}\left(\pi^{\prime}, \phi^{\prime}\right)(s)>\bar{V}(\pi, \phi)(s)$ (Figura 4.3). Nesse caso, a escolha do par $\left\langle\pi^{\prime}, \phi^{\prime}\right\rangle$ para o Jogador I (max) é a melhor, uma vez que além de garantir a melhor escolha no pior caso $\underline{V}^{*}$, possibilita ao Jogador I uma recompensa esperada maior no melhor caso de escolha da Natureza $\left(\bar{V}^{\prime}(s)\right)$. 


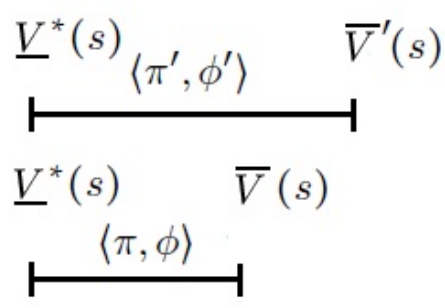

Figura 4.3: Comparação entre possíveis valores da função valor intervalar $\left[\underline{V}^{*}(s), \bar{V}(s)\right] e\left[\underline{V}^{*}(s), \bar{V}^{\prime}(s)\right], \forall s \in S$ geradas pelos pares de políticas ótimas $\langle\pi, \phi\rangle$ e $\left\langle\pi^{\prime}, \phi^{\prime}\right\rangle$ calculadas segundo o critério de preferência Lower-Only.

Para calcular um par de políticas de equilíbrio segundo o critério Lower-First, calculamos primeiro os conjuntos de pares de políticas de equilíbrio que satisfazem $\underline{V}^{*}(s)$ (Equação (4.4)), que chamamos de $A_{1}\left[\underline{V}^{*}\right](s)$ e $A_{2}\left[\underline{V}^{*}\right](s)$, respectivamente. Em seguida, escolhemos um par de políticas ótimas calculando o limite superior da função valor, mas agora consideramos apenas as ações dos conjuntos $A_{1}=A_{1}\left[\underline{V}^{*}\right]$ e $A_{2}=A_{2}\left[\underline{V}^{*}\right]$. Assim, a solução para o critério Lower-First é dada pelos seguintes pares de equações:

$$
\underline{V}^{*}(s)=\max _{a_{1} \in A_{1}} \min _{a_{2} \in A_{2}}\left(R\left(s, a_{1}, a_{2}\right)+\gamma \min _{p \in K\left(s, a_{1}, a_{2}\right)} \sum_{s^{\prime} \in S} p\left(s^{\prime} \mid s, a_{1}, a_{2}\right) \underline{V}^{*}\left(s^{\prime}\right)\right)
$$

e

$$
\bar{V}^{*}(s)=\max _{a_{1} \in A_{1}\left[\underline{V}^{*}\right](s)} \min _{a_{2} \in A_{2}\left[\underline{V}^{*}\right](s)}\left(R\left(s, a_{1}, a_{2}\right)+\gamma \max _{p \in K\left(s, a_{1}, a_{2}\right)} \sum_{s^{\prime} \in S} p\left(s^{\prime} \mid s, a_{1}, a_{2}\right) \bar{V}^{*}\left(s^{\prime}\right)\right),
$$

sendo o $\operatorname{par}\left\langle\pi^{*}, \phi^{*}\right\rangle$ calculado por:

$$
\left\langle\pi^{*}, \phi^{*}\right\rangle=\arg \max _{a_{1} \in A_{1}\left[\underline{V}^{*}\right](s)} \min _{a_{2} \in A_{2}\left[\underline{V}^{*}\right](s)}\left(R\left(s, a_{1}, a_{2}\right)+\gamma \max _{p \in K\left(s, a_{1}, a_{2}\right)} \sum_{s^{\prime} \in S} p\left(s^{\prime} \mid s, a_{1}, a_{2}\right) \bar{V}^{*}\left(s^{\prime}\right)\right) .
$$

Note que na Equação (4.15), $\bar{V}^{*}(s)$ é calculada fazendo $\max$ sobre $a_{1} \in A_{1}\left[\underline{V}^{*}\right]$ e $\min$ sobre $a_{2} \in$ $A_{2}\left[\underline{V}^{*}\right]$, ou seja, considerando todas as ações que satisfazem $\underline{V}^{*}(s)$, calculamos $\bar{V}^{*}(s)$.

O Teorema 4.1 dado a seguir garante que o par de políticas $\left\langle\pi^{*}, \phi^{*}\right\rangle$ encontrados com o critério LowerFirst é um par de políticas de equilíbrio de acordo com a Definição 4.7 de equilíbrio, adotando a Definição 4.3 de ordenação pessimista entre intervalos.

Definição 4.7 (Par de políticas de equilíbrio para AMG-IP - critério pessimista melhorado). Um par da políticas $\pi^{*} \in \Pi e \phi^{*} \in \Phi$ é um par de políticas de equilíbrio segundo a relação de ordenação $\leq_{\text {pes }}$ (Definição 4.3) se não existe uma política $\pi \in \Pi$ tal que

$$
\mathcal{V}\left(\pi^{*}, \phi^{*}\right)(s)<_{\text {pes }} \mathcal{V}\left(\pi, \phi^{*}\right)(s), \quad s \in S,
$$

e não existe uma política $\phi \in \Phi$ tal que:

$$
\mathcal{V}\left(\pi^{*}, \phi\right)(s)<_{\text {pes }} \mathcal{V}\left(\pi^{*}, \phi^{*}\right)(s), \quad s \in S .
$$

sendo $\mathcal{V}\left(\pi^{\prime}, \phi^{\prime}\right)(s) \equiv\left[\underline{V}\left(\pi^{\prime}, \phi^{\prime}\right)(s), \bar{V}\left(\pi^{\prime}, \phi^{\prime}\right)(s)\right]$ para todo $\pi^{\prime} \in \Pi e \phi^{\prime} \in \Phi$.

Teorema 4.1. Um par de políticas $\pi^{*} \in \Pi e \phi^{*} \in \Phi$ encontrados pelo critério Lower-First é um par de políticas de equilíbrio (ótimo) de acordo com a Definição 4.7 . 
Ideia da prova: precisamos demonstrar que:

$$
\begin{aligned}
& (A .1) \quad \nexists \pi \in \Pi \text { tal que } \mathcal{V}\left(\pi^{*}, \phi^{*}\right)(s)<_{\text {pes }} \mathcal{V}\left(\pi, \phi^{*}\right)(s) \quad \mathrm{e} \\
& (A .2) \quad \nexists \phi \in \Phi \text { tal que } \mathcal{V}\left(\pi^{*}, \phi\right)(s)<_{\text {pes }} \mathcal{V}\left(\pi^{*}, \phi^{*}\right)(s) .
\end{aligned}
$$

É importante recordar que $\mathcal{V}(s)$ é a função valor para o Jogador I e, portanto, deve ser maximizada quando a escolha for do Jogador I (escolha entre $\pi$ e $\pi^{*}$ ) e minimizada quando a escolha for do Jogador II (escolha entre $\phi$ e $\phi^{*}$ ).

\section{Demonstração de (A.1).}

Uma vez que o par de políticas ótimas $\left\langle\pi^{*}, \phi^{*}\right\rangle$, devolvido pelo critério Lower-First, satisfaz a Equação (4.14) com relação às escolhas do Jogador I (maximizador), podemos garantir que não existe $\pi \in \Pi$ tal que:

$$
\underline{V}\left(\pi^{*}, \phi^{*}\right)(s)<\underline{V}\left(\pi, \phi^{*}\right)(s),
$$

isto é, $\underline{V}\left(\pi^{*}, \phi^{*}\right)(s) \geq \underline{V}\left(\pi, \phi^{*}\right)(s)$ para $\forall \pi \in \Pi$, ou seja, qualquer outra escolha de política $\pi$ do Jogador I permitiria um ganho menor para o Jogador I. Temos portanto que analisar as duas possibilidades:

$$
\begin{array}{ll}
A .1 .1 & \underline{V}\left(\pi^{*}, \phi^{*}\right)(s)>\underline{V}\left(\pi, \phi^{*}\right)(s) \quad \mathrm{e} \\
A .1 .2 & \underline{V}\left(\pi^{*}, \phi^{*}\right)(s)=\underline{V}\left(\pi, \phi^{*}\right)(s) .
\end{array}
$$

Fazendo a suposição que (A.1.1) é verdade (o que corresponde às relações entre intervalos R1, R3 e R4 da Tabela 4.1), podemos concluir que

$$
\mathcal{V}\left(\pi^{*}, \phi^{*}\right)(s)>_{\text {pes }} \mathcal{V}\left(\pi, \phi^{*}\right)(s),
$$

(caso 1 da Definição 4.3) o que não contradiz (A.1).

Fazendo a suposição que (A.1.2) é verdade (o que corresponde à relação entre intervalos $\mathrm{R} 2$ da Tabela 4.1 de empate nos limites inferiores dos intervalos), tanto $\pi^{*}(s)$ como $\pi(s)$ devem pertencer ao conjunto de ações $A_{1}\left[\underline{V}^{*}\right](s)$ que são usados na segunda fase do critério Lower-First, que garante (Equação (4.15)) e que a escolha ótima do Jogador I (maximizador), $\pi^{*}(s)$, garante que:

$$
\bar{V}\left(\pi^{*}, \phi^{*}\right)(s) \geq \bar{V}\left(\pi, \phi^{*}\right)(s) .
$$

Assim, dado que as condições (A.1.2) e (4.20) correspondem ao caso 2 da Definição 4.3, temos:

$$
\mathcal{V}\left(\pi^{*}, \phi^{*}\right)(s)>_{\text {pes }} \mathcal{V}\left(\pi, \phi^{*}\right)(s),
$$

que também não contradiz (A.1). Portanto como $(A .1 .1)$ e (A.1.2) são todos os casos possíveis para os pares de políticas $\left\langle\pi^{*}, \phi^{*}\right\rangle$ e $\left\langle\pi, \phi^{*}\right\rangle$, podemos concluir que:

$$
\nexists \pi \in \Pi \quad \text { tal que } \quad \mathcal{V}\left(\pi^{*}, \phi^{*}\right)(s)<_{\text {pes }} \mathcal{V}\left(\pi, \phi^{*}\right)(s) \text {, }
$$

como queríamos demonstrar. 


\section{Demonstração de (A.2).}

Uma vez que o par de políticas ótimas $\left\langle\pi^{*}, \phi^{*}\right\rangle$, devolvido pelo critério Lower-First, satisfaz a Equação (4.14) com relação às escolhas do Jogador II (minimizador), podemos garantir que não existe $\phi \in \Phi$ tal que:

$$
\underline{V}\left(\pi^{*}, \phi\right)(s)<\underline{V}\left(\pi^{*}, \phi^{*}\right)(s),
$$

isto é, $\underline{V}\left(\pi^{*}, \phi\right)(s) \geq \underline{V}\left(\pi^{*}, \phi^{*}\right)(s)$ para $\forall \phi \in \Phi$, ou seja, qualquer outra escolha de política $\phi$ do Jogador II permitiria um ganho ainda maior para o Jogador I. Temos portanto que analisar as duas possibilidades:

$$
\begin{array}{ll}
A .2 .1 & \underline{V}\left(\pi^{*}, \phi\right)(s)>\underline{V}\left(\pi^{*}, \phi^{*}\right)(s) \quad \text { e } \\
A .2 .2 & \underline{V}\left(\pi^{*}, \phi\right)(s)=\underline{V}\left(\pi^{*}, \phi^{*}\right)(s) .
\end{array}
$$

Fazendo a suposição que (A.2.1) é verdade (o que corresponde às relações entre intervalos R1, R3 e R4 da Tabela 4.1), podemos concluir que

$$
\mathcal{V}\left(\pi^{*}, \phi\right)(s)>_{\text {pes }} \mathcal{V}\left(\pi^{*}, \phi^{*}\right)(s),
$$

(caso 1 da Definição 4.3) o que não contradiz (A.2).

Fazendo a suposição que (A.2.2) é verdade (o que corresponde à relação entre intervalos $\mathrm{R} 2$ da Tabela 4.1 de empate nos limites inferiores dos intervalos), tanto $\phi^{*}(s)$ como $\phi(s)$ devem pertencer ao conjunto de ações $A_{2}\left[\underline{V}^{*}\right](s)$ que são usados na segunda fase do critério Lower-First, que garante (Equação (4.15)) e que a escolha do Jogador II (minimizador), $\phi^{*}(s)$, garante que:

$$
\bar{V}\left(\pi^{*}, \phi\right)(s) \geq \bar{V}\left(\pi^{*}, \phi^{*}\right)(s) .
$$

Assim, dado que as condições (A.2.2) e (4.23) correspondem ao caso 2 da Definição 4.3, temos:

$$
\mathcal{V}\left(\pi^{*}, \phi\right)(s)>_{\text {pes }} \mathcal{V}\left(\pi^{*}, \phi^{*}\right)(s),
$$

que também não contradiz (A.2). Portanto como $(A .2 .1)$ e (A.2.2) são todos os casos possíveis para os pares de políticas $\left\langle\pi^{*}, \phi\right\rangle$ e $\left\langle\pi^{*}, \phi^{*}\right\rangle$, podemos concluir que:

$$
\nexists \phi \in \Phi \text { tal que } \mathcal{V}\left(\pi^{*}, \phi\right)(s)<_{\text {pes }} \mathcal{V}\left(\pi^{*}, \phi^{*}\right)(s) \text {, }
$$

como queríamos demonstrar.

O Algoritmo 11 (LOWER-FIRST) recebe como entrada o AMG-IP dado pela tupla $\left\langle S, A_{1}, A_{2}, R, K, \gamma\right\rangle$ e o número máximo de iterações (maxIter). O algoritmo faz uma chamada ao Algoritmo 9 (LowER-ONLY) para obter os conjuntos de pares de políticas que alcançam $\underline{V}^{*}(s), A_{1}\left[\underline{V}^{*}\right](s)$ e $A_{2}\left[\underline{V}^{*}\right](s)$, para depois fazer uma chamada ao Algoritmo 10 (UPPER-ONLY) passando como parâmetro de entrada $A_{1}\left[V^{*}\right](s)$ e $A_{2}\left[\underline{V}^{*}\right](s)$. O algoritmo devolve o par de políticas de equilíbrio segundo o Teorema 4.1.

O tempo gasto para resolver um AMG-IP usando o critério Lower-First é, no pior dos casos, duas vezes o tempo gasto para resolver o mesmo AMG-IP usando o critério Lower-Only. Isso ocorre porque, no pior dos casos, todas as ações usadas na primeira fase de execução do critério Lower-First alcançam os mesmos valores da função valor intervalar no limite inferior, o que faz com que esses mesmas ações sejam usadas na segunda fase de execução. Sendo assim, a classe de complexidade de resolver um AMG-IP usando o critério Lower-First é a mesma do critério Lower-Only. 


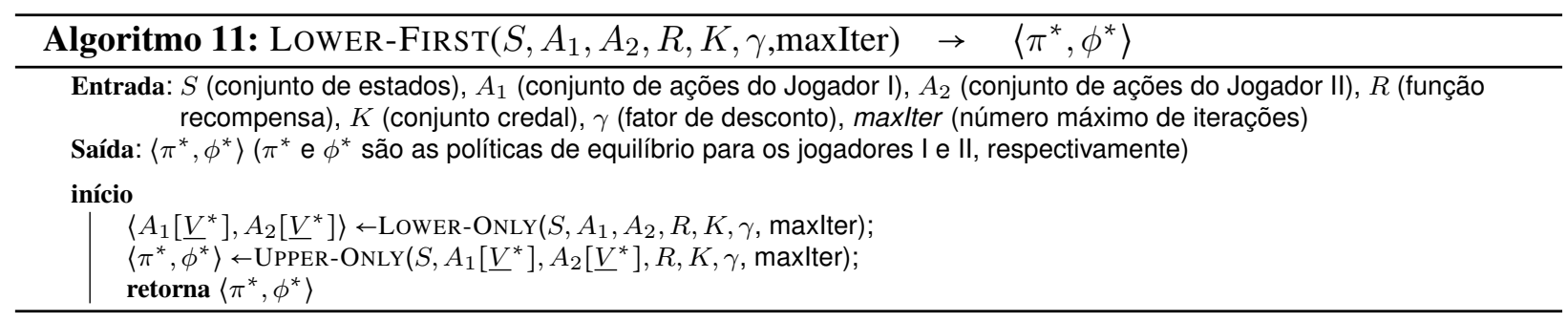

\subsubsection{Critério de preferência Upper-First}

O critério de preferência Upper-First para AMGs com probabilidades imprecisas foi proposto por Chang (2006) usando o operador $\leq_{\text {rest }}$, enquanto que uma versão análoga para BMDPs foi proposta por Givan et al. (2000) usando o operador $\leq_{o p t}$. A diferença entre o critério Lower-First que definimos na Seção 4.2.3 e o critério Upper-First é a ordem em que as Equações (4.14) e (4.15) são executadas. Primeiro, calculamos o valor de $\bar{V}^{*}(s)$ e os conjuntos $A_{1}\left[\bar{V}^{*}\right](s)$ e $A_{2}\left[\bar{V}^{*}\right](s)$ que alcançam $\bar{V}^{*}(s)$ para todo $s \in S$; em seguida, escolhemos um par de políticas ótimas calculando o limite inferior da função valor, $V^{*}(s)$, mas agora considerando apenas as ações em $A_{1}\left[\bar{V}^{*}\right]$ e $A_{2}\left[\bar{V}^{*}\right]$. As equações abaixo calculam o par de políticas ótimas segundo o critério Upper-First:

$$
\bar{V}^{*}(s)=\max _{a_{1} \in A_{1}} \min _{a_{2} \in A_{2}}\left(R\left(s, a_{1}, a_{2}\right)+\gamma \max _{p \in K\left(s, a_{1}, a_{2}\right)} \sum_{s^{\prime} \in S} p\left(s^{\prime} \mid s, a_{1}, a_{2}\right) \bar{V}^{*}\left(s^{\prime}\right)\right)
$$

$\mathrm{e}$

$$
\underline{V}^{*}(s)=\max _{a_{1} \in A_{1}\left[\bar{V}^{*}\right](s)} \min _{a_{2} \in A_{2}\left[\bar{V}^{*}\right](s)}\left(R\left(s, a_{1}, a_{2}\right)+\gamma \min _{p \in K\left(s, a_{1}, a_{2}\right)} \sum_{s^{\prime} \in S} p\left(s^{\prime} \mid s, a_{1}, a_{2}\right) \underline{V}^{*}\left(s^{\prime}\right)\right) .
$$

Note que $\underline{V}^{*}(s)$ é calculado fazendo max sobre $a_{1} \in A_{1}\left[\bar{V}^{*}\right]$ e $\min$ sobre $a_{2} \in A_{2}\left[\bar{V}^{*}\right]$.

Definição 4.8 (Par de políticas de equilíbrio para AMG-IP - critério otimista melhorado). Um par da políticas $\pi^{*} \in \Pi e \phi^{*} \in \Phi$ é um par de políticas de equilíbrio segundo a relação de ordem $\leq_{\text {opt }}$ (Definição 4.4) se não existe uma política $\pi \in \Pi$ tal que

$$
\mathcal{V}\left(\pi^{*}, \phi^{*}\right)(s)<_{o p t} \mathcal{V}\left(\pi, \phi^{*}\right)(s), \quad s \in S,
$$

e não existe uma política $\phi \in \Phi$ tal que:

$$
\mathcal{V}\left(\pi^{*}, \phi\right)(s)<_{\text {opt }} \mathcal{V}\left(\pi^{*}, \phi^{*}\right)(s), \quad s \in S .
$$

Teorema 4.2. $O$ par de políticas $\left\langle\pi^{*}, \phi^{*}\right\rangle$ devolvidos pelo algoritmo Upper-First são políticas de equilíbrio de acordo com a relação de ordem entre intervalos $\leq_{\text {opt }}$.

Ideia da prova: precisamos demonstrar que:

$$
\begin{aligned}
& (B .1) \quad \nexists \pi \in \Pi \text { tal que } \mathcal{V}\left(\pi^{*}, \phi^{*}\right)(s)<_{\text {opt }} \mathcal{V}\left(\pi, \phi^{*}\right)(s) \quad \text { e } \\
& (B .2) \quad \nexists \phi \in \Phi \text { tal que } \mathcal{V}\left(\pi^{*}, \phi\right)(s)<_{\text {opt }} \mathcal{V}\left(\pi^{*}, \phi^{*}\right)(s) .
\end{aligned}
$$

Novamente, é importante recordar que $\mathcal{V}(s)$ é a função valor para o Jogador I e, portanto, deve ser maximizada quando a escolha for do Jogador I (escolha entre $\pi$ e $\pi^{*}$ ) e minimizada quando a escolha for do Jogador II (escolha entre $\phi$ e $\phi^{*}$ ). 
Demonstração de (B.1).

Uma vez que o par de políticas ótimas $\left\langle\pi^{*}, \phi^{*}\right\rangle$, devolvido pelo critério Upper-First, satisfaz a Equação (4.25) com relação às escolhas do Jogador I (maximizador), podemos garantir que não existe $\pi \in \Pi$ tal que:

$$
\bar{V}\left(\pi^{*}, \phi^{*}\right)(s)<\bar{V}\left(\pi, \phi^{*}\right)(s),
$$

isto é, $\bar{V}\left(\pi^{*}, \phi^{*}\right)(s) \geq \bar{V}\left(\pi, \phi^{*}\right)(s)$ para $\forall \pi \in \Pi$, ou seja, qualquer outra escolha de política $\pi$ do Jogador I permitiria um ganho menor para o Jogador I. Temos portanto que analisar as duas possibilidades:

$$
\begin{array}{ll}
\text { B.1.1 } & \bar{V}\left(\pi^{*}, \phi^{*}\right)(s)>\bar{V}\left(\pi, \phi^{*}\right)(s) \quad \text { e } \\
\text { B.1.2 } & \bar{V}\left(\pi^{*}, \phi^{*}\right)(s)=\bar{V}\left(\pi, \phi^{*}\right)(s) .
\end{array}
$$

Fazendo a suposição que (B.1.1) é verdade (o que corresponde às relações entre intervalos R1, R2 e R4 da Tabela 4.1), podemos concluir que

$$
\mathcal{V}\left(\pi^{*}, \phi^{*}\right)(s)>_{\text {opt }} \mathcal{V}\left(\pi, \phi^{*}\right)(s),
$$

(caso 1 da Definição 4.4) o que não contradiz (B.1).

Fazendo a suposição que (B.1.2) é verdade (o que corresponde à relação entre intervalos R3 da Tabela 4.1 de empate nos limites superiores dos intervalos), tanto $\pi^{*}(s)$ como $\pi(s)$ devem pertencer ao conjunto de ações $A_{1}\left[\bar{V}^{*}\right](s)$ que são usados na segunda fase do critério Upper-First, que garante (Equação (4.26)) e que a escolha ótima do Jogador I (maximizador), $\pi^{\star}(s)$, garante que:

$$
\underline{V}\left(\pi^{*}, \phi^{*}\right)(s) \geq \underline{V}\left(\pi, \phi^{*}\right)(s) .
$$

Assim, dado que as condições (B.1.2) e (4.30) correspondem ao caso 2 da Definição 4.4, temos:

$$
\mathcal{V}\left(\pi^{*}, \phi^{*}\right)(s)>_{\text {opt }} \mathcal{V}\left(\pi, \phi^{*}\right)(s)
$$

que também não contradiz (B.1). Portanto como (B.1.1) e (B.1.2) são todos os casos possíveis para os pares de políticas $\left\langle\pi^{*}, \phi^{*}\right\rangle$ e $\left\langle\pi, \phi^{*}\right\rangle$, podemos concluir que:

$$
\nexists \pi \in \Pi \quad \text { tal que } \mathcal{V}\left(\pi^{*}, \phi^{*}\right)(s)<_{o p t} \mathcal{V}\left(\pi, \phi^{*}\right)(s)
$$

como queríamos demonstrar.

Demonstração de (B.2).

Uma vez que o par de políticas ótimas $\left\langle\pi^{*}, \phi^{*}\right\rangle$, devolvido pelo critério Upper-First, satisfaz a Equação (4.25) com relação às escolhas do Jogador II (minimizador), podemos garantir que não existe $\phi \in \Phi$ tal que:

$$
\bar{V}\left(\pi^{*}, \phi\right)(s)<\bar{V}\left(\pi^{*}, \phi^{*}\right)(s),
$$

isto é, $\bar{V}\left(\pi^{*}, \phi\right)(s) \geq \bar{V}\left(\pi^{*}, \phi^{*}\right)(s)$ para $\forall \phi \in \Phi$, ou seja, qualquer outra escolha de política $\phi$ do Jogador II permitiria um ganho ainda maior para o Jogador I. Temos portanto que analisar as duas possibilidades:

$$
\begin{array}{ll}
B .2 .1 & \bar{V}\left(\pi^{*}, \phi\right)(s)>\bar{V}\left(\pi^{*}, \phi^{*}\right)(s) \quad \mathrm{e} \\
B .2 .2 & \bar{V}\left(\pi^{*}, \phi\right)(s)=\bar{V}\left(\pi^{*}, \phi^{*}\right)(s) .
\end{array}
$$

Fazendo a suposição que (B.2.1) é verdade (o que corresponde às relações entre intervalos R1, R2 e R4 
da Tabela 4.1), podemos concluir que

$$
\mathcal{V}\left(\pi^{*}, \phi\right)(s)>_{\text {opt }} \mathcal{V}\left(\pi^{*}, \phi^{*}\right)(s)
$$

(caso 1 da Definição 4.4) o que não contradiz (B.2).

Fazendo a suposição que (B.2.2) é verdade (o que corresponde à relação entre intervalos R3 da Tabela 4.1 de empate nos limites superiores dos intervalos), tanto $\phi^{*}(s)$ como $\phi(s)$ devem pertencer ao conjunto de ações $A_{2}\left[\bar{V}^{*}\right](s)$ que são usados na segunda fase do critério Upper-First, que garante (Equação (4.26)) e que a escolha ótima do Jogador II (minimizador), $\phi^{*}(s)$, garante que:

$$
\underline{V}\left(\pi^{*}, \phi\right)(s) \geq \underline{V}\left(\pi^{*}, \phi^{*}\right)(s) .
$$

Assim, dado que as condições (B.2.2) e (4.33) correspondem ao caso 2 da Definição 4.4, temos:

$$
\mathcal{V}\left(\pi^{*}, \phi\right)(s)>_{\text {opt }} \mathcal{V}\left(\pi^{*}, \phi^{*}\right)(s),
$$

que também não contradiz (B.2). Portanto como (B.2.1) e (B.2.2) são todos os casos possíveis para os pares de políticas $\left\langle\pi^{*}, \phi\right\rangle$ e $\left\langle\pi^{*}, \phi^{*}\right\rangle$, podemos concluir que:

$$
\nexists \phi \in \Phi \quad \text { tal que } \quad \mathcal{V}\left(\pi^{*}, \phi\right)(s)<_{o p t} \mathcal{V}\left(\pi^{*}, \phi^{*}\right)(s)
$$

como queríamos demonstrar.

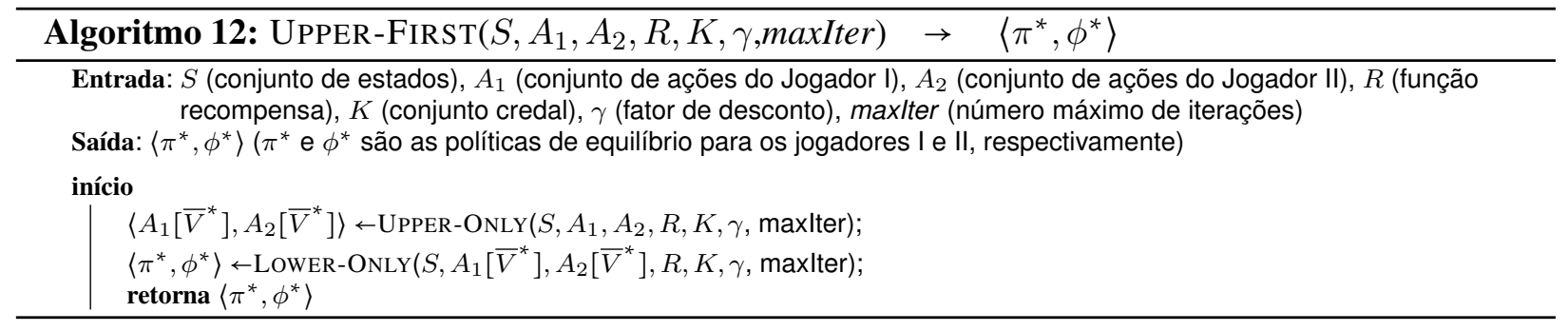

O Algoritmo 12 (UPPER-FIRST) recebe como entrada o AMG-IP dado pela tupla $\left\langle S, A_{1}, A_{2}, R, K, \gamma\right\rangle$ e o número máximo de iterações. Ele primeiro faz uma chamada ao Algoritmo 10 (UPPER-ONLY) para obter os conjuntos de pares de políticas que alcançam $\bar{V}^{*}(s)\left(A_{1}\left[\bar{V}^{*}\right](s)\right.$ e $\left.A_{2}\left[\bar{V}^{*}\right](s)\right)$ para depois fazer uma chamada ao Algoritmo 9 (LOWER-ONLY) passando como parâmetro de entrada $A_{1}\left[\bar{V}^{*}\right](s)$ e $A_{2}\left[\bar{V}^{*}\right](s)$. Chang (2006) provou que esse algoritmo tem como resposta os pares de políticas de equilíbrio segundo a definição de equilíbrio dada a seguir.

Apesar de Chang (2006) ter proposto o algoritmo Upper-First para AMG-INTERVALs ele usa uma definição de políticas de equilíbrio, dada a seguir.

Definição 4.9 (Par de políticas de equilíbrio para AMG-IP (Chang 2006)). Um par da políticas $\pi^{*} \in \Pi$ $e \phi^{*} \in \Phi$ é um par de políticas de equilíbrio segundo a relação de ordenação $\leq_{\text {rest }}$ (Definição 4.2) se não existe uma política $\pi \in \Pi$ tal que

$$
\mathcal{V}\left(\pi^{*}, \phi^{*}\right)(s)<_{\text {rest }} \mathcal{V}\left(\pi, \phi^{*}\right)(s), \quad s \in S,
$$

e não existe uma política $\phi \in \Phi$ tal que:

$$
\mathcal{V}\left(\pi^{*}, \phi\right)(s)<_{\text {rest }} \mathcal{V}\left(\pi^{*}, \phi^{*}\right)(s), \quad s \in S .
$$


Como foi observado em Chang (2006) (Seção 2, página 338), uma vez que essa definição de equilíbrio faz uso da Definição 4.2 (ordenação restritiva entre intervalos), podem existir diferentes pares de políticas de equilíbrio com diferentes funções valor intervalar. De fato, o operador $\leq_{\text {rest }}$ não define uma ordem entre intervalos contidos (Figura 4.4), uma vez que o operador $\leq_{\text {rest }}$ é de ordem parcial.

Apesar de Chang (2006) usar uma definição de equilíbrio baseada em uma ordenação parcial entre intervalos (Definição 4.9), o algoritmo proposto por ele para encontrar pares de políticas de equilíbrio, o algoritmo Upper-First, usa uma ordenação total dos intervalos, ao invés de uma ordenação parcial. Isso é garantido pelo Teorema 4.2.

A principal mudança no trabalho de Chang (2006) ao considerarmos a Definição 4.4 de ordenação de intervalos usada na Definição 4.8 de equilíbrio para o critério Upper-First é poder garantir a escolha entre intervalos contidos: o critério Upper-First sempre escolhe o intervalo externo (coluna R4 da Tabela 4.1).

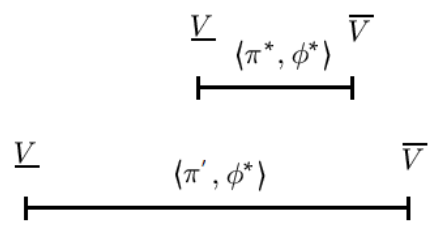

Figura 4.4: Comparação entre possíveis intervalos do tipo contidos da função valor. Usando a Definição 4.9 de pares de políticas de equilíbrio de Chang (2006), ambos os pares, $\left\langle\pi^{*}, \phi^{*}\right\rangle$ e $\left\langle\pi^{*}, \phi^{*}\right\rangle$, são pares de políticas de equilíbrio, enquanto que usando a Definição 4.8, somente o par $\left\langle\pi^{\prime}, \phi^{*}\right\rangle$ é considerado um par de políticas de equilíbrio.

A Figura 4.4 ilustra dois intervalos da função valor intervalar gerados pelos pares de políticas $\left\langle\pi^{*}, \phi^{*}\right\rangle$ e $\left\langle\pi^{\prime}, \phi^{*}\right\rangle$. Note que, de acordo com a Definição 4.9 (que usa a definição de ordem restritiva) os dois pares de políticas são de equilíbrio e de acordo com a Definição 4.8 (que usa a definição de ordem otimista) somente o par $\left\langle\pi^{\prime}, \phi^{*}\right\rangle$ é considerado um par de políticas de equilíbrio.

\subsubsection{Comparação entre os critérios Lower-First e Upper-First}

Chang (2006) propôs o critério de preferência Upper-First para AMGs com probabilidades imprecisas dadas por intervalos que foi estendido na Seção 4.2.4 para AMG-IPs e com uma nova definição de políticas de equilíbrio. Com base no trabalho de Chang (2006), neste trabalho, propomos o critério Lower-First que, a princípio, é uma melhoria do critério robusto Lower-Only para AMG-IPs. A questão que levantamos é: $o$ critério Lower-First pode encontrar políticas que resultam numa recompensa esperada acumulada maior quando comparado ao critério Upper-First?

A resposta para essa pergunta é que depende da configuração dos intervalos da função valor intervalar para os estados do problema.

A Figura 4.5(a) ilustra uma situação em que, diante de um estado $s \in S_{1}$ do Jogador I e usando o critério Lower-First, o par de políticas de equilíbrio escolhido pelo Jogador I é $\left\langle\pi^{*}, \phi^{*}\right\rangle$, pois usando a Definição 4.3 de ordenação pessimista $\mathcal{V}\left(\pi^{*}, \phi^{*}\right)(s)>\mathcal{V}\left(\pi^{\prime}, \phi^{*}\right)(s)$. Por outro lado, usando o critério Upper-First neste mesmo estado $s \in S_{1}$, o par de políticas de equilíbrio escolhido é $\left\langle\pi^{\prime}, \phi^{*}\right\rangle$, pois de acordo com a Definição 4.4 de ordenação otimista $\mathcal{V}\left(\pi^{\prime}, \phi^{*}\right)(s)>\mathcal{V}\left(\pi^{*}, \phi^{*}\right)(s)$. Similarmente, a Figura 4.5(b) ilustra uma situação em que, num estado $s \in S_{2}$ do Jogador II e usando o critério Lower-First, o par de políticas de equilíbrio escolhido pelo Jogador II é $\left\langle\pi^{*}, \phi^{\prime}\right\rangle$ e usando o critério Upper-First neste mesmo estado o par de políticas de equilíbrio escolhido é $\left\langle\pi^{*}, \phi^{*}\right\rangle$.

\subsubsection{Critérios de preferência $\epsilon$-Lower-First e $\epsilon$-Upper-First}

A fase 2 do algoritmo Lower-First só ocorre quando houver "empates" entre as ações que alcançaram um valor mínimo na fase 1 do algoritmo. Da mesma forma, a fase 2 do critério Upper-First (Seção 4.2.4) só é justificada quando há "empates" entre as ações que alcançarem um valor máximo na fase 1 do algoritmo.

Podemos melhorar as escolhas das políticas ótimas com um algoritmo que admita um pequeno intervalo de diferença entre os valores alcançados por cada ação nos limites inferiores ou superiores da função valor intervalar. Chamamos esses critérios de $\epsilon$-Lower-First e $\epsilon$-Upper-First. Assim, as ações escolhidas para a fase 2 de cada algoritmo são consideradas "empatadas", caso a diferença entre os valores alcançados por 


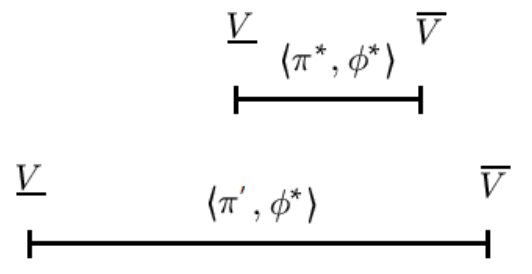

(a) Estados em que o Jogador I faz a escolha de ações
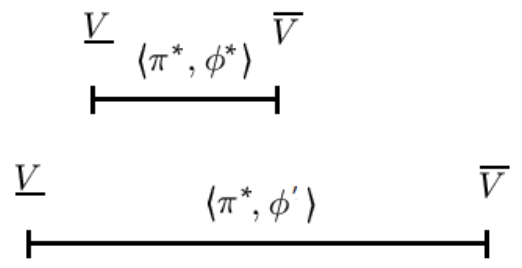

(b) Estados em que o Jogador II faz a escolha de ações

Figura 4.5: Diferentes possibilidades de configuração da função valor intervalar para os critérios Lower-First (LF) e Upper-First $(U F)$.

cada uma delas for menor ou igual a um $\epsilon$.

O Exemplo 4.1 ajuda a entender como a utilização de um $\epsilon$ pode melhorar o critério Lower-First.

Exemplo 4.1. Suponha que estamos usando o critério Lower-First para encontrar um par de políticas de equilíbrio e que na primeira fase do algoritmo Lower-First, em um estágio te para um determinado estado $s \in S_{1}$, temos os seguintes valores para a função $\underline{Q}^{t}$.

$$
\begin{aligned}
\underline{Q}^{t}\left(s, a_{1}, a_{2}\right) & =20.87 \\
\underline{Q}^{t}\left(s, a_{1}^{\prime}, a_{2}\right) & =18.54 \\
\underline{Q} & \left(s, a_{1}^{\prime \prime}, a_{2}\right)=10 \\
\underline{Q}^{t}\left(s, a_{1}^{\prime \prime \prime}, a_{2}\right) & =20.83
\end{aligned}
$$

Assim, a escolha maxmin dos jogadores para a função valor é

$$
\underline{V^{t}}(s)=\max _{a_{1} \in A_{1}} \min _{a_{2} \in A_{2}} \underline{Q}\left(s, a_{1}, a_{2}\right)=20.87,
$$

e o par de ações $\left\langle a_{1}, a_{2}\right\rangle$ é escolhido. Se considerarmos que té o último estágio, ou seja, que em $t$ os valores da função valor já convergiram, temos que $\underline{V}^{t}(s)=\underline{V}^{*}(s)$ e assim $A_{1}\left[\underline{V}^{*}\right](s)=\left\{a_{1}\right\} e$ $A_{2}\left[\underline{V}^{*}\right](s)=\left\{a_{2}\right\}$, o que deixaria a segunda fase do algoritmo Lower-First trivial, pois só há uma escolha de par de ações em s. Porém, ao calcularmos o limite superior $\bar{Q}^{t}$ para essas mesma ações, temos:

$$
\begin{aligned}
\bar{Q}^{t}\left(s, a_{1}, a_{2}^{1}\right) & =40.7 \\
\bar{Q}^{t}\left(s, a_{1}^{\prime}, a_{2}\right) & =38.53 \\
\bar{Q}^{t}\left(s, a_{1}^{\prime \prime}, a_{2}\right) & =16 \\
\bar{Q}^{t}\left(s, a_{1}^{\prime \prime \prime}, a_{2}\right) & =60.15
\end{aligned}
$$

o que indica que o par de ações $\left\langle a_{1}^{\prime \prime \prime}, a_{2}\right\rangle$ dariam um ganho muito maior que o par $\left\langle a_{1}, a_{2}\right\rangle$ com relação ao limite superior.

Como o único par de ações disponível na segunda fase do algoritmo Lower-First é o par $\left\langle a_{1}, a_{2}\right\rangle$, temos que a função valor alcançada pelo algoritmo Lower-First é $\mathcal{V}(s)=[20.87,40.7]$, considerando que $\bar{Q}^{t}=\bar{Q}^{*}$.

Porém, se considerarmos um $\epsilon=0.05$ na primeira fase do algoritmo Lower-First teríamos que $A_{1}\left[\underline{V}^{*}\right](s)$ $=\left\{a_{1}, a_{1}^{\prime \prime \prime}\right\}$ e $A_{2}\left[\underline{V}^{*}\right](s)=\left\{a_{2}\right\}$ e na segunda fase do algoritmo Lower-First a função valor calculada é $\mathcal{V}(s)=[20.5,60.15]$. Ou seja, considerando um pequeno risco $\epsilon$ na primeira fase do algoritmo Lower-First podemos permitir um ganho ainda maior para o Jogador I com relação ao limite superior de $\mathcal{V}^{\star}(s)$. 
Teorema 4.3. $O$ critério $\epsilon$-Lower-First sempre encontra pares de políticas com valor maior ou igual que $o$ critério Lower-First (a menos de um $\epsilon$ com relação ao limite inferior).

\section{Idéia da Prova:}

Seja o par de políticas $\left\langle\pi_{1}, \phi_{1}\right\rangle$ encontrado pelo critério Lower-First. Se houver outro par de políticas $\left\langle\pi_{2}, \phi_{1}\right\rangle$ tal que $\underline{V}\left(\pi_{1}, \phi_{1}\right)(s)-\underline{V}\left(\pi_{2}, \phi_{1}\right)(s) \leq \epsilon \mathrm{e} \bar{V}\left(\pi_{1}, \phi_{1}\right)(s) \leq \bar{V}\left(\pi_{2}, \phi_{1}\right)(s)$, então o critério $\epsilon$-LowerFirst escolherá o par de políticas $\left\langle\pi_{2}, \phi_{1}\right\rangle$. Do contrário, ambos os critérios Lower-First e $\epsilon$-Lower-First escolherão o par de políticas $\left\langle\pi_{1}, \phi_{1}\right\rangle$. Ou seja, o critério $\epsilon$-Lower-First sempre poderá melhorar as escolhas feitas pelo critério Lower-First com relação as escolhas feitas pelo Jogador I.

Demonstração.

Seja o par de políticas $\left\langle\pi_{1}, \phi_{1}\right\rangle$ encontrado pelo critério Lower-First. Suponha que exista outro par de políticas $\left\langle\pi_{2}, \phi_{1}\right\rangle$. Se $\underline{V}\left(\pi_{1}, \phi_{1}\right)(s)-\underline{V}\left(\pi_{2}, \phi_{1}\right)(s)>\epsilon$ então tanto o critério Lower-First quanto o critério $\epsilon$-Lower-First usarão as mesmas ações na primeira e na segunda fase dos algoritmos, e assim a escolha do critério $\epsilon$-Lower-First será a mesma do critério Lower-First, ou seja, escolherá o par $\left\langle\pi_{1}, \phi_{1}\right\rangle$. Esses caso é ilustrado na Figura 4.6.

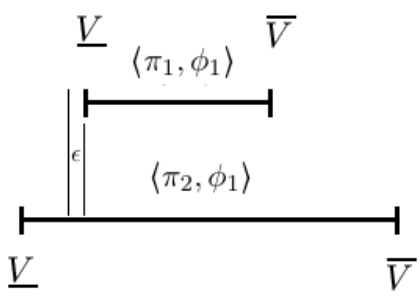

(a)

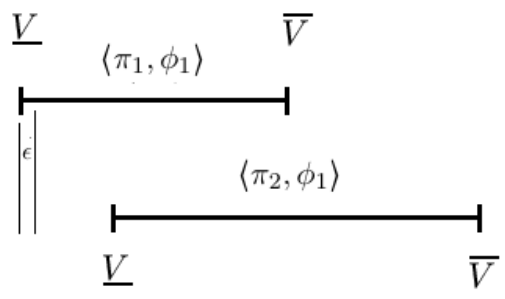

(b)

Figura 4.6: Valores das funções valor geradas pelos pares de políticas $\left\langle\pi_{1}, \phi_{1}\right\rangle$ e $\left\langle\pi_{2}, \phi_{1}\right\rangle$. Note que em todos os casos $\underline{V}\left(\pi_{1}, \phi_{1}\right)(s)-\underline{V}\left(\pi_{2}, \phi_{1}\right)(s)>\epsilon$.

Se $\underline{V}\left(\pi_{1}, \phi_{1}\right)(s)-\underline{V}\left(\pi_{2}, \phi_{1}\right)(s) \leq \epsilon$, então ambos os pares de políticas são considerados na próxima fase do algoritmo $\epsilon$-Lower-First, ou seja, $\pi_{1}(s)$ e $\pi_{2}(s) \in A_{1}[\underline{V}](s)$ e $\phi_{1}(s) \in A_{2}[\underline{V}](s)$. Assim, podem ocorrer dois casos:

- $\bar{V}\left(\pi_{1}, \phi_{1}\right)(s) \geq \bar{V}\left(\pi_{2}, \phi_{1}\right)(s)$ - nesse caso, na segunda fase do critério $\epsilon$-Lower-First, o par de políticas $\left\langle\pi_{1}, \phi_{1}\right\rangle$ é escolhido, o que corresponde a mesma escolha feita pelo critério Lower-First (Figuras 4.7(a) e 4.7(b)).

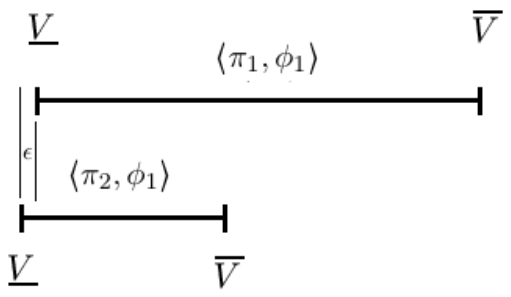

(a)

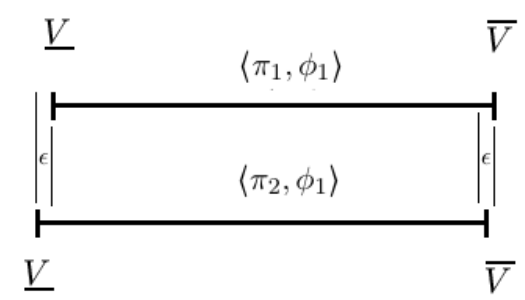

(b)

Figura 4.7: Valores das funções valor geradas pelos pares de políticas $\left\langle\pi_{1}, \phi_{1}\right\rangle$ e $\left\langle\pi_{2}, \phi_{1}\right\rangle$. Note que em todos os casos $\left|\underline{V}\left(\pi_{1}, \phi_{1}\right)(s)-\underline{V}\left(\pi_{2}, \phi_{1}\right)(s)\right| \leq \epsilon$, porém, $\bar{V}\left(\pi_{1}, \phi_{1}\right)(s) \geq \bar{V}\left(\pi_{2}, \phi_{1}\right)(s)$.

- $\bar{V}\left(\pi_{2}, \phi_{1}\right)(s)-\bar{V}\left(\pi_{1}, \phi_{1}\right)(s) \geq \epsilon$ - nesse caso, na segunda fase do critério $\epsilon$-Lower-First, o par escolhido é $\left\langle\pi_{2}, \phi_{1}\right\rangle$. Ou seja, nesse caso o algoritmo $\epsilon$-Lower-First permite um ganho maior no limite superior da função valor do que o critério Lower-First (Figura 4.8(a)). 


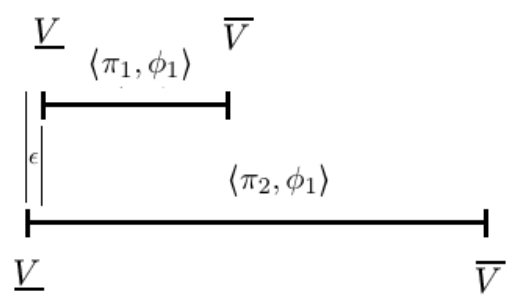

(a)

Figura 4.8: Valores das funções valor geradas pelos pares de políticas $\left\langle\pi_{1}, \phi_{1}\right\rangle$ e $\left\langle\pi_{2}\right.$, $\left.\phi_{1}\right\rangle$. Note que em todos os casos $\left|\underline{V}\left(\pi_{1}, \phi_{1}\right)(s)-\underline{V}\left(\pi_{2}, \phi_{1}\right)(s)\right| \leq \epsilon$, porém, $\bar{V}\left(\pi_{1}, \phi_{1}\right)(s)<\bar{V}\left(\pi_{2}, \phi_{1}\right)(s)$.

Assim, como analisamos todos os possíveis casos das funções valor geradas pelos pares de políticas $\left\langle\pi_{1}, \phi_{1}\right\rangle$ e $\left\langle\pi_{2}, \phi_{1}\right\rangle$, podemos garantir que o critério $\epsilon$-Lower-First sempre encontra pares de políticas com valor maior ou igual que o critério Lower-First (a menos de um $\epsilon$ com relação ao limite inferior), como queríamos demonstrar.

O Algoritmo 13 (EPSILON-LOWER-FIRST) recebe como entrada o AMG-IP dado pela tupla $\left\langle S, A_{1}\right.$, $\left.A_{2}, R, K, \gamma\right\rangle$, o número máximo de iterações (maxIter) e um parâmetro $\epsilon$ e primeiro faz uma chamada ao Algoritmo EPSILON-LOWER-ONLY de forma que os pares de políticas que alcançam $\underline{V}^{\star}(s)$ a menos de um $\epsilon$ sejam devolvidas. Em seguida o Algoritmo UPPER-ONLY é chamado para calcular $\bar{V}^{*}(s)$ e tem como resposta os pares de políticas de equilíbrio segundo a Definição 4.7 considerando $\underline{V}^{*}(s)$ empatados a menos de um $\epsilon$.

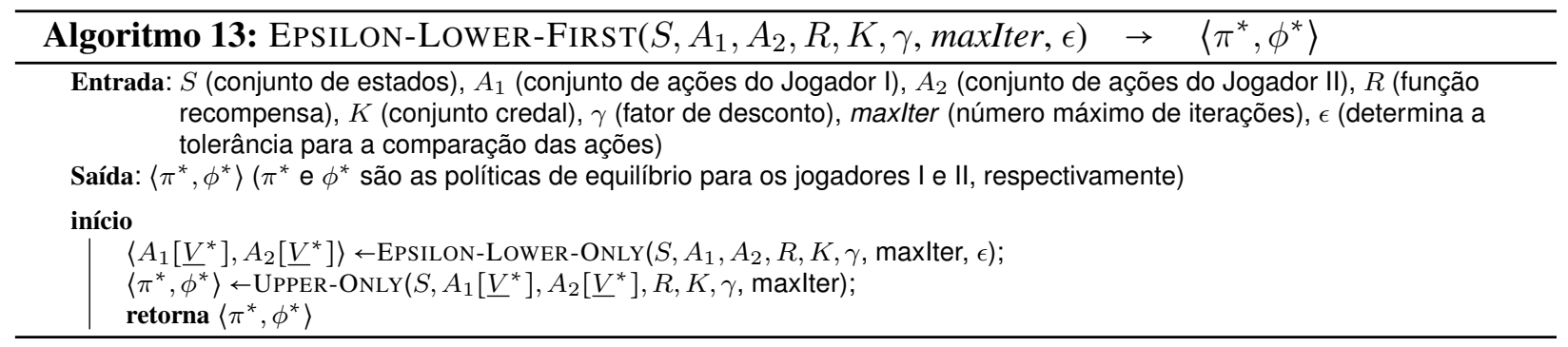

Similarmente ao critério $\epsilon$-Lower-First, podemos melhorar o critério Upper-First introduzindo um $\epsilon$ na primeira fase de execução do algoritmo. E, de forma análoga, temos o Teorema 4.4 que garante que o critério $\epsilon$-Upper-First pode melhorar as escolhas do Jogador I. A prova desse teorema é similar a prova do Teorema 4.3.

Teorema 4.4. $O$ critério $\epsilon$-Upper-First sempre encontra pares de políticas com valor maior ou igual que o critério Upper-First (a menos de um $\epsilon$ com relação ao limite superior).

E, de forma semelhante ao Algoritmo EPSILON-LOWER-FIRST, podemos construir o Algoritmo EPSILON-UPPER-FIRST que recebe como entrada o AMG-IP dado pela tupla $\left\langle S, A_{1}, A_{2}, R, K, \gamma\right\rangle$, um $\epsilon$, o número máximo de iterações (maxIter) e faz uma chamada ao Algoritmo EPSILON-UPPER-ONLY e uma chamada ao Algoritmo LOWER-ONLY. Como resposta temos os pares de políticas de equilíbrio segundo a Definição 4.8 considerando $\bar{V}^{*}(s)$ empatados a menos de um $\epsilon$. 


\subsubsection{Critério de preferência $\eta$-optimal}

Uma outra alternativa aos algoritmos Lower-First e Upper-First que considera ambos os extremos da função valor intervalar para calcular pares de políticas de equilíbrio é fazer uma combinação linear das escolhas max e min da Natureza. Assim, a função $Q\left(s, a_{1}, a_{2}\right)$ é dada por:

$$
\begin{aligned}
Q\left(s, a_{1}, a_{2}\right) & =\left[\eta\left(R\left(s, a_{1}, a_{2}\right)+\gamma \min _{p \in K\left(s, a_{1}, a_{2}\right)} \sum_{s^{\prime} \in S} p\left(s^{\prime} \mid s, a_{1}, a_{2}\right) V\left(s^{\prime}\right)\right)\right. \\
& \left.+(1-\eta)\left(R\left(s, a_{1}, a_{2}\right)+\gamma \max _{p \in K\left(s, a_{1}, a_{2}\right)} \sum_{s^{\prime} \in S} p\left(s^{\prime} \mid s, a_{1}, a_{2}\right) V\left(s^{\prime}\right)\right)\right], \\
\text { e a função } V^{*}(s) & =\max _{a_{1} \in A_{1}} \min _{a_{2} \in A_{2}} Q\left(s, a_{1}, a_{2}\right),
\end{aligned}
$$

com $\eta$, chamado de parâmetro de precaução (Utkin e Augustin 2005), variando entre 0 e 1, sendo que para $\eta=1$, temos a solução robusta (Lower-Only) e para $\eta=0$, a solução otimista (Upper-Only).

Assim, um par de políticas alcançado usando esse critério é considerado um par de políticas de equilíbrio de acordo com a Definição 4.10 .

Definição 4.10 (Par de políticas ótimas para AMG-IPs: uso de um parâmetro de precaução). Um par de políticas $\pi^{*} \in \Pi e \phi^{*} \in \Phi$ é dito estar em equilíbrio seguindo o parâmetro de precaução $\eta$ se não existe uma política $\pi \in \Pi$ tal que

$$
\eta \underline{V}\left(\pi^{*}, \phi^{*}\right)(s)+(1-\eta) \bar{V}\left(\pi^{*}, \phi^{*}\right)(s)<\eta \underline{V}\left(\pi, \phi^{*}\right)(s)+(1-\eta) \bar{V}\left(\pi, \phi^{*}\right)(s), \quad \forall s \in S,
$$

e não existe uma política $\phi \in \Phi$ tal que:

$$
\eta \underline{V}\left(\pi^{*}, \phi\right)(s)+(1-\eta) \bar{V}\left(\pi^{*}, \phi\right)(s)<\eta \underline{V}\left(\pi^{*}, \phi^{*}\right)(s)+(1-\eta) \bar{V}\left(\pi^{*}, \phi^{*}\right)(s), \quad \forall s \in S .
$$

Suponha a situação ilustrada na Figura 4.9a. Note que se usarmos os critérios Lower-Only, Lower-First ou o critério $\epsilon$-Lower-First (com $\epsilon<0.0001 \mathrm{~cm}$ ), o par de políticas escolhido seria o par $\left\langle\pi^{\prime}, \phi^{\prime}\right\rangle$, pois $\underline{V}\left(\pi^{\prime}, \phi^{\prime}\right)(s)>\underline{V}(\pi, \phi)(s)$. Porém, o par de políticas $\langle\pi, \phi\rangle$ parece uma opção melhor para o Jogador I pois o Jogador I poderá receber uma recompensa média acumulada maior para o par de políticas $\langle\pi, \phi\rangle$ do que com o par $\left\langle\pi^{\prime}, \phi^{\prime}\right\rangle$. Por outro lado, se usarmos os critérios Upper-Only, Upper-First ou $\epsilon$-Upper-First para os intervalos da Figura $4.9 \mathrm{~b}$, o par de políticas $\langle\pi, \phi\rangle$ seria o escolhido. Porém, o par $\left\langle\pi^{\prime}, \phi^{\prime}\right\rangle$ parece resultar em uma recompensa média acumulada maior.

Usando o critério $\eta$-optimal para os dois casos da Figura 4.9, com $\eta=0.5$ por exemplo, selecionamos os melhores intervalos: na situação da Figura 4.9a, o intervalo $\langle\pi, \phi\rangle$ seria o selecionado e na Figura 4.9b, o intervalo $\left\langle\pi^{\prime}, \phi^{\prime}\right\rangle$.

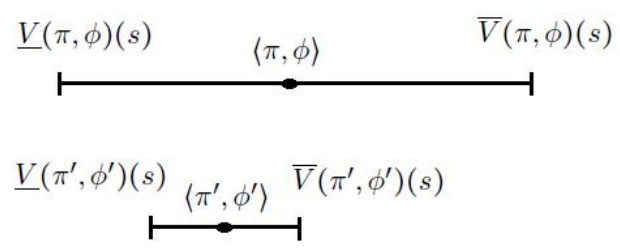

a)

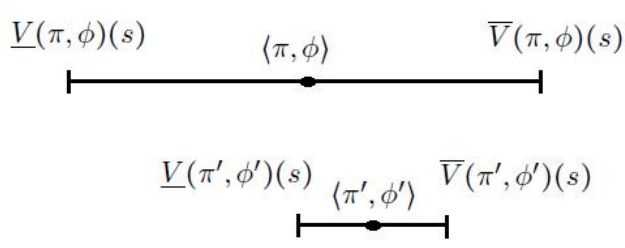

b)

Figura 4.9: Comparação entre possíveis valores da função valor gerados pelos pares de políticas $\langle\pi, \phi\rangle$ e $\left\langle\pi^{\prime}, \phi^{\prime}\right\rangle$ para $\eta=0.5$.

O Algoritmo 14 (ETA-OptIMAL) recebe como parâmetros de entrada o AMG-IP dado pela tupla $\langle S$, $\left.A_{1}, A_{2}, R, K, \gamma\right\rangle, \eta$ com valor entre 0 e 1 e um parâmetro que especifica o número máximo de iterações e tem como resposta o par de políticas de equilíbrio segundo a Definição 4.10. 


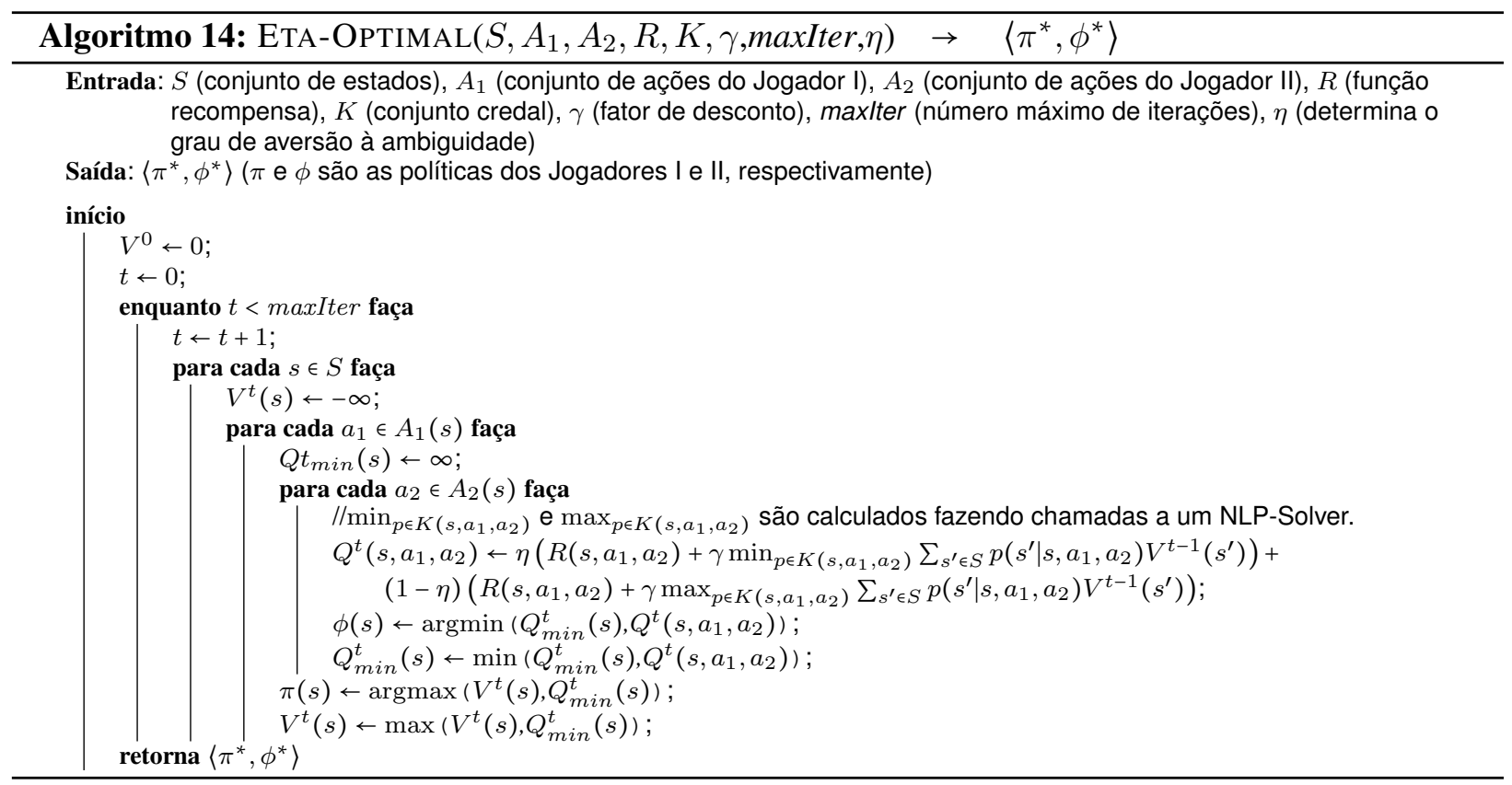

Uma questão interessante que surge com o critério $\eta$-optimal é a escolha do valor de $\eta$. Qual é o valor de $\eta$ que possibilita o Jogador I ganhar uma recompensa esperada acumulada maior?

A Figura 4.10 mostra que existe uma mudança de fase marcada pelos valores $\eta$ que faz com que para valores $\eta>\eta_{f}$, o intervalo $\left[p_{1}, q_{1}\right]$ seja considerado maior que $\left[p_{2}, q_{2}\right]$, para valores $\eta<\eta_{f}$, o intervalo maior seja $\left[p_{2}, q_{2}\right]$; e com $\eta=\eta_{f}$ os intervalos são considerados iguais. Assim, basta definir um valor para $\eta$ maior ou menor que $\eta_{f}$, dependendo do problema em questão, para definir a melhor escolha entre intervalos.

É importante notar que só há mudança de fase em intervalos do tipo "contidos".

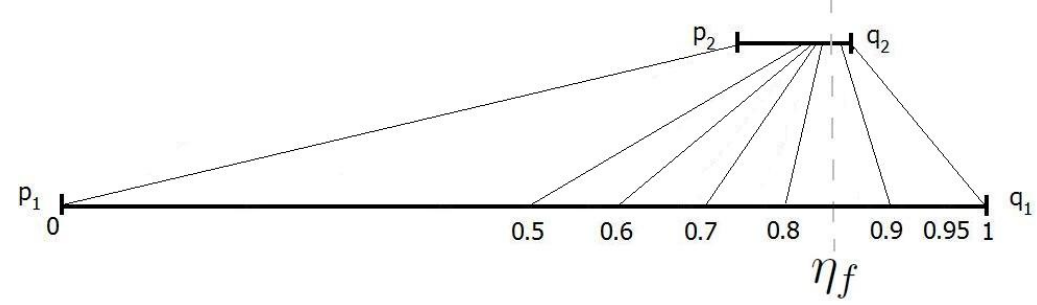

Figura 4.10: Diferentes escolhas para o parâmetro de precaução. $O$ valor de mudança de fase $\eta_{f}$ faz com que para valores $\eta>\eta_{f}$, o intervalo $\left[p_{1}, q_{1}\right]$ seja considerado maior que $\left[p_{2}, q_{2}\right]$; para valores $\eta<\eta_{f}$, o intervalo $\left[p_{2}, q_{2}\right]$ é considerado o maior; e com $\eta=\eta_{f}$ os intervalos são considerados iguais.

Uma forma de calcular o valor de $\eta_{f}$ é dada a seguir. Sejam os intervalos $\left[p_{1}, q_{1}\right]$ e $\left[p_{2}, q_{2}\right]$ representados na Figura 4.11. O valor de mudança de fase $\eta_{f}$ pode ser calculado resolvendo o seguinte sistema de equações:

$$
\begin{array}{r}
x=p_{1}+d_{1}=p_{2}+d_{2} \\
\eta_{f}\left(q_{1}-p_{1}\right)=d_{1} \\
\\
\eta_{f}\left(q_{2}-p_{2}\right)=d_{2}
\end{array}
$$

Assim, o valor de $\eta$ pode ser calculado se conhecemos a média das distâncias dos intervalos.

$$
\text { sendo } \eta_{f}=\frac{p_{1}-p_{2}}{q_{2}-p_{2}-q_{1}+p_{1}}
$$




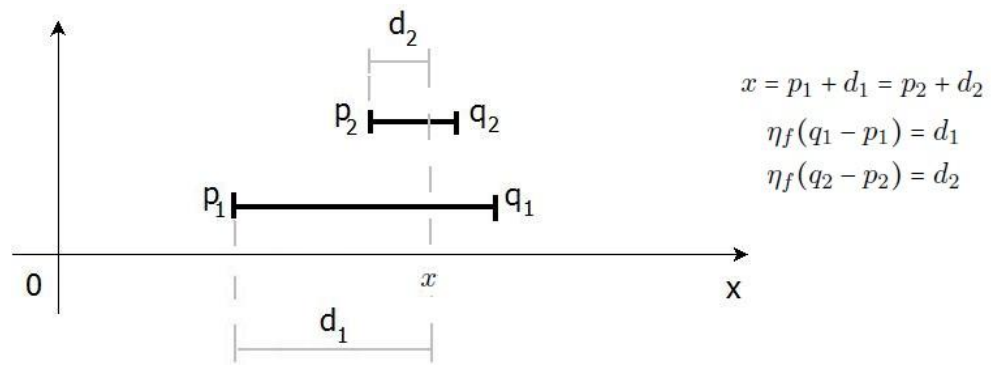

Figura 4.11: Intervalos do tipo "contidos". O eixo $x$ é usado para dimensionarmos as medidas de $p_{1}, p_{2}, q_{1}$ e $q_{2}$ com relação à origem, e as distâncias $d_{1}$ e $d_{2}$. Assumimos que exista um ponto $x$ e queremos encontrar $\eta_{f}$ tal que as restrições ao lado sejam satisfeitas.

\subsubsection{Jogo Markoviano Alternado com Transição Valorada por Conjunto - AMG-ST}

Uma outra extensão possível para as soluções discutidas nesse capítulo é considerar um caso particular de AMG-IP que chamamos de AMG-ST (inspirados no modelo MDP-ST do Capítulo 2.1).

Nesse novo modelo, a imprecisão é dada na forma de transições valoradas por conjunto, ou seja, os jogadores escolhem um par de ações a serem executadas em um estado $s \in S$ e há uma transição probabilística para um conjunto de estados $k$. De forma não-determinística é feita uma transição do conjunto $k$ para um estado $s^{\prime} \in k$. Assim, ao invés de selecionar um $K(s, p i(s), \phi(s))$ como no modelo AMG-IP, a Natureza escolhe o estado $s^{\prime} \in k$ resultante da transição de estado.

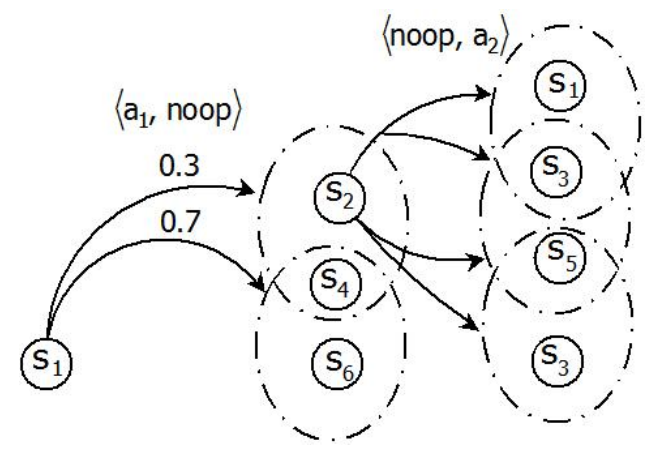

Figura 4.12: Exemplo de um AMG-ST. O par de ações $\left\langle a_{1}\right.$, noop $\rangle$ aplicado no estado $s_{1}$ levam os jogadores para o conjunto $\left\{s_{2}, s_{4}\right\}$ com probabilidade 0.3 e para o conjunto $\left\{s_{4}, s_{6}\right\}$ com probabilidade 0.7.

A Figura 4.12 ilustra um exemplo de um AMG-ST. No estado $s_{1} \in S_{1}$ o Jogador I escolhe uma ação $a_{1} \in A_{1}$ e o Jogador II uma ação noop. Ao executarem esse par de ações $\left\langle a_{1}, n o o p\right\rangle$, com probabilidade 0.3 o jogo vai para o conjunto $\left\{s_{2}, s_{4}\right\}$ e com probabilidade 0.7 para o conjunto $\left\{s_{4}, s_{6}\right\}$. A escolha do estado resultante desta transição é feita pela Natureza. Uma solução robusta para o Jogador I é considerar que a Natureza sempre escolherá um estado que resulta na menor recompensa total esperada. Assim, após a transição para o próximo estado $s^{\prime} \in\left\{s_{2}, s_{4}\right\}$ ou $s^{\prime} \in\left\{s_{4}, s_{6}\right\}$, o Jogador I executa uma ação noop e o Jogador II escolhe e executa uma ação $a_{2} \in A_{2}$ e, novamente, o estado resultante da transição depende da escolha da Natureza.

Uma solução "robusta melhorada", ou seja, adaptando o critério de preferência Lower-First da Seção 4.2.3 para AMG-STs é feita calculando as equações:

$$
\underline{V}^{*}(s)=\max _{a_{1} \in A_{1}} \min _{a_{2} \in A_{2}}\left(R\left(s, a_{1}, a_{2}\right)+\gamma \sum_{k \in F\left(s, a_{1}, a_{2}\right)} p\left(k \mid s, a_{1}, a_{2}\right) \min _{s^{\prime} \in k} \underline{V}^{*}\left(s^{\prime}\right)\right)
$$

e

$$
\bar{V}^{*}(s)=\max _{a_{1} \in A_{1}\left[\underline{V}^{*}\right](s)} \min _{a_{2} \in A_{2}\left[\underline{V}^{*}\right](s)}\left(R\left(s, a_{1}, a_{2}\right)+\gamma \sum_{k \in F\left(s, a_{1}, a_{2}\right)} p\left(k \mid s, a_{1}, a_{2}\right) \max _{s^{\prime} \in k} \bar{V}^{*}\left(s^{\prime}\right)\right),
$$


sendo $\left\langle\pi^{*}, \phi^{*}\right\rangle$ calculado por:

$$
\left\langle\pi^{*}, \phi^{*}\right\rangle=\arg \max _{a_{1} \in A_{1}\left[\underline{V}^{*}\right](s)} \min _{a_{2} \in A_{2}\left[\underline{V}^{*}\right](s)}\left(R\left(s, a_{1}, a_{2}\right)+\gamma \sum_{k \in F\left(s, a_{1}, a_{2}\right)} p\left(k \mid s, a_{1}, a_{2}\right) \max _{s^{\prime} \in k} \bar{V}^{*}\left(s^{\prime}\right)\right) .
$$

em que $A_{1}\left[\underline{V}^{*}\right](s)$ e $A_{2}\left[\underline{V}^{*}\right](s)$ são os conjuntos de ações que alcançam $\underline{V}^{*}$ e que são usadas para calcular $\bar{V}^{*}$ e $F\left(s, a_{1}, a_{2}\right)$ é a função que mapeia um estado $s$ e um par de ações $\bar{a}_{1} \in A_{1}$ e $a_{2} \in A_{2}$ para um conjunto de estados $k$. 


\section{Capítulo 5}

\section{Resultados Experimentais}

Os experimentos descritos neste capítulo não são exaustivos, mas servem para ilustrar algumas das ideias deste trabalho. Assim, nesse capítulo mostramos os resultados do uso dos algoritmos: LowERONLY, UPPER-ONLY, UPPER-FIRST, LOWER-FIRST, EPSILON-UPPER-FIRST ( $\epsilon$-Upper-First), EPSILONLOWER-FIRST ( $\epsilon$-Lower-First), ETA-OPTIMAL ( $\eta$-optimal). Comparamos os intervalos das funções valor para as políticas de equilíbrio devolvidas e avaliamos as médias da recompensa esperada acumulada por essas políticas. Para isso, foi usado um simulador de um jogo que considera a Natureza aleatória.

Os experimentos foram feitos usando o domínio do Gato e o Rato (Exemplo 1.2, Capítulo 1), em que os tamanhos dos problemas variam com grades de $3 \times 3$ até $8 \times 8$. Para os testes, consideramos que não há "barreiras" entre as células que compõem a grade do jogo (com o objetivo de aumentar o número de ações aplicáveis nos problemas pequenos e médios) e há dois buracos: um localizado nas proximidades do Jogador II, com recompensa maior, e outro nas proximidades do Jogador I, com recompensa menor. Assim, dependendo do critério de preferência escolhido, o Jogador I pode assumir uma postura mais otimista e correr um risco maior de ser pego pelo gato tentando alcançar o buraco com maior recompensa ou pode assumir uma postura mais pessimista e tentar alcançar o buraco com menor recompensa.

Mais especificamente, os dados usados nos testes são:

- Estados: $S=[\operatorname{lin} 1$, coll, lin2, col2, vez $]$, em que lin $i$ e col $i$ indicam, respectivamente, a linha e a coluna da grade do Jogador $i$ e vez indica de quem é a vez de escolher a ação. As Figuras 5.1.a e 5.1.b ilustram a grade para o jogo $3 \times 3$ e $4 \times 4$, respectivamente.

- Ações: $A_{1}=\{$ mover_para_Leste, mover_para_Oeste, mover_para_Norte, mover_para_Sul,noop $\}$ e $A_{2}=\{$ mover_para_Leste, mover_para_Oeste, mover_para_Norte, mover_para_Sul, noop $\}$, em que noop $=$ ação sem efeito.

- Recompensa: caso lin1 $=2$ e col1 $=n$ (em que $n$ é número de colunas), então Recompensa $=10$. Caso $\operatorname{lin} 1=1$ e col1 $=n-1$, então Recompensa $=100$. Caso $\operatorname{lin} 1=\operatorname{lin} 2$ e col1 $=$ col2, então Recompensa $=$ -100. Caso contrário, Recompensa $=0$.

- Função de Transição de Estados: a função de transição de estados é dada por intervalos. Suponha que o jogo esteja no estado [3,1,1,1,1] (como na Figura 5.1.a), ou seja, o Jogador I está na linha 3 coluna 1 do grid e é ele quem escolhe a ação a ser executada. Suponha agora que o Jogador I escolha a ação $\mathrm{N}$ (norte), então, com probabilidade variando entre [0.75, 0.85] o jogador faz a transição para o novo estado [2,1,1,1,2], com probabilidade variando entre [0.5, 0.15$]$ ele faz a transição para o estado $[3,2,1,1,2]$ e com probabilidade variando entre [0.5, 0.15] ele fica no estado [3,1,1,1,2]. A Figura 5.1.c ilustra como é feita essa transição. De forma semelhante atribuímos imprecisão para as outras ações do Jogador I e ações do Jogador II.

\subsection{Média da recompensa acumulada esperada}

Para saber quais critérios obtém as melhores recompensas ao longo de uma série de jogos, construímos um simulador com escolhas aleatórias da Natureza. Executando as políticas de cada critério no simulador calculamos a média da recompensa esperada acumulada e seu desvio padrão.

Note que se ao invés de usarmos um simulador com escolhas aleatórias da Natureza, assumíssemos que a Natureza se comporta de forma adversarial ao Jogador I, o critério Lower-First sempre encontraria a melhor 
recompensa esperada descontada. Se, por outro lado, a Natureza fosse cooperativa, o critério Upper-First é que alcançaria os melhores valores de recompensa esperada. Sendo assim, para não privilegiar nenhum dos critérios, optamos por uma Natureza aleatória. Dessa forma, o simulador assume que a Natureza escolhe uma distribuição aleatória de probabilidade (ou seja, uma Natureza não-estacionária).

Para garantir que o simulador faça escolhas aleatórias de distribuições de probabilidades e a partir de um conjunto credal, aplicamos o seguinte método: suponha que em um estado $s \in S$ os Jogadores I e II escolhem as ações $a_{1} \in A_{1}$ e $a_{2} \in A_{2}$. Dado que nesse trabalho definimos a imprecisão através de um conjunto de parâmetros $p_{1}, p_{2}$ e $p_{3}$ sujeitos a um conjunto de restrições $C$, queremos gerar uma distribuição $p\left(. \mid s, a_{1}, a_{2}\right)$ aleatória para ser escolhida pela Natureza. Para isso, chamamos um otimizador (solver Minos) para resolver o seguinte problema de otimização:

$$
\begin{array}{r}
\max \left(w_{1} p_{1}+w_{2} p_{2}+w_{3} p_{3}\right) \\
\text { sujeito a } C
\end{array}
$$

em que $w_{1}, w_{2}$ e $w_{3}$ são pesos escolhidos aleatoriamente e teremos como resposta do otimizador, valores para $p_{1}, p_{2}$ e $p_{3}$ que satisfazem as restrições que devido ao max, corresponde ao limite superior da região definida pelo conjunto credal. Feito isso o próximo passo é resolver o problema de otimização (chamando o Minos):

$$
\begin{array}{r}
\min \left(w_{1}^{\prime} p_{1}^{\prime}+w_{2}^{\prime} p_{2}^{\prime}+w_{3}^{\prime} p_{3}^{\prime}\right) \\
\text { sujeito a } C
\end{array}
$$

em que $w_{1}^{\prime}, w_{2}^{\prime}$ e $w_{3}^{\prime}$ são pesos escolhidos aleatoriamente e teremos como resposta do otimizador, valores para $p_{1}^{\prime}, p_{2}^{\prime}$ e $p_{3}^{\prime}$ que atendam as restrições (limite inferior da região definida pelo conjunto credal).

Assim temos duas distribuições de probabilidades que atendem as restrições, $p_{1}, p_{2}, p_{3}$ e $p_{1}^{\prime}, p_{2}^{\prime}, p_{3}^{\prime}$. Agora, escolhemos uma variável $\alpha \in[0,1]$ para fazer uma combinação linear entre os conjunto limites, ou seja:

$$
\begin{aligned}
& \alpha \frac{p_{1}+p_{1}^{\prime}}{2}+(1-\alpha) \frac{p_{1}+p_{1}^{\prime}}{2}=p_{1}^{\prime \prime} \\
& \alpha \frac{p_{2}+p_{2}^{\prime}}{2}+(1-\alpha) \frac{p_{2}+p_{2}^{\prime}}{2}=p_{2}^{\prime \prime} \\
& \alpha \frac{p_{3}+p_{3}^{\prime}}{2}+(1-\alpha) \frac{p_{3}+p_{3}^{\prime}}{2}=p_{3}^{\prime \prime}
\end{aligned}
$$

Enfim, temos que $p_{1}^{\prime \prime}+p_{2}^{\prime \prime}+p_{3}^{\prime \prime}=1$ e que tal distribuição de probabilidade é aleatória uma vez que $w_{i}$, $w_{i}^{\prime}$ e $\alpha$ são gerados de forma aleatória.

Assim, utilizando os Algoritmos LOWER-FIRST e UPPER-FIRST vistos no Capítulo 4, resolvemos o jogo do Gato e o Rato variando o tamanho das instâncias de 3x3 à 8x8. De cada instância, obtivemos um par de políticas ótimas para cada um dos algoritmos. Em seguida, executamos no simulador os pares de políticas calculados considerando o estado inicial dado pela Figura 5.1, ou seja, a posição do rato é dada por $(n, 1)$ e do gato por $(1,1)$, em que $n$ é o tamanho da instância do jogo, calculamos a média da recompensa esperada acumulada em 1000 jogos para cada um dos algoritmos.

\subsection{Comparação entre as funções valor ótimas calculadas pelos critérios Lower-First e Upper-First}

A Figura 5.2 ilustra os intervalos da função valor obtidos em cada estado usando as políticas de equilíbrio encontradas pelos critérios Lower-First e Upper-First para a instância 7x7 do jogo do Gato e o Rato. O número de estados total para esse problema é 4416. O gráfico mostra os intervalos da função valor somente para os estados 2484 até 2500.

O gráfico completo mostra que para a maioria dos estados o intervalo interno está deslocado para cima 


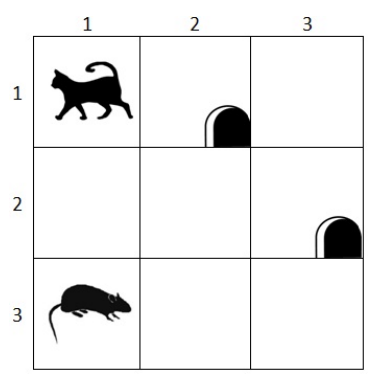

(a)

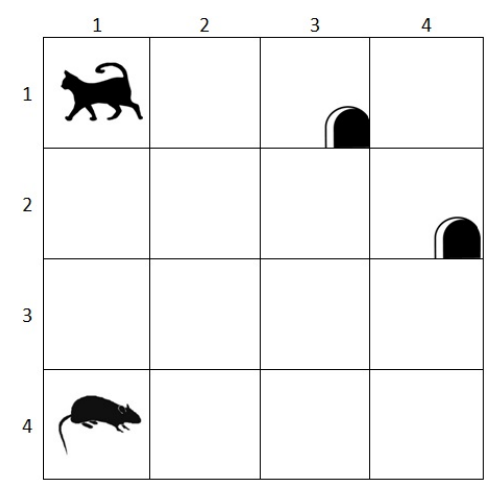

(b)

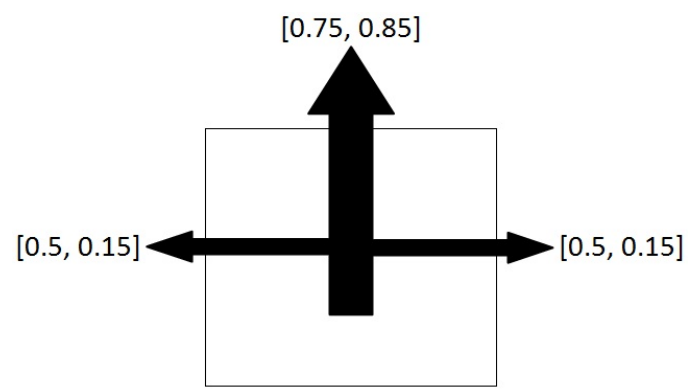

(c) Probabilidades associadas à aplicação da ação N

Figura 5.1: Em (a) e (b), ilustração de possíveis estados para os jogos $3 \times 3$ e $4 x 4$ e, em (c), ilustração da função de transição probabilística dada por intervalos.

com relação ao intervalo externo, o que indica que a solução Lower-First deve devolver uma recompensa esperada acumulada maior, como mostraremos na Seção 5.1 (Figura 5.5).

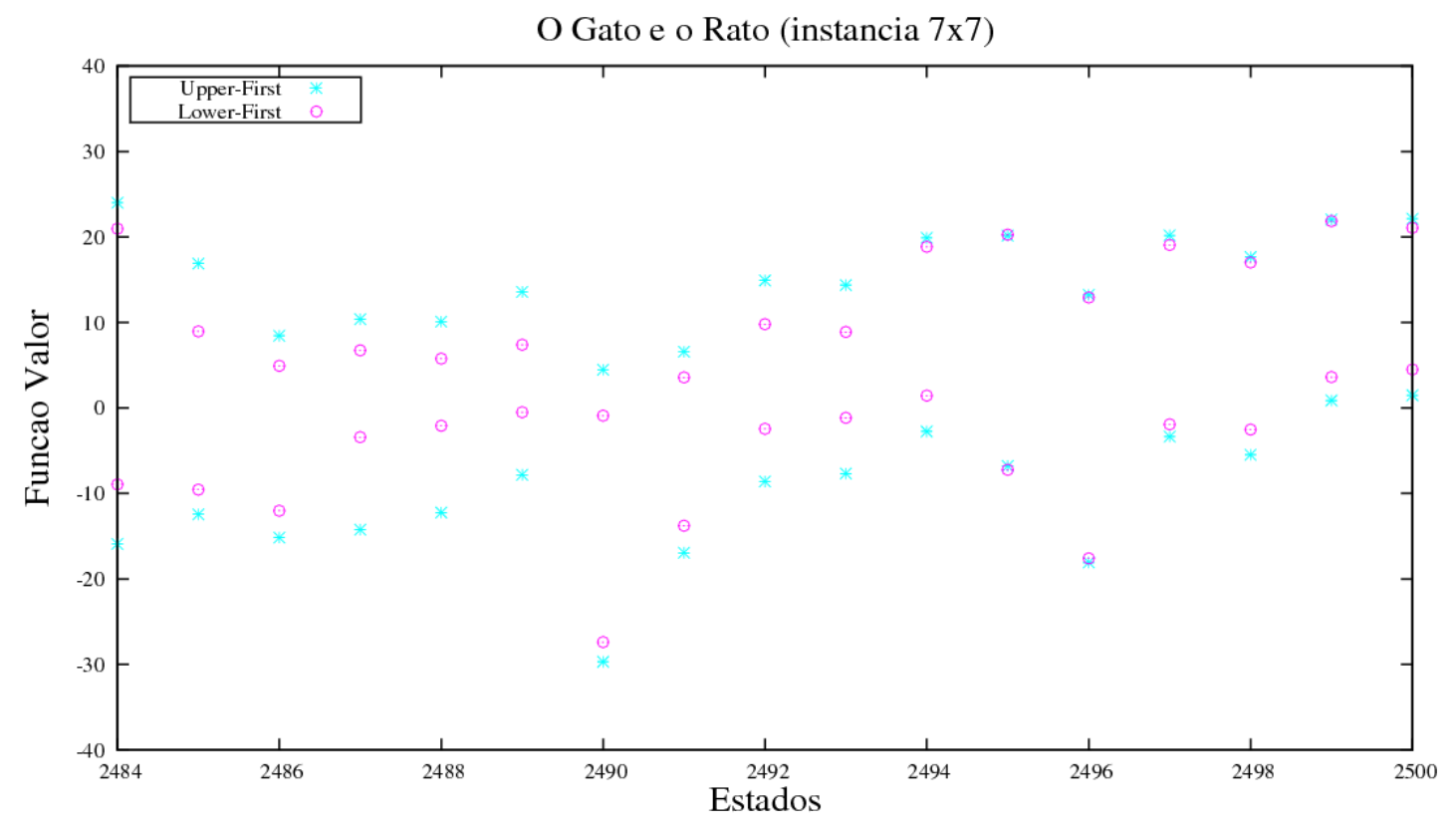

Figura 5.2: Valores intervalares da função valor alcançados pelas políticas ótimas segundo os critérios de preferência Lower-First e Upper-First para o jogo do Gato e o Rato. O número total de estados para a instância $7 x 7$ é de 4416. O gráfico mostra apenas os estados de 2484 a 2500. Note que para alguns estados as soluções coincidem enquanto para outros as soluções são diferentes indicam intervalos contidos.

Conforme discutido na Seção 4.2.5, em geral os pares de políticas encontrados pelo critério Lower-First podem ser diferentes dos pares de políticas encontrados pelo critério Upper-First, isso é devido às diferentes escolhas de ordenação (Definição 4.3 de ordenação pessimista para o critério Lower-First e Definição 4.4 de ordenação otimista para o critério Upper-First). Essa é uma das contribuições desse trabalho: mostrar que a inversão do algoritmo Upper-First proposto por Chang (2006), resultando no critério Lower-First, pode gerar políticas de equilíbrio diferentes.

Isso pode ser observado no gráfico da Figura 5.2, tanto para o conjunto de estados de 2484 a 2500 como para os demais estados (omitidos para melhorar a clareza do gráfico). Note que os intervalos com círculos nas extremidades representam a função valor para os pares de políticas devolvidos pelo Lower-First e os intervalos com ' ' ' nas extremidades representam a função valor para os pares de políticas devolvidas pelo algoritmo Upper-First. Note ainda que alguns intervalos internos estão mais deslocados para o limite superior do intervalo externo (por exemplo, os estados 2487, 2488, 2494), enquanto que outros estão mais 
deslocados para o limite inferior do intervalo externo (por exemplo, os estados 2485 e 2490). Note ainda que para os estados 2495 e 2496, ambos os critérios escolhem os mesmos intervalos.

\subsection{Comparação entre as funções valor ótimas calculadas pelos critérios $\epsilon$-Lower-First e Upper-First}

Conforme abordamos no capítulo anterior (Capítulo 4, Seção 4.2.6 e Teorema 4.3), podemos melhorar os critérios Lower-First e Upper-First introduzindo um $\epsilon$ na primeira e na segunda fase dos algoritmos. Nesta seção veremos por meio dos testes como a introdução de um $\epsilon$ melhora o critério Lower-First.

Considere os estados 2487 e 2488 da Figura 5.2 (anterior). Para esses estados o intervalo da função valor calculado com o critério Lower-First é interno ao intervalo da função valor calculada pelo critério Upper-First.

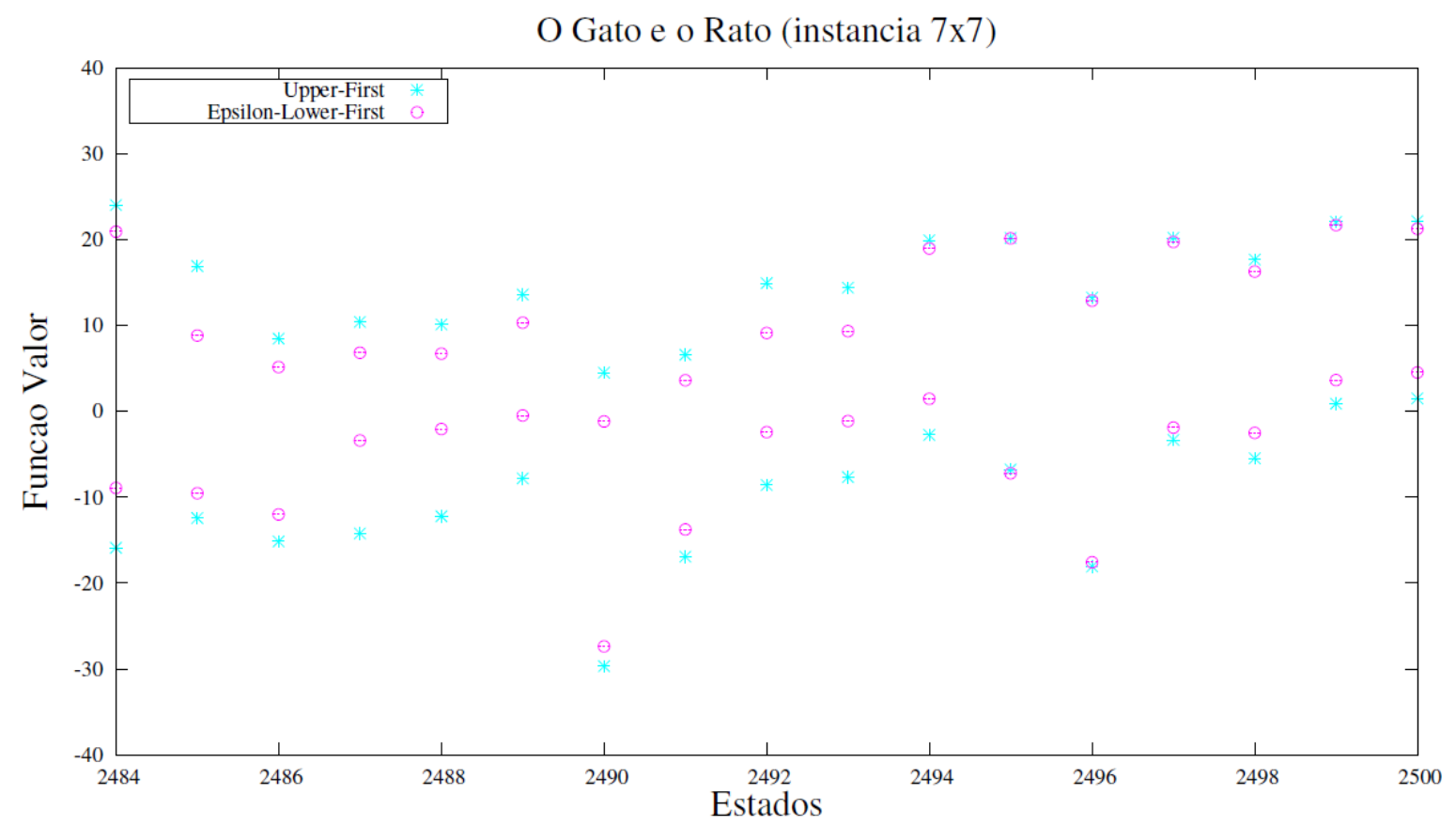

Figura 5.3: Função valor para os estados do Jogador I, seguindo as políticas ótimas geradas pelos critérios de preferência $\epsilon$-Lower-First e $\epsilon$-Upper-First para o jogo do Gato e o Rato.

A Figura 5.3 mostra que para esses estados (2487 e 2488) o critério $\epsilon$-Lower-First faz com que o limite superior $\bar{V}^{*}(s)$ seja deslocado para cima, se aproximando do limite superior do intervalo calculado pelo Upper-First. Essa aproximação se deve ao fato de que na fase 1 do algoritmo $\epsilon$-Lower-First, mais ações foram consideradas em $A_{1}\left[\underline{V}^{*}\right]$ e $A_{2}\left[\underline{V}^{*}\right]$, dada a tolerância $\epsilon$ para empate no valor $\underline{V}^{*}(s)$, possibilitando assim a seleção de um intervalo com maior limite superior.

\subsection{Comparação entre as funções valor ótimas calculadas pelos critérios $\epsilon$-Upper-First e Lower-First}

De forma análoga à comparação feita na Seção 5.3, veremos por meio dos testes como a introdução de um $\epsilon$ melhora o critério Upper-First. A Figura 5.4 mostra que para os estados 2487 e 2488, ao aplicarmos o critério $\epsilon$-Upper-First, o limite inferior $\underline{V}^{*}(s)$ é deslocado para cima. Esse resultado é justificado pelo fato de que na fase 1 do algoritmo $\epsilon$-Upper-First mais ações foram consideradas empatadas em $A_{1}\left[\bar{V}^{*}\right] \mathrm{e}$ $A_{2}\left[\bar{V}^{*}\right]$, possibilitando assim que um intervalo com maior limite inferior fosse selecionado.

\subsection{Comparação entre a média da recompensa acumulada para os critérios Lower-First, Upper-First, $\epsilon$-Lower-First e $\epsilon$-Upper-First}

O gráfico da Figura 5.5 mostra a média da recompensa acumulada para as instâncias $n \times n$, com $n$ variando de 3 a 8, usando os algoritmos Upper-First e Lower-First. A explicação para as recompensas maiores para o algoritmo Lower-First é que, a maioria dos intervalos escolhidos por esse critério está mais deslocado para valores maiores de recompensa. 


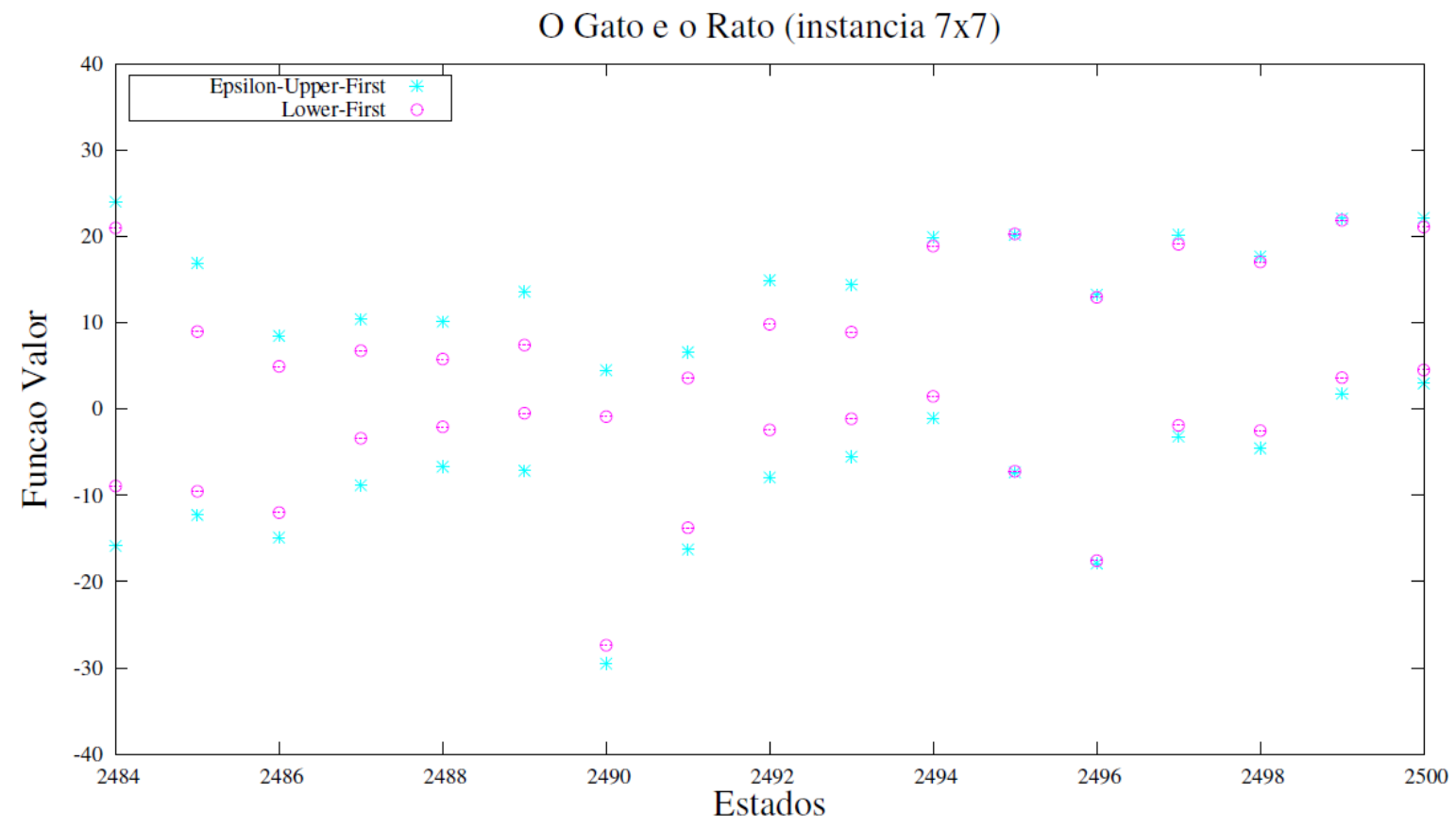

Figura 5.4: Valores intervalares da função valor alcançados pelas políticas ótimas segundo os critérios de preferência $\epsilon$-Lower-First e $\epsilon$-Upper-First para o jogo do Gato e o Rato.

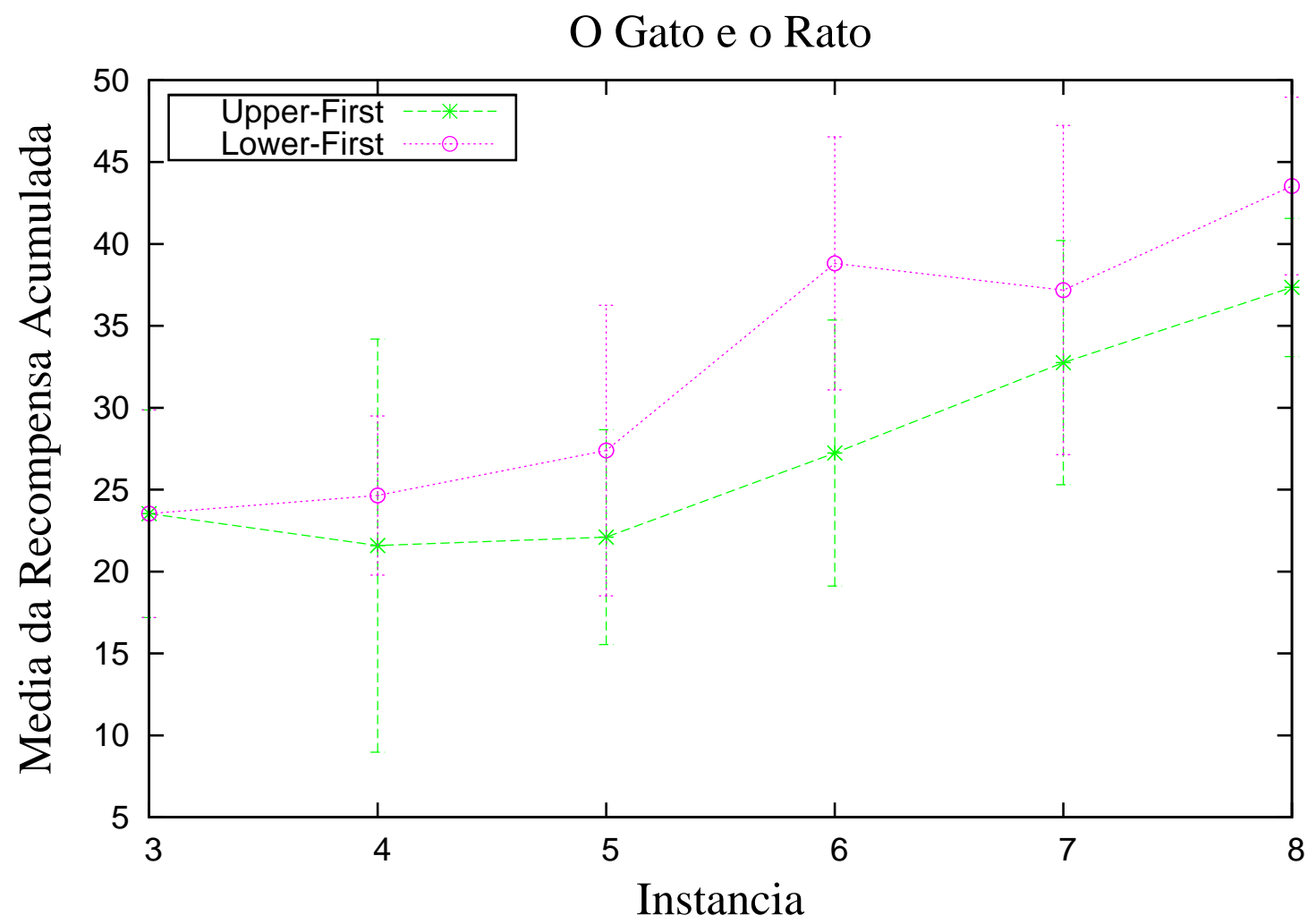

Figura 5.5: Média da recompensa acumulada esperada, no estado $s_{0}$, obtida pelos critérios Lower-First e UpperFirst, simulando uma Natureza aleatória.

A Figura 5.6 mostra que na maioria das instâncias, os pares de políticas encontrados pelo algoritmo EPSILON-UPPER-FIRST resultam em uma recompensa esperada acumulada maior do que os pares de políticas encontrados pelo algoritmo EPSILON-LOWER-FIRST. Isso mostra que com os critérios $\epsilon$-Upper-First e $\epsilon$-Lower-First ocorreram empates na primeira fase que implicaram em uma escolha melhor na segunda 
fase (favorecendo ainda mais o critério $\epsilon$-Upper-First).

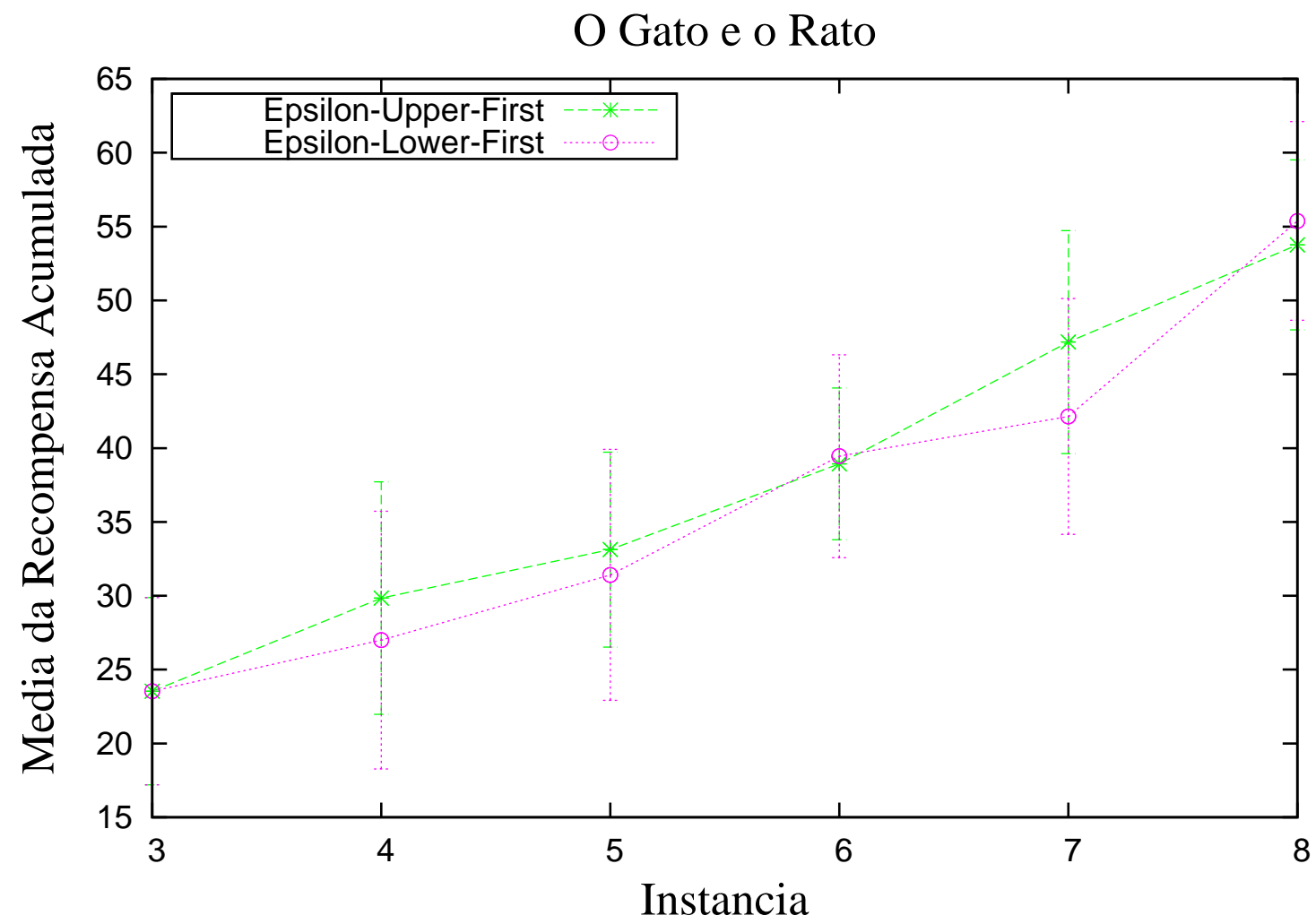

Figura 5.6: Média da recompensa acumulada esperada, no estado $s_{0}$, obtida pelos critérios $\epsilon$-Lower-First e $\epsilon$-UpperFirst simulando uma Natureza aleatória.

Em suma, embora o critério Lower-First alcance uma média da recompensa esperada acumulada maior do que o critério Upper-First (como mostrado na Figura 5.5) ao considerarmos um $\epsilon$ no momento de calcular a primeira fase de ambos os critérios, obtemos políticas de equilíbrio que resultaram em uma média de recompensa esperada acumulada maior, sendo o critério $\epsilon$-Upper-First o que alcança os maiores valores (Figura 5.7).

\subsection{Função valor e média da recompensa acumulada esperada usando o critério $\eta$-Optimal}

Uma outra forma de melhorar os critérios Lower-Only e Upper-Only (além dos critérios Lower-First, $\epsilon$-Lower-First, Upper-First e $\epsilon$-Upper-First), é utilizar um parâmetro $\eta$ que consiste em uma combinação linear dos limites superiores e inferiores da função valor intervalar.

A partir da Figura 5.8 podemos notar que os valores da função valor alcançados pelo critério $\eta$-Optimal para a instância 3x3 do jogo do Gato e o Rato, sempre estão acima dos valores alcançados pelo critério Lower-Only e abaixo dos valores alcançados pelo critério Upper-Only. Isso se deve ao fato de que o $\eta$-Optimal é uma combinação linear entre os dois extremos do intervalo da função valor intervalar. Por exemplo, ao assumir um $\eta=0.25$, assume-se uma posição um mais otimista do que o critério Lower-Only, mas não tão otimista quanto o critério Upper-Only. Ao considerar um $\eta=0.75$ assume-se uma posição mais pessimista do que o critério Upper-Only mas não tão pessimista quanto o critério Lower-Only e com um $\eta=0.50$ é feita uma suposição considerando o mesmo risco de uma posição otimista e pessimista. Como em geral, não podemos garantir qual dos critérios, Lower-Only ou Upper-Only, alcançarão melhores valores da função valor, o critério $\eta$-Optimal pode ser um bom critério, com um ajuste correto do valor de $\eta$.

A Figura 5.9 ilustra os gráficos da média de recompensa esperada acumulada obtidos considerando a solução ETA-OPTIMAL ( $\eta$-Optimal) variando o tamanho das instâncias do jogo do Gato e o Rato de 3x3 à $8 \mathrm{x} 8$ e usando valores de $\eta=0.25, \eta=0.5$ e $\eta=0.75$. Para cada instância, calculamos um par de políticas ótimas para cada um dos diferentes valores de $\eta$. Em seguida, utilizando o mesmo estado inicial usado nos gráficos anteriores, calculamos a média da recompensa esperada acumulada em 1000 jogos. Como 


\section{O Gato e o Rato}

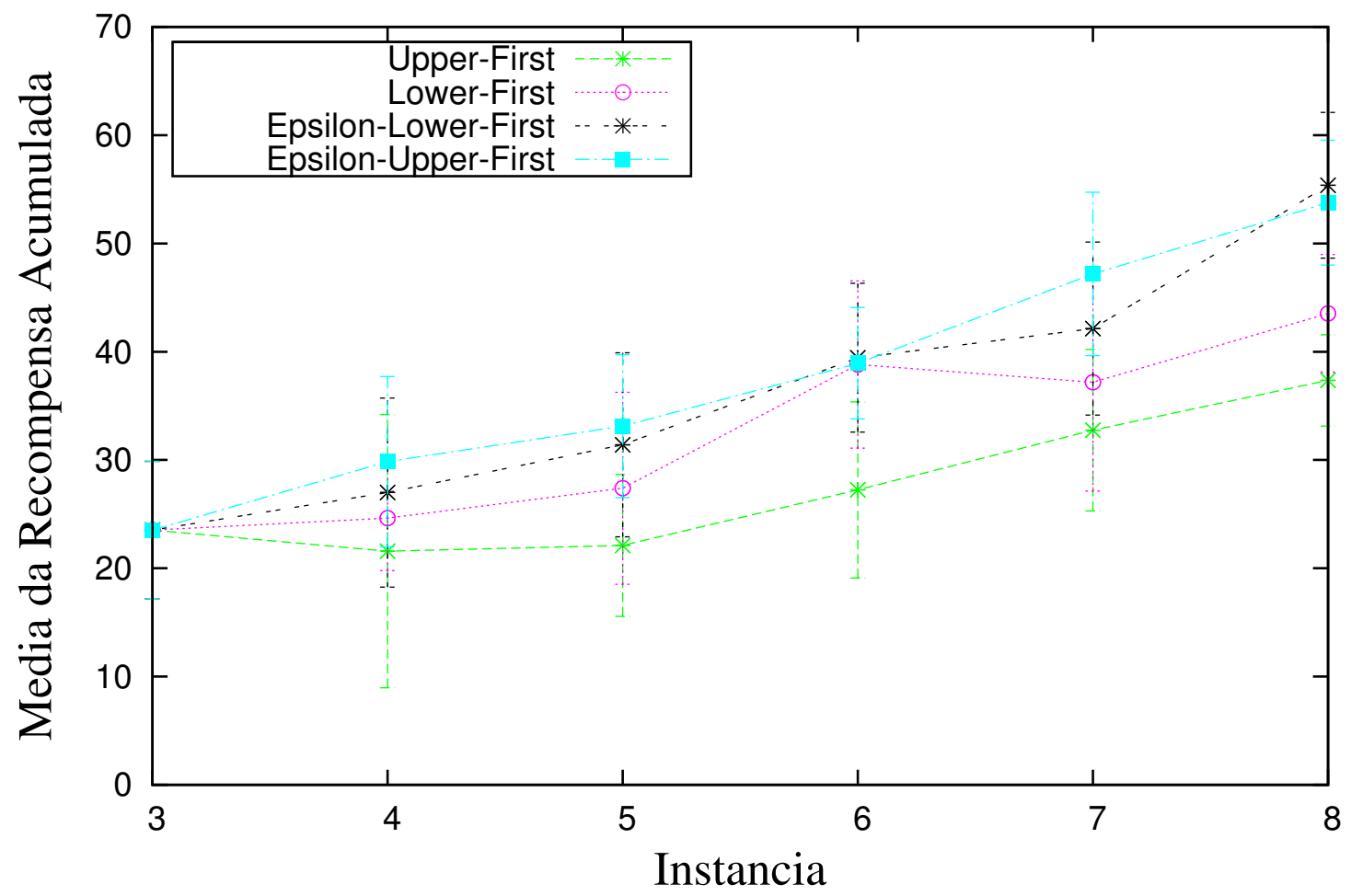

Figura 5.7: Média da recompensa esperada acumulada pelas políticas ótimas encontradas pelos critérios de preferência Lower-First, Upper-First, $\epsilon$-Lower-First e $\epsilon$-Upper-First para o jogo do Gato e o Rato.

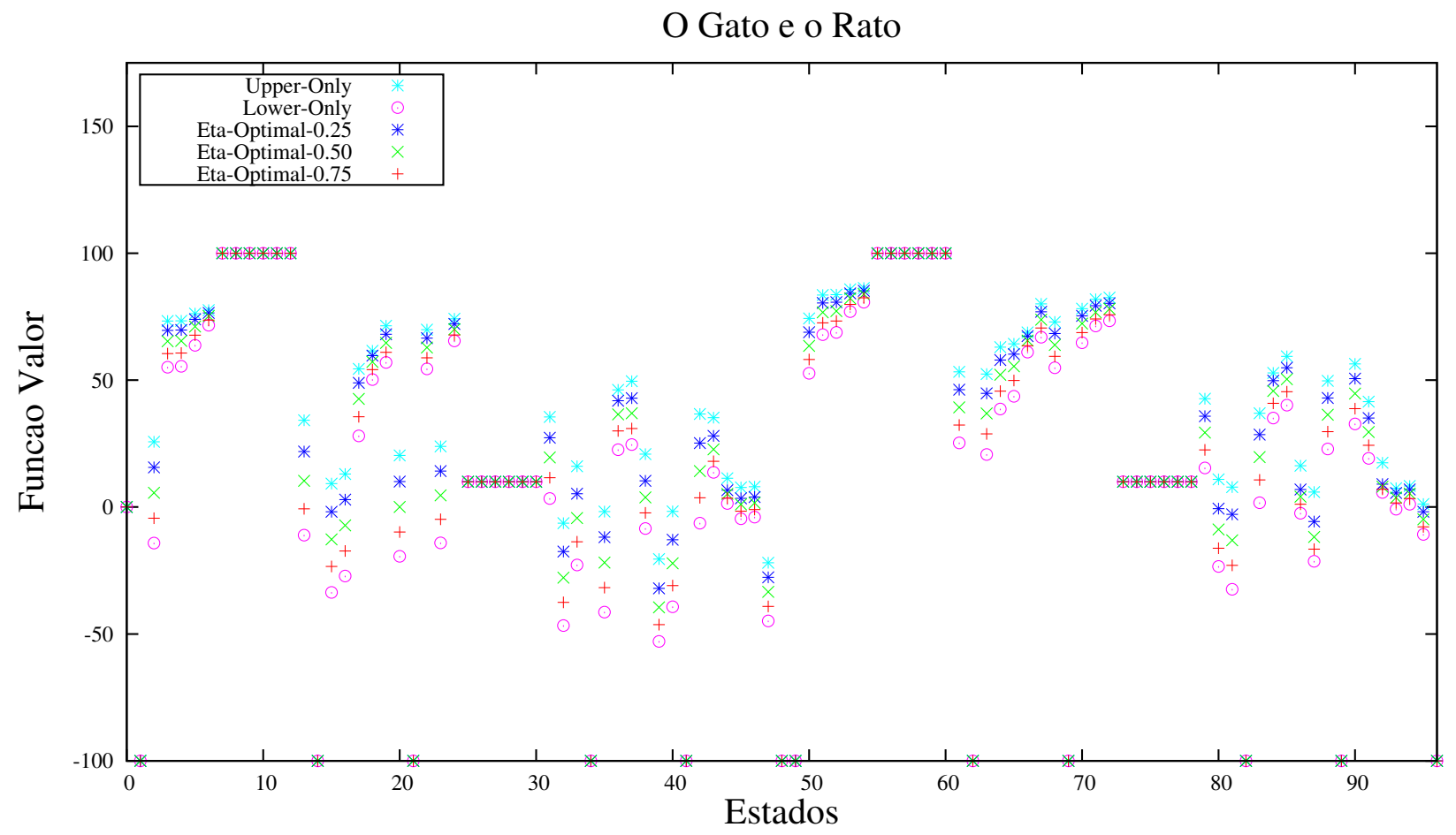

Figura 5.8: Função valor das políticas ótimas devolvidas pelos critérios de preferência Lower-Only, Upper-Only e $\eta$-Optimal para valores de $\eta=\{0.25,0.50,0.75\}$ na instância $3 x 3$ do jogo do Gato e o Rato.

resultado, obtivemos o gráfico ilustrado pela Figura 5.9.

Na Figura 5.9, um valor $\eta=0.75$ alcançou uma média de recompensa esperada acumulada maior em 


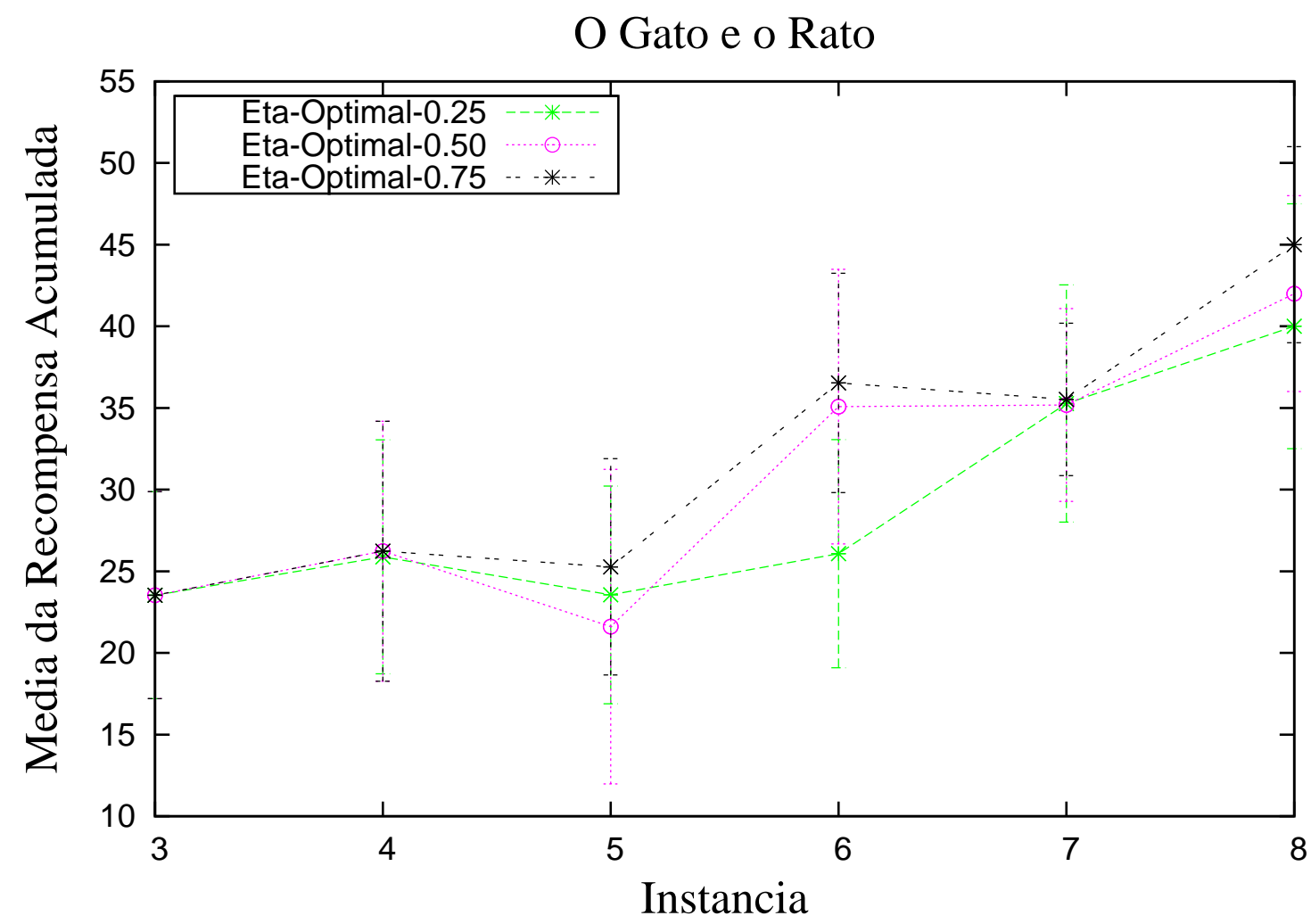

Figura 5.9: Média da recompensa acumulada esperada obtida pelo critério $\eta$-Optimal em que $\eta$ é 0.25 , 0.5 ou 0.75 , simulando uma Natureza aleatória.

todas as instâncias em comparação com outros valores de $\eta$. Isso indica que para o jogo do Gato e o Rato, uma postura mais otimista garante em média um valor de recompensa esperada acumulada maior quando a Natureza é aleatória.

Finalmente, o gráfico da Figura 5.10 compara três soluções propostas nesse trabalho. Note que o algoritmo EPSILON-UPPER-FIRST obteve, em média, maiores recompensas esperadas acumuladas do que os algoritmos EPSILON-LOWER-FIRST e ETA-OPTIMAL com $\eta=0.75$.

\subsubsection{Comparação entre os tempos de execução dos critérios}

A Figura 5.11 ilustra os tempos gastos para os critérios Lower-First, Upper-First, $\epsilon$-Lower-First, $\epsilon$ Upper-First e $\eta$-Optimal, em que $\eta=0.75$ para as mesmas instâncias analisadas anteriormente $(3 \times 3$ à $8 \times 8)$. Podemos notar que em geral o tempo gasto é exponencial ao tamanho do problema.

O critério $\eta$-Optimal (ETA-OPTIMAL) mostrou um tempo gasto maior do que todos os outros critérios analisados, isso se deve ao fato de que em cada iteração e para cada tripla 〈estado, ação do Jogador I, ação do Jogador II $\rangle$, o critério $\eta$-Optimal realiza duas chamadas ao NPL-Solver, enquanto que os outros critérios, em geral, só fazem uma chamada (no pior caso podem chegar a fazer o mesmo número de chamadas ao NPL-Solver que o critério $\eta$-Optimal, no caso em que todas as ações empatam na primeira fase). Podemos notar também que os critérios Lower-First e Upper-First mostraram um tempo gasto um pouco menor do que os critérios Epsilon-Lower-First, Epsilon-Upper-First. Isso se deve ao fato de que não há muitos (ou nenhum) empate na primeira fase dos critérios Lower-First e Upper-First, o que faz com que a segunda fase do algoritmo convirja muito rápido. Sabemos também que a parte mais custosa dos critérios são as chamadas feitas ao solver. No caso dos critérios Lower-First, Upper-First, Epsilon-Lower-First e Epsilon-Upper-First essa chamada é feita uma vez a cada iteração. Ao considerar empates com um $\epsilon$, várias ações empatam na primeira fase e, portanto, a segunda fase também é um pouco custosa. Já o critério $\eta$-Optimal chama o solver pelo menos duas vezes em cada iteração, o que o deixa muito custoso. Finalmente, os critérios Lower-Only e Upper-Only apresentam um tempo gasto menor por realizarem apenas uma chamada ao solver a cada iteração, em cada estado, para todos os pares de ações dos jogadores. 


\section{O Gato e o Rato}

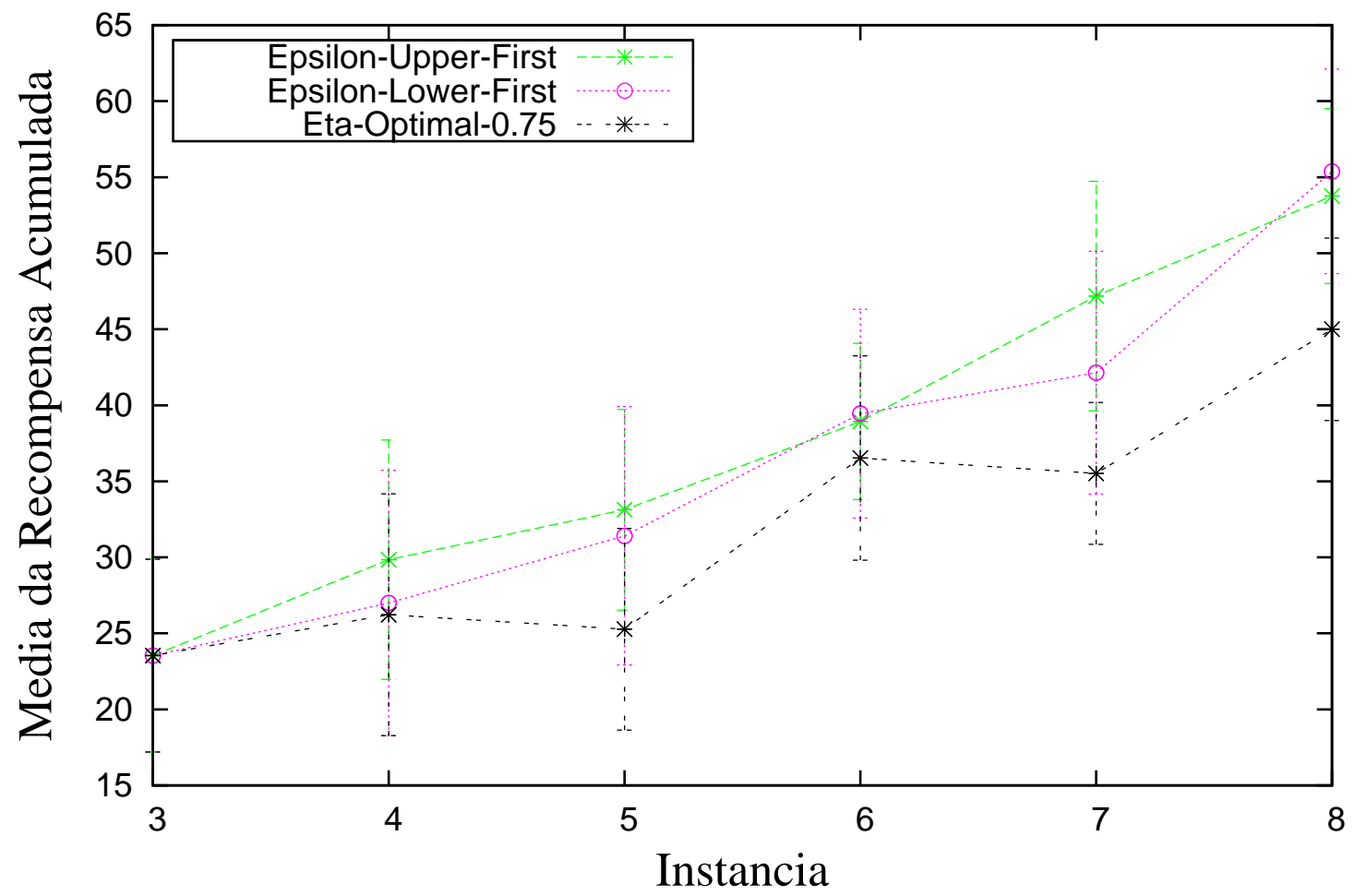

Figura 5.10: Média da recompensa acumulada esperada obtida pelos critérios $\epsilon$-Lower-First, $\epsilon$-Upper-First e $\eta$ Optimal, em que $\eta=0.75$, simulando uma Natureza aleatória, para instâncias $n \times n$ com $n$ variando de 3 a 8 .

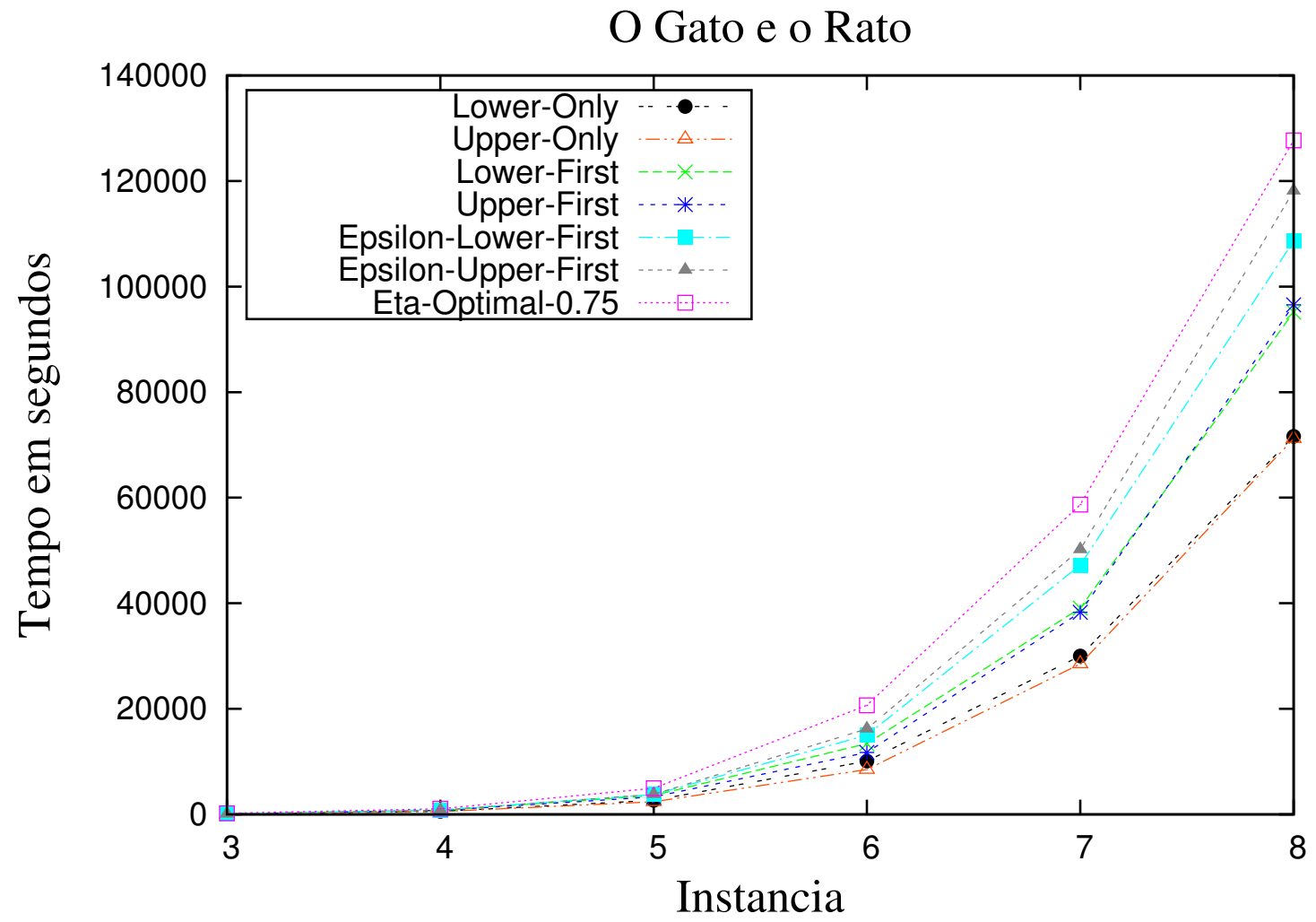

Figura 5.11: Tempo gasto pelos critérios Lower-First, Upper-First, $\epsilon$-Lower-First, $\epsilon$-Upper-First e $\eta$-Optimal, em que $\eta=0.75$ para instâncias $n \times n$ com $n$ variando de 3 a 8 . 
Assim, podemos concluir que o fator decisivo para diminuir o tempo gasto na execução dos algoritmos que implementam os critérios discutidos neste trabalho é diminuir o número de chamadas ao NPL-Solver, como havíamos citado anteriormente. 


\section{Capítulo 6}

\section{Conclusões e Trabalhos Futuros}

\subsection{Conclusão}

A teoria dos jogos interpreta qualquer ambiente multiagente como um jogo, desde que o impacto de cada agente sobre os outros seja significativo, independentemente dos agentes serem cooperativos ou competitivos [Russell e Norvig (2010)]. Assim, este trabalho contribui principalmente com a área de sistemas multiagentes, visto que, foram feitas propostas de generalizações de AMGs, a saber:

1. AMG-ST-1 (Alternating Markov Game with Set-valued Transitions for player 1): que permite modelar um dos jogadores como a Natureza (Capítulo 3). Mostramos a equivalência entre um MDP-ST e um jogo markoviano alternado de soma zero, em que a escolha não determinística entre os elementos dos conjuntos de estados alcançáveis (escolhas da Natureza) pode ser modelada como um jogador adversarial com ações determinísticas. Como resultado, obtemos uma nova classe de AMGs, em que as ações de um jogador o leva para um conjunto de estados, enquanto que as ações do outro jogador o leva para elementos desses conjuntos (de acordo com uma distribuição de probabilidades).

2. AMG-IP (Alternating Markov Game with Imprecise Probabilities): que permite que a imprecisão nas probabilidades de transição de estados seja dada por conjuntos credais (Capítulo 4). Esse modelo pode ser visto como uma extensão de um Processo de Decisão Markoviano com Probabilidades Imprecisas (Markov Decision Process with Imprecise Probabilities - MDP-IP) para ambientes multiagentes e como uma generalização do AMG-INTERVAL proposto por Chang (2006). Também mostramos as provas de convergência para o novo modelo e discutimos as diversas soluções que alcançam um valor de equilíbrio para AMGs com probabilidades imprecisas (dadas por restrições sobre parâmetros ou intervalos) sendo que cada solução depende do critério de preferência dos jogadores diante das escolhas da Natureza, ou seja, dado que a Natureza é a responsável pela imprecisão das probabilidades, cada suposição sobre o comportamento da Natureza implica em uma solução diferente. Entre as soluções para AMG-IPs propostas neste trabalho temos:

- Upper-First: "dentre os pares de políticas ótimas de equilíbrio supondo a Natureza cooperativa, escolhemos um par de políticas de equilíbrio assumindo agora a Natureza adversarial". Esse foi o critério proposto por Chang (2006), porém, adotamos uma definição de equilíbrio diferente e o comparamos a um novo critério similar: o Lower-First.

- Lower-First: "dentre os pares de políticas ótimas de equilíbrio supondo a Natureza adversarial, escolhemos um par de políticas de equilíbrio assumindo agora a Natureza cooperativa". Esse é um critério robusto e provamos que os pares de políticas encontrados por esse critério estão em equilíbrio. Com essa nova solução mostramos uma variação importante do trabalho de Chang (2006). Comparamos essa solução com o critério Upper-First e verificamos que esses dois critérios podem devolver pares de políticas de equilíbrio diferentes.

- $\epsilon$-Lower-First e $\epsilon$-Upper-First: esses critérios permitem uma tolerância $\epsilon$ na primeira fase das soluções Lower-First e Upper-First e assim, é possível obter uma recompensa média esperada maior que a solução de Chang (2006) (Upper-First), e o critério Lower-First. Assim, provamos que os critérios $\epsilon$-Lower-First e $\epsilon$-Upper-First encontram políticas melhores ou iguais que os pares de políticas encontrados pelos critérios Lower-First e Upper-First, respectivamente. 
- $\eta$-optimal: esse critério de preferência permite uma maior flexibilidade sobre as suposições de escolhas da Natureza, considerando uma combinação linear entre os limites inferiores e superiores dos intervalos da função valor. Dependendo do valor assumido por um parâmetro $\eta$, chamado de parâmetro de precaução, que pode variar entre 0 e 1 , o jogador pode definir uma medida de equilíbrio entre uma suposição mais otimista ou pessimista sobre o comportamento da Natureza. O algoritmo proposto usando esse critério, determina uma função real (não intervalar).

Além disso, na Seção 4.2.8, propomos um novo modelo de AMG com imprecisão, o AMG-ST (Alternating Markov Games with Set-Valued Transition), que é uma classe especial de AMG-IP em que as transições (para ambos os jogadores) são dadas para conjuntos de estados, sendo aplicáveis todas as soluções anteriores propostas para AMG-IPs.

Finalmente, no Capítulo 5, descrevemos os experimentos realizados para o jogo do Gato e o Rato, comparando os diferentes critérios de escolha da Natureza para soluções de AMG-IP, que confirmam os resultados teóricos discutidos no Capítulo 4. No domínio estudado mostramos que o critério Lower-First alcança valores de recompensa esperada maiores do que o critério Upper-First. Por outro lado, ao considerar um pequeno valor de $\epsilon$ (cerca de 1\% da maior recompensa) o critério Epsilon-Upper-First alcança maiores valores de recompensa esperada do que o critério Epsilon-Lower-First e os critérios Lower-First e UpperFirst. Assim, mostramos que ao considerar um $\epsilon$ na primeira fase dos algoritmos propostos é possível obter políticas de equilíbrio com melhores resultados, mesmo quando o comparamos à solução $\eta$-optimal, sendo esse resultado uma das principais contribuições desse trabalho.

\subsection{Trabalhos futuros}

Dentre as possíveis extensões propostas como trabalhos futuros estão:

- testar os critérios de preferência discutidos neste trabalho em novos problemas. Dessa forma, poderemos entender melhor quais são as propriedades de um dado domínio que contribuem para que uma solução seja melhor do que a outra;

- propor soluções eficientes para AMG-IPs baseadas em modelos fatorados e programação dinâmica em tempo real. Entre outras coisas, isso possibilitaria testar instâncias ainda maiores e contribuiria para o entendimento do comportamento dos diferentes critérios;

- aplicar os diferentes critérios de preferência propostos para AMGs propondo novas soluções para MDP-STs, BMDPs e MDP-IPs;

- investigar diferentes problemas e comparar os diferentes critérios de preferência para o modelo AMGST. Assim como o estudo feito neste trabalho para AMG-IPs, podemos aplicar os mesmos critérios para o modelo AMG-ST, com a vantagem de que para resolver um AMG-ST não necessitamos fazer chamadas ao solver, o que reduz sua complexidade. 


\section{Referências Bibliográficas}

Altman et al.(1996) E. Altman, E. A. Feinberg e A. Shwartz. Weighted discounted stochastic games with perfect information. Em CC Pub., Electrical Engineering, volume 146, páginas 18-31. Citado na pág. 5

Barto et al.(1995) A.G. Barto, S.J. Bradtke e S.P. Singh. Learning to act using real-time dynamic programming. Artificial Intelligence, 72(1-2):81-138. Citado na pág. 2, 10, 12

Bewley e Kohlberg(1978) T. Bewley e E. Kohlberg. On stochastic games with stationary optimal strategies. Em Mathematics of Operations Research, volume 3, páginas 104-125. INFORMS. Citado na pág. 5

Bonet e Geffner(2003) B. Bonet e H. Geffner. Labeled RTDP: Improving the convergence of real-time dynamic programming. Em International Conference on Automated Planning and Scheduling (ICAPS), páginas 12-21. AAAI Press. Citado na pág. 1, 2, 10

Boutilier et al.(1999) C. Boutilier, S. Hanks e T. Dean. Decision-theoretic planning: Structural assumptions and computational leverage. Journal of Artificial Intelligence Research, 11:1-94. Citado na pág. 1

Buffet e Aberdeen(2005) O. Buffet e D. Aberdeen. Robust planning with LRTDP. Em In Proc. of the 19th Int. Joint Conf. on Artificial Intelligence (IJCAI), páginas 1214-1219. Citado na pág. 12

Chang(2006) H. S. Chang. Perfect information two-person zero-sum Markov games with imprecise transition probabilities. Em Mathematical Methods of Operations Research, páginas 335-351. Springer-Verlag. Citado na pág. xv, 3, 4, 5, 11, 17, 28, 29, 30, 31, 32, 39, 41, 42, 53, 61

Cozman(2000) F. G. Cozman. Credal networks. Em AI Journal, volume 120, páginas 199-233. Citado na pág. $14,15,27,29$

Dai e Goldsmith(2007) P. Dai e J. Goldsmith. Multi-Threaded BLAO* Algorithm. Em Proceedings of the Twentieth International Florida Artificial Intelligence Research Society Conference - FLAIRS, páginas 56-61. Citado na pág. 2

de Barros et al.(2012) L. N. de Barros, F. O. Franco e K. V. Delgado. Processo de de decisão markoviano com transição valorada por conjunto modelado como um jogo alternado de soma zero. Em IX Encontro Nacional de Inteligência Artificial (ENIA). Citado na pág. 21, 27

Delgado(2010) K. V. Delgado. Processos de decisão markovianos fatorados com probabilidades imprecisas. Tese de Doutorado, Instituto de Matemática e Estatística - USP, São Paulo, SP. Citado na pág. 1, 2, 3, 7 , 10,14

Delgado et al.(2009) K. V. Delgado, S. Sanner, L. N. de Barros e F. G. Cozman. Efficient solutions to factored MDPs with imprecise transition probabilities. Em International Conference on Automated Planning and Scheduling. Citado na pág. 15

Delgado et al.(2011) K. V. Delgado, S. Sanner e L. N. de Barros. Efficient solutions to factored MDPs with imprecise transition probabilities. Em AI Journal. Accepted 03 January 2011. Citado na pág. 4, 5, 15

Feng e Hansen(2002) Z. Feng e E. A. Hansen. Symbolic heuristic search for factored Markov decision processes. Em In Proceedings of the Eighteenth National Conference on Artificial Intelligence, páginas 455-460. Citado na pág. 2 
Franco et al.(2011) F. O. Franco, L. N. de Barros, K. V. Delgado e F. G. Cozman. Jogos markovianos alternados com probabilidades imprecisas dadas por conjuntos credais. Em Anais do XXXI Congresso Sociedade Brasileira de Computação, páginas 630-641. Citado na pág. 27

Givan et al.(1997) R. Givan, S. Leach e T. Dean. Bounded parameter Markov decision processes. Artificial Intelligence, 122:234-246. Citado na pág. 5, 12

Givan et al.(2000) R. Givan, S. Leach e T. Dean. Bounded-parameter Markov decision processes. Em AI Journal, páginas (122)71-109. Elsevier. Citado na pág. 5, 8, 9, 10, 11, 31, 32, 35, 39

Guestrin(2003) C. Guestrin. Planning under uncertainty in complex structured environments. Tese de Doutorado, Stanford University. Adviser-Daphne Koller. Citado na pág. 1

Hansen e Zilberstein(1999) E. A. Hansen e S. Zilberstein. Solving Markov decision problems using heuristic search. Relatório técnico, Association for the Advancement of Artificial Intelligence - AAAI. Citado na pág. 2

Howard(1960.) R. A. Howard. Dynamic Programming and Markov Process. The MIT Press, Cambridge, Massachusetts (United States). Citado na pág. 7

Huang et al.(2004) M. Huang, R. P. Malhamé e P. E. Caines. On a class of large-scale cost-coupled Markov games with applications to decentralized power control. Em 43rd IEEE Conference on Decision and Control, páginas 2830-2835. Citado na pág. 5

Kearns et al.(2000) M. Kearns, Y. Mansour e S. Singh. Fast planning in stochastic games. Em In Proceedings of Conference on Uncertainty in Artificial Intelligence (UAI) - 2000, páginas 309-316. Morgan Kaufmann. Citado na pág. 5

Kolobov et al.(2012) A. Kolobov, P. Dai, Mausam e D. S. Weld. Reverse iterative deepening for finitehorizon MDPs with large branching factors. Em Lee McCluskey, Brian Williams, José Reinaldo Silva e Blai Bonet, editors, ICAPS. AAAI. Citado na pág. 10

Kurano et al.(1998) M. Kurano, J. Song, M. Hosaka e Y. Huang. Controlled Markov set-chains with discounting. Em J. Appl. Prob., páginas (35)293-302. Citado na pág. 5, 11

Lagoudakis e Parr(2002) M. G. Lagoudakis e R. Parr. Value function approximation in zero-sum Markov games. Em In Proceedings of the 18th Conference on Uncertainty in Artificial Intelligence (UAI 2002, páginas 283-292. Morgan Kaufmann. Citado na pág. 5

Lavalle(2006) S. M. Lavalle. Planning Algorithms. Cambridge University Press. Citado na pág. 17

Littman(1994) M. L. Littman. Markov games as a framework for multi-agent reinforcement learning. Em In Proceedings of the Eleventh International Conference on Machine Learning, páginas 157-163. Morgan Kaufmann. Citado na pág. 5, 17, 20

Littman(1996) M. L. Littman. Algorithms for sequential decision making. Tese de Doutorado, Department of Computer Science - Brown University, Providence, Rhode Island. Citado na pág. 3, 7, 16, 17, 18

McMahan et al.(2005) H. Brendan McMahan, Maxim Likhachev e Geoffrey J. Gordon. Bounded real-time dynamic programming: RTDP with monotone upper bounds and performance guarantees. Em ICML'05: Proceedings of the 22nd international conference on Machine learning, páginas 569-576, New York, NY, USA. ACM. Citado na pág. 1, 2, 10

Parthasarathy e Raghavan(1971) T. Parthasarathy e T. E. S. Raghavan. Some topics in two-person games. American Elsevier Pub. Co., New York. Citado na pág. 3

Pereira e de Barros(2007) S. L. Pereira e L. N. de Barros. Planejamento baseado em processos de decisão markovianos. Relatório técnico, Instituto de Matemática e Estatística. Citado na pág. 7, 10, 23 
Puterman(1987) M. L. Puterman. Dynamic programming. Em R.A. Meyers, editor, Encyclopedia of Physical Science and Technology, páginas (4)438-463. Academic Press. Citado na pág. 8, 9

Puterman(1994) M. L. Puterman. Markov Decision Processes. John Wiley and Sons, New York. Citado na pág. $1,2,5,8,10$

Quaeghebeur e de Cooman(2009) E. Quaeghebeur e G. de Cooman. Learning in games using the imprecise Dirichlet model. Em Elsevier Science Inc., editor, Int. J. Approx. Reasoning, páginas (50)243-256. New York, NY, USA. Citado na pág. 5

Russell e Norvig(2010) S. J. Russell e P. Norvig. Artificial Intelligence - A modern Approach. Prentice-Hall, New Jersey. Citado na pág. 1, 61

Satia e Lave Jr.(1973) J. K. Satia e R. E. Lave Jr. Markovian decision processes with uncertain transition probabilities. Operations Research, 21:728-740. Citado na pág. 2, 5, 33

Shapley(1953) L. S. Shapley. Stochastic games. National Academy of Sciences, 39:327-332. Citado na pág. 5, 17,18

Trevizan(2006) F. W. Trevizan. Um modelo unificado para planejamento sob incerteza. Dissertação de Mestrado, Instituto de Matemática e Estatística - USP, São Paulo, SP. Citado na pág. 1, 12, 13, 15

Trevizan e Veloso(2012) F. W. Trevizan e M. M. Veloso. Short-sighted stochastic shortest path problems. Em Lee McCluskey, Brian Williams, José Reinaldo Silva e Blai Bonet, editors, ICAPS. AAAI. Citado na pág. 10

Trevizan et al.(2007) F. W. Trevizan, F. G. Cozman e L. N. de Barros. Planning under risk and knightian uncertainty. Em Proceedings of the 20th international joint conference on Artifical intelligence, IJCAI'07, páginas 2023-2028, San Francisco, CA, USA. Morgan Kaufmann Publishers Inc. Citado na pág. 10, 13, 15

Utkin e Augustin(2005) L. V. Utkin e Th. Augustin. Powerful algorithms for decision making under partial prior information and general ambiguity attitudes. Em ISIPTA, páginas 349-358. Pittsburgh, Pennsylvania. Citado na pág. 46

Walley(1991) P. Walley. Statistical Reasoning with Imprecise Probabilities. Chapman and Hall, London. Citado na pág. 14, 28, 29

White III e Eldeib(1994) C. C. White III e H. K. Eldeib. Markov decision processes with imprecise transition probabilities. Em Operations Research, páginas (42)739-749. Citado na pág. 5, 33 